\title{
RANDOM CONDUCTANCE MODELS WITH STABLE-LIKE JUMPS: QUENCHED INVARIANCE PRINCIPLE
}

\author{
XIN CHEN TAKASHI KUMAGAI JIAN WANG
}

\begin{abstract}
We study the quenched invariance principle for random conductance models with long range jumps on $\mathbb{Z}^{d}$, where the transition probability from $x$ to $y$ is, on average, comparable to $|x-y|^{-(d+\alpha)}$ with $\alpha \in(0,2)$ but is allowed to be degenerate. Under some moment conditions on the conductance, we prove that the scaling limit of the Markov process is a symmetric $\alpha$-stable Lévy process on $\mathbb{R}^{d}$. The well-known corrector method in homogenization theory does not seem to work in this setting. Instead, we utilize probabilistic potential theory for the corresponding jump processes. Two essential ingredients of our proof are the tightness estimate and the Hölder regularity of caloric functions for non-elliptic $\alpha$-stable-like processes on graphs. Our method is robust enough to apply not only for $\mathbb{Z}^{d}$ but also for more general graphs whose scaling limits are nice metric measure spaces.
\end{abstract}

Keywords: random conductance model; long range jump; stable-like process; quenched invariance principle

MSC 2010: 60G51; 60G52; 60J25; 60J75.

\section{Introduction And Main Results}

Over the last decade, significant progress has been made concerning the quenched invariance principle on random conductance models. A typical and important example is random walk on the infinite cluster of supercritical bond percolation on $\mathbb{Z}^{d}$. It is shown that the scaling limit of the random walk is a (constant time change of) Brownian motion on $\mathbb{R}^{d}$ in the quenched sense, namely almost surely with respect to the randomness of the media. See $[2,9,14,17,21,35,36,39]$ for related progress on this subject and $[16,34]$ for overall introduction on this area and related topics. Besides i.i.d. nearest neighbor random conductance models, recently there have been great developments on the scaling limit of short range random conductance models on stationary ergodic media (or the media with suitable correlation conditions), see [3, 4, 5, 18, 30, 38] for more details. Here, short range means only finite number of conductances are directly connected to each vertex.

Unlike the short range case, there are only a few results concerning quenched invariance principle for long range random conductance models due to their fundamental technical difficulties. There is a beautiful paper by Crawford and Sly [28] that obtains the quenched invariance principle for random walk on the long range percolation cluster to an isotropic $\alpha$-stable Lévy process in the range $0<\alpha<1$. While [28] proves the invariance principle for a very singular object like the long range percolation, the arguments heavily rely on the special properties (see for instance [13, 15, 27] for related discussions) of the long range percolation and cannot be easily generalized to the setting of general (long range) random conductance models.

In this paper, we will discuss the quenched invariance principle on long range random conductance models. In particular, we consider the case where the conductance between $x$ and $y$ is, on average, comparable to $|x-y|^{-(d+\alpha)}$ with $\alpha \in(0,2)$ but is allowed to be degenerate. In this setting, there is a significant difficulty in applying classical techniques of homogenization for nearest neighbor random walk (in random environment) due to the existence of long range conductances. To emphasize the novelty of our paper, we first make some remarks. Some more details and technical difficulties of our methods are further discussed at the end of the introduction.

X. Chen: Department of Mathematics, Shanghai Jiao Tong University, 200240 Shanghai, P.R. China. chenxin217@sjtu.edu.cn.

T. Kumagai: Research Institute for Mathematical Sciences, Kyoto University, Kyoto 606-8502, Japan. kumagai@kurims.kyoto-u.ac.jp.

J. Wang: College of Mathematics and Informatics \& Fujian Key Laboratory of Mathematical Analysis and Applications, Fujian Normal University, 350007 Fuzhou, P.R. China. jianwang@fjnu.edu.cn. 
(i) The well known harmonic decomposition method (also called the corrector method in the literature) has been widely used for the nearest neighbor random walk in random media, see $[2,3,4,5,9,14,18,39]$. Because of the lack of $L^{2}$ integrability, such method does not work (at least in a straightforward way) for our long range model here.

(ii) Due to singularity in the infinite cluster of long range percolation, [28] established the quenched invariance principle of the associated random walk in the sense of weak convergence on $L^{q}$ (not the Skorohod topology) and only for the case $0<\alpha<1$. In the present paper, we can justify quenched invariance principle of our model under the Skorohod topology for all $\alpha \in(0,2)$. (To be fair, the long range percolation is "more singular", and it is not included in our conductance model.) Moreover, compared with [23], we can prove the quenched invariance principle for the process with fixed initial point, see e.g. Remark 4.6 below.

(iii) Our approach is to utilize recently developed de Giorgi-Nash-Moser theory for jump processes (see for instance [7, 24, 25, 26]). While detailed heat kernel estimates and Harnack inequalities are established for uniformly elliptic $\alpha$-stable-like processes, the arguments rely on pointwise estimates of the jumping density (conductance in this setting), which cannot hold in our setting unless we assume uniform ellipticity of conductance. Furthermore, as will be shown in the accompanied paper [20], Harnack inequalities do not hold (even for large enough balls) in general on long range random conductance models. For these reasons, highly non-trivial modifications are required to work on the present random conductance setting. Roughly speaking, in this paper we are concerned with the long range conductance model with large scale summability conditions on the conductance, which can be viewed as a counterpart of the so-called "good ball condition" in $[6,8]$ to the non-local setting. We believe that our methods are rather robust and could be fundamental tools in exploring scaling limits of random walks on long range random media.

(iv) The advantage of our methods is that they do not use translation invariance of the original graph (we do not use the idea of "the environment viewed from the particle"); hence they are applicable not only for $\mathbb{Z}^{d}$ but also for more general graphs whose scaling limits are nice metric measure spaces. Even in the setting of $\mathbb{Z}^{d}$, our results can be applied to the case that the conductance is independent but possibly degenerate and not necessarily identically distributed; that is, our results are efficient for some long range random walks on degenerate non-ergodic media. The disadvantage is, since we use the Borel-Cantelli lemma to deduce quenched estimates, the arguments require "strong mixing properties" of the random conductance (see (5.4)-(5.10) below). Hence our method cannot be generalized to general stationary ergodic case on $\mathbb{Z}^{d}$.

To illustrate our contribution, we present the statement about the quenched invariance principle on a half/quarter space $F:=\mathbb{R}_{+}^{d_{1}} \times \mathbb{R}^{d_{2}}$ where $d_{1}, d_{2} \in \mathbb{N} \cup\{0\}$. (This is the simplest example of state spaces that is not translation invariant when $d_{1} \neq 0$.) The readers may refer to Sections 4 and 5 for general results. Let $\mathbb{L}:=\mathbb{Z}_{+}^{d_{1}} \times \mathbb{Z}^{d_{2}}$. Consider a Markov generator

$$
L_{\mathbb{L}}^{\omega} f(x)=\sum_{y \in \mathbb{L}}(f(y)-f(x)) \frac{w_{x, y}(\omega)}{|x-y|^{d+\alpha}}, \quad x \in \mathbb{L},
$$

where $d=d_{1}+d_{2}, \alpha \in(0,2)$ and $\left\{w_{x, y}(\omega): x, y \in \mathbb{L}\right\}$ is a sequence of random variables such that $w_{x, y}(\omega)=w_{y, x}(\omega) \geqslant 0$ for all $x \neq y$. We use the convention that $w_{x, x}(\omega)=w_{x, x}^{-1}(\omega)=0$ for all $x \in \mathbb{L}$. Let $\left(X_{t}^{\omega}\right)_{t \geqslant 0}$ be the corresponding Markov process. For every $n \geqslant 1$ and $\omega \in \Omega$, we define a process $X^{(n), \omega}$ on $V_{n}=n^{-1} \mathbb{L}$ by $X_{t}^{(n), \omega}:=n^{-1} X_{n^{\alpha} t}^{\omega}$ for any $t \geqslant 0$. Let $\mathbb{P}_{x}^{(n), \omega}$ be the law of $X^{(n), \omega}$ with initial point $x \in V_{n}$. Let $Y:=\left(\left(Y_{t}\right)_{t \geqslant 0},\left(\mathbb{P}_{x}^{Y}\right)_{x \in F}\right)$ be a $F$-valued strong Markov process. We say that the quenched invariance principle holds for $X^{\omega}$. with limit process being $Y$, if for any $\left\{x_{n} \in V_{n}: n \geqslant 1\right\}$ such that $\lim _{n \rightarrow \infty} x_{n}=x$ for some $x \in F$, it holds that for P-a.s. $\omega \in \Omega$ and every $T>0, \mathbb{P}_{x_{n}}^{(n), \omega}$ converges weakly to $\mathbb{P}_{x}^{Y}$ on the space of all probability measures on $\mathscr{D}([0, T] ; F)$, the collection of càdlàg $F$-valued functions on $[0, T]$ equipped with the Skorohod topology.

Theorem 1.1. Let $d>4-2 \alpha$, and $E=\{(x, y): x, y \in \mathbb{L}\}$ be the collection of all unordered pairs on $\mathbb{L}$. Suppose that $\left\{w_{x, y}:(x, y) \in E\right\}$ is a sequence of non-negative independent random variables 
such that $\mathbb{E} w_{x, y}=1$ for all $x, y \in \mathbb{L}$,

$$
\sup _{x, y \in \mathbb{L}, x \neq y} \mathbb{P}\left(w_{x, y}=0\right)<1 / 2
$$

and

$$
\sup _{x, y \in \mathbb{L}} \mathbb{E}\left[w_{x, y}^{2 p}\right]<\infty, \quad \sup _{x, y \in \mathbb{L}} \mathbb{E}\left[w_{x, y}^{-2 q} \mathbb{1}_{\left\{w_{x, y}>0\right\}}\right]<\infty
$$

with

$$
p>\max \{(d+2) / d,(d+1) /(2(2-\alpha))\}, \quad q>(d+2) / d .
$$

Then the quenched invariance principle holds for $X^{\omega}$. with the limit process being a reflected symmetric $\alpha$-stable process $Y$ on $F$ with jumping measure $|z|^{-d-\alpha} d z$.

Remark 1.2. (i) When $\alpha \in(0,1)$, the conclusion of Theorem 1.1 still holds true for $d>2-2 \alpha$, if $p>\max \{(d+2) / d,(d+1) /(2(1-\alpha))\}$ and $q>(d+2) / d$. See Proposition 5.6 for details.

(ii) The probability $1 / 2$ in (1.2) is far from optimal. In fact, the critical probability $p_{c}$ allowing the conductances to be degenerate heavily depends on large scale properties of the long-range percolation cluster associated with the random conductance model in our paper, which are different from these of the nearest neighbor percolation cluster (see e.g. $[2,6,14,21,35,39]$ ) or these of the long-range percolation cluster investigated in $[13,15,27,28]$. We do not know the exact value of $p_{c}$, and even whether $p_{c}=1$ or $p_{c}<1$.

(iii) We note that the integrability condition (1.4) is far from optimal too, and we also do not even know what could be the optimal integrability condition. Furthermore, by tracking the proofs of Lemma 2.2 and Proposition 5.6 below, the negative moment condition $\sup _{x, y \in \mathbb{L}} \mathbb{E}\left[w_{x, y}^{-2 q} \mathbb{1}_{\left\{w_{x, y}>0\right\}}\right]<\infty$ in (1.3) is only required to guarantee the local Poincaré inequality (2.11). For the i.i.d nearest neighbor percolation cluster, such kind of negative moment condition can be removed by the domination behavior of the percolation cluster and time change arguments, see e.g. [2, 35]. However, as we mentioned above, since properties of the nearest neighbor percolation cluster are quite different from those of long-range percolation cluster associated with the random conductance model in our paper, the arguments in $[2,35]$ do not work for the present setting. By now we do not know whether this negative moment condition is essentially necessary.

Here is one simple example that satisfies (1.2) and (1.3): for each distinct $x, y \in \mathbb{L}$,

$$
\begin{gathered}
\mathbb{P}\left(w_{x, y}=|x-y|^{\varepsilon}\right)=\left(3|x-y|^{2 p \varepsilon}\right)^{-1}, \quad \mathbb{P}\left(w_{x, y}=|x-y|^{-\delta}\right)=\left(3|x-y|^{2 q \delta}\right)^{-1}, \\
\mathbb{P}\left(w_{x, y}=0\right)=3^{-1}, \quad \mathbb{P}\left(w_{x, y}=g(x, y)\right)=1-\left(3|x-y|^{2 p \varepsilon}\right)^{-1}-\left(3|x-y|^{2 q \delta}\right)^{-1}-3^{-1},
\end{gathered}
$$

where $\varepsilon, \delta, p, q>0$ so that (1.4) is satisfied, and $g(x, y)$ are chosen so that $\mathbb{E} w_{x, y}=1$. (It is easy to see that $c^{-1} \leqslant g(x, y) \leqslant c$ for some constant $c \geqslant 1$.)

At the end of the introduction, let us briefly discuss technical difficulties and the ideas of the proof. There are two essential ingredients in our proof; namely the tightness estimate and the Hölder regularity of caloric functions for non-elliptic $\alpha$-stable-like processes on graphs. In order to obtain the former estimate, we first split small jumps and big jumps, which is a standard approach for jump processes, and then change the conductance to the averaged one outside a ball (we call it the localization method). By this technique and the on-diagonal heat kernel upper bound (Proposition 2.1), we can apply the so-called Bass-Nash method to control the mean displacement of the process (Proposition 2.4). The tightness estimate (Theorem 3.3) is established by comparing the original process, truncated process and the localized process. We note that when $0<\alpha<1$, tightness can be proved in a much simpler way using martingale arguments (Proposition 3.5). The key ingredient for the Hölder regularity of caloric functions (Theorem 3.6) is to deduce the Krylov-type estimate (Proposition 3.8) that controls the hitting probability to a large set before exiting some parabolic cylinder. Once these estimates are established, we use the arguments in [23] to deduce generalized Mosco convergence, and then obtain the weak convergence (Theorem 4.5). 


\section{TRUnCATED $\alpha$-STABle-Like PROCESSES ON GRAPHS}

In this and the next sections, we fix graphs and discuss $\alpha$-stable-like processes on them. Hence we do not consider randomness of the environment. With a slight abuse of notation, we still use $w_{x, y}$ as the deterministic version. Let $G=\left(V, E_{V}\right)$ be a locally finite and connected graph, where $V$ is the set of vertices, and $E_{V}$ the set of edges. For any $x \neq y \in V$, we write $\rho(x, y)$ for the graph distance, i.e., $\rho(x, y)$ is the length of the shortest path (that is, a sequence $x_{0}=x, x_{1}, \cdots, x_{l}=y$ such that $\left(x_{i}, x_{i+1}\right) \in E_{V}$ for all $\left.0 \leqslant i \leqslant l-1\right)$ joining $x$ and $y$. Set $\rho(x, x)=0$ for all $x \in V$. We let $B(x, r)=\{y \in V: \rho(x, y) \leqslant r\}$ denote the ball in the graph metric with center $x \in V$ and radius $r>0$. Let $\mu$ be a measure on $V$ such that $\mu_{x}:=\mu(\{x\})$ satisfies for some constant $c_{M} \geqslant 1$ that

$$
c_{M}^{-1} \leqslant \mu_{x} \leqslant c_{M}, \quad x \in V .
$$

For each $p \in[1, \infty)$, let $L^{p}(V ; \mu)=\left\{f \in \mathbb{R}^{V}: \sum_{x \in V}|f(x)|^{p} \mu_{x}<\infty\right\}$, and denote by $\|f\|_{p}$ the $L^{p}$ norm of $f$ with respect to $\mu$. Let $L^{\infty}(V ; \mu)$ be the space of bounded measurable functions on $V$, and let $\|f\|_{\infty}$ be the $L^{\infty}$ norm of $f$. We assume that $(G, \mu)$ satisfies the $d$-set condition with $d>0$, i.e., there exist $r_{G} \in[1, \infty]$ and $c_{G} \geqslant 1$ such that

$$
c_{G}^{-1} r^{d} \leqslant \mu(B(x, r)) \leqslant c_{G} r^{d}, \quad x \in V, 1 \leqslant r<r_{G} .
$$

For example, if $V$ is a subset of $\mathbb{Z}^{d}$, then we can take $r_{G}=1+\operatorname{diam} V$, where $\operatorname{diam} V$ denotes the diameter of $V$. In particular, when $V$ is bounded, $r_{G}<+\infty-$ see Section 5.2.3. Throughout the paper, all the constants appearing in the statements of lemmas, propositions and theorems are independent of $r_{G}$. We will also not stress the dependence on $c_{G}$ and $c_{M}$ above for these constants.

We consider the operator $L f(x)=\sum_{z \in V}(f(z)-f(x)) \frac{w_{x, z}}{\rho(x, z)^{d+\alpha}} \mu_{z}$ and the quadratic form

$$
D(f, f)=\frac{1}{2} \sum_{x, y \in V}(f(x)-f(y))^{2} \frac{w_{x, y}}{\rho(x, y)^{d+\alpha}} \mu_{x} \mu_{y}, \quad f \in \mathscr{F}=\left\{f \in L^{2}(V ; \mu): D(f, f)<\infty\right\},
$$

where $\alpha \in(0,2)$ and $\left\{w_{x, y}: x, y \in V\right\}$ is a sequence such that $w_{x, x}=0$ for all $x \in V, w_{x, y} \geqslant 0$ and $w_{x, y}=w_{y, x}$ for all $x \neq y$, and

$$
\sum_{y \in V} \frac{w_{x, y}}{\rho(x, y)^{d+\alpha}} \mu_{y}<\infty, \quad x \in V
$$

Here by convention we set $0 / 0=0$. According to (the first statement in) $[23$, Theorem 3.2], $(D, \mathscr{F})$ is a regular symmetric Dirichlet form on $L^{2}(V ; \mu)$. Let $X:=\left(X_{t}\right)_{t \geqslant 0}$ be the symmetric Hunt process associated with $(D, \mathscr{F})$. Set $C_{x, y}:=w_{x, y} / \rho(x, y)^{d+\alpha}$. Under $\mathbb{P}^{x}, X_{0}=x$, and the process $X$ waits for an exponentially distributed random time of parameter $C_{x}:=\sum_{y \in V} C_{x, y} \mu_{y}$ and jumps to point $y \in V$ with probability $C_{x, y} \mu_{y} / C_{x}$; this procedure is then iterated choosing independent hopping times. Such a Markov process $X$ is called a variable speed $\alpha$-stable-like random walk on $V$ - see Section 5.2 .5 concerning a constant speed $\alpha$-stable-like random walk. We write $p(t, x, y)$ for the heat kernel of $X$ on $V$; that is, the transition density of the process $X$ with respect to $\mu$ which is defined by $p(t, x, y)=\mu_{y}^{-1} \mathbb{P}^{x}\left(X_{t}=y\right)$.

The goal of this section is to study moment estimates for truncated $\alpha$-stable-like processes on graphs, which are crucial to obtain probability estimates for the exit time of the (original) process. For this, we first consider on-diagonal upper bounds for heat kernels of the truncated process in Subsection 2.1. Then, by adopting the localization method and using the idea of Bass-Nash, in Subsection 2.2 we establish moment estimates for the truncation of the localized $\alpha$-stable-like process on graphs.

2.1. On-diagonal upper bounds for heat kernel. In this subsection, we are concerned with the truncated Dirichlet form corresponding to $(D, \mathscr{F})$. For fixed $1 \leqslant \delta<r_{G}$, define the operator $L^{\delta} f(x)=\sum_{z \in V: \rho(z, x) \leqslant \delta}(f(z)-f(x)) \frac{w_{z, x}}{\rho(z, x)^{d+\alpha}} \mu_{z}$. Then, the associated bilinear form is given by

$$
D^{\delta}(f, f)=\frac{1}{2} \sum_{x, y \in V: \rho(x, y) \leqslant \delta}(f(x)-f(y))^{2} \frac{w_{x, y}}{\rho(x, y)^{d+\alpha}} \mu_{x} \mu_{y} .
$$


Throughout this part, we always assume that

$$
C_{V, \delta}:=\sup _{x \in V} \sum_{y \in V: \rho(x, y)>\delta} \frac{w_{x, y}}{\rho(x, y)^{d+\alpha}} \mu_{y}<\infty .
$$

By (2.4) and the symmetry of $w_{x, y}$, we can easily see that for all $f \in \mathscr{F}$,

$$
D^{\delta}(f, f) \leqslant D(f, f) \leqslant D^{\delta}(f, f)+2 \sum_{x \in V} f(x)^{2} \mu_{x} \sum_{y \in V: \rho(y, x)>\delta} \frac{w_{x, y}}{\rho(x, y)^{d+\alpha}} \mu_{y} \leqslant D^{\delta}(f, f)+2 C_{V, \delta}\|f\|_{2}^{2} .
$$

Consequently, $\left(D^{\delta}, \mathscr{F}\right)$ is also a regular and symmetric Dirichlet form on $L^{2}(V ; \mu)$. Denote by $X^{\delta}:=$ $\left(\left(X_{t}^{\delta}\right)_{t \geqslant 0},\left(\mathbb{P}_{x}\right)_{x \in V}\right)$ the associated Hunt process, which is called the truncated process associated with $X$ in the literature.

In the following, we denote by $p^{\delta}(t, x, y)$ the heat kernel of $X^{\delta}$. Given a sequence of $w:=\left\{w_{x, y}\right.$ : $x, y \in V\}$, we set $B^{w}(x, r):=\left\{z \in B(x, r): w_{x, z}>0\right\}$ for every $x \in V$ and $r \geqslant 1$. The main statement in this part is as follows.

Proposition 2.1. Suppose that (2.4) holds for some $\delta>0$, and that there exist constants $\theta \in(0,1)$ and $C_{1}, C_{2}>0$ such that for every $\delta^{\theta} \leqslant r \leqslant \delta$,

$$
\begin{aligned}
& \sup _{x \in V} \sum_{y \in B^{w}(x, r)} w_{x, y}^{-1} \leqslant C_{1} r^{d}, \\
& \inf _{x \in V} \mu\left(B^{w}(x, r)\right) \geqslant C_{2} r^{d}
\end{aligned}
$$

and

$$
\sup _{x \in V} \sum_{y \in V: \rho(y, x) \leqslant r} \frac{w_{x, y}}{\rho(x, y)^{d+\alpha-2}} \leqslant C_{1} r^{2-\alpha} .
$$

Then, for each $\theta^{\prime} \in(\theta, 1)$, there are constants $\delta_{0}>0$ (depending only on $\theta^{\prime}, \theta$ ) and $C_{3}>0$ such that when the constant $\delta>0$ above satisfies $\delta_{0} \leqslant \delta<r_{G}$, the following estimate holds

$$
p^{\delta}(t, x, y) \leqslant C_{3} t^{-d / \alpha}, \quad \forall 2 \delta^{\theta^{\prime} \alpha} \leqslant t \leqslant \delta^{\alpha} \text { and } x, y \in V .
$$

In order to get on-diagonal upper bounds for the heat kernel of the truncated process $X^{\delta}$, we need the following scaled Poincaré-type inequality. We note that the inequality (2.11) below is different from the standard Poincaré inequality since here the term $(f)_{B\left(x, r_{0}\right)}$ (for the standard Poincaré inequality) is replaced by $(f)_{B^{w}\left(z, r_{0}\right)}$, which will be adopted to deal with the case that $w_{x, y}$ is degenerate.

Lemma 2.2. Suppose that there exist constants $C_{1}, C_{2}>0$ and $1 \leqslant r_{0}<r_{G}$ such that

$$
\sup _{x \in V} \sum_{y \in B^{w}\left(x, r_{0}\right)} w_{x, y}^{-1} \leqslant C_{1} r_{0}^{d}
$$

and

$$
\inf _{x \in V} \mu\left(B^{w}\left(x, r_{0}\right)\right) \geqslant C_{2} r_{0}^{d}
$$

Then there is a constant $C_{3}>0$, independent of $r_{0}$, such that for all $x \in V$ and measurable function $f$ on $V$,

$$
\sum_{z \in B\left(x, r_{0}\right)}\left(f(z)-(f)_{B^{w}\left(z, r_{0}\right)}\right)^{2} \mu_{z} \leqslant C_{3} r_{0}^{\alpha} \sum_{y, z \in V: z \in B\left(x, r_{0}\right), y \in B\left(z, r_{0}\right)}(f(z)-f(y))^{2} \frac{w_{z, y}}{\rho(z, y)^{d+\alpha}} \mu_{z} \mu_{y},
$$

where for $A \subset V,(f)_{A}:=\mu(A)^{-1} \sum_{z \in A} f(x) \mu_{z}$.

Proof. For every $x \in V$ and measurable function $f$ on $V$, we have

$$
\begin{aligned}
& \sum_{z \in B\left(x, r_{0}\right)}\left(f(z)-(f)_{B^{w}\left(z, r_{0}\right)}\right)^{2} \mu_{z}=\sum_{z \in B\left(x, r_{0}\right)}\left(\frac{1}{\mu\left(B^{w}\left(z, r_{0}\right)\right)} \sum_{y \in B^{w}\left(z, r_{0}\right)}(f(z)-f(y)) \mu_{y}\right)^{2} \mu_{z} \\
\leqslant & \frac{c_{1}}{r_{0}^{2 d}} \sum_{z \in B\left(x, r_{0}\right)}\left[\left(\sum_{y \in B^{w}\left(z, r_{0}\right)}(f(z)-f(y))^{2} \frac{w_{z, y}}{\rho(z, y)^{d+\alpha}}\right)\left(\sum_{y \in B^{w}\left(z, r_{0}\right)} w_{z, y}^{-1} \rho(z, y)^{d+\alpha}\right)\right]
\end{aligned}
$$




$$
\begin{aligned}
& \leqslant c_{2} r_{0}^{-d+\alpha}\left(\sup _{z \in V} \sum_{y \in B^{w}\left(z, r_{0}\right)} w_{z, y}^{-1}\right)\left(\sum_{y, z \in V: z \in B\left(x, r_{0}\right), y \in B\left(z, r_{0}\right)}(f(z)-f(y))^{2} \frac{w_{z, y}}{\rho(z, y)^{d+\alpha}}\right) \\
& \leqslant c_{3} r_{0}^{\alpha} \sum_{y, z \in V: z \in B\left(x, r_{0}\right), y \in B\left(z, r_{0}\right)}(f(z)-f(y))^{2} \frac{w_{z, y}}{\rho(z, y)^{d+\alpha}} \mu_{z} \mu_{y},
\end{aligned}
$$

where the first inequality follows from (2.1), (2.10) and the Cauchy-Schwarz inequality, in the second inequality we have used the fact that $\rho(z, y) \leqslant r_{0}$ for every $y \in B^{w}\left(z, r_{0}\right)$, and the third inequality is due to (2.1) and (2.9). This proves (2.11).

We are now in a position to present the

Proof of Proposition 2.1. Without mention, throughout the proof constant $c_{i}$ will be independent of $\delta, t, x, y$ and $r_{G}$. Since, by the Cauchy-Schwarz inequality,

$$
p^{\delta}(t, x, y) \leqslant p^{\delta}(t, x, x)^{1 / 2} p^{\delta}(t, y, y)^{1 / 2}
$$

for any $t>0$ and $x, y \in V$, it suffices to verify (2.8) for the case that $x=y$. The proof is split into three steps.

Step (1): We first note that under (2.4) and (2.7), $\sup _{x \in V} \sum_{y \in V} \frac{w_{x, y}}{\rho(x, y)^{d+\alpha}} \mu_{y}<\infty$. This along with (the second statement in) [23, Theorem 3.2] yields that the process $X^{\delta}$ is conservative. By [29, Proposition 5 and Theorem 8], we have the following upper bound for $p^{\delta}(t, x, y)$ :

$$
p^{\delta}\left(t, x_{1}, x_{2}\right) \leqslant \mu_{x_{1}}^{-1 / 2} \mu_{x_{2}}^{-1 / 2} \inf _{\phi \in L^{\infty}(V ; \mu)} \exp \left(\phi\left(x_{1}\right)-\phi\left(x_{2}\right)+b(\phi) t\right)
$$

for all $t>0$ and $x_{1}, x_{2} \in V$, where

$$
b(\phi):=\frac{1}{2} \sup _{x \in V} \sum_{y \in V: \rho(y, x) \leqslant \delta} \frac{w_{x, y}}{\rho(x, y)^{d+\alpha}}\left(e^{\phi(y)-\phi(x)}+e^{\phi(x)-\phi(y)}-2\right) \mu_{y} .
$$

For fixed $x_{1}, x_{2} \in V$, taking $\phi(x)=\rho\left(x, x_{1}\right) \wedge \rho\left(x_{1}, x_{2}\right)$ for any $x \in V$, we get that

$$
\begin{aligned}
b(\phi) & \leqslant \frac{1}{2} \sup _{x \in V} \sum_{y \in V: \rho(y, x) \leqslant \delta} \frac{w_{x, y}}{\rho(x, y)^{d+\alpha}}\left(e^{\rho(x, y)}+e^{-\rho(x, y)}-2\right) \mu_{y} \\
& \leqslant \frac{1}{2} \sup _{x \in V} \sum_{y \in V: \rho(y, x) \leqslant \delta} \frac{w_{x, y}}{\rho(y, x)^{d+\alpha}} \rho(x, y)^{2} e^{\rho(x, y)} \mu_{y} \\
& \leqslant \frac{1}{2} e^{\delta} \sup _{x \in V} \sum_{y \in V: \rho(y, x) \leqslant \delta} \frac{w_{x, y}}{\rho(x, y)^{d+\alpha-2}} \mu_{y} \leqslant c_{1} e^{\delta} \delta^{2-\alpha} \leqslant 2 c_{1} e^{2 \delta},
\end{aligned}
$$

where in the first inequality above we have used the facts that $s \mapsto e^{s}+e^{-s}$ is increasing on $[0, \infty)$ and $|\phi(x)-\phi(y)| \leqslant \rho(x, y)$ for all $x, y \in V$, the second inequality is due to the fact that $e^{s}+e^{-s}-2 \leqslant s^{2} e^{s}$ for all $s \geqslant 0$, and the fourth inequality follows from (2.7). Combining this with (2.12), we arrive at the statement that for all $t>0$ and $x_{1}, x_{2} \in V$,

$$
p^{\delta}\left(t, x_{1}, x_{2}\right) \leqslant c_{M} \exp \left(-\rho\left(x_{1}, x_{2}\right)+2 c_{1} e^{2 \delta} t\right) .
$$

Furthermore, it follows from the symmetry of $w_{x, y}$, the fact that $p^{\delta}(t, x, y) \mu_{y} \leqslant 1$ for all $t>0$ and $x, y \in V,(2.7)$ and (2.13) that for every $x \in V$,

$$
\begin{aligned}
& \sum_{z, v \in V: \rho(z, v) \leqslant \delta}\left(p^{\delta}(t, x, z)-p^{\delta}(t, x, v)\right)^{2} \frac{w_{z, v}}{\rho(z, v)^{d+\alpha}} \mu_{z} \mu_{v} \\
& \leqslant \sum_{z, v \in V: \rho(z, v) \leqslant \delta}\left(p^{\delta}(t, x, z)+p^{\delta}(t, x, v)\right)^{2} \frac{w_{z, v}}{\rho(z, v)^{d+\alpha}} \mu_{z} \mu_{v} \\
& \leqslant 4 c_{M} \sum_{z \in V} p^{\delta}(t, x, z)\left(\sup _{z \in V} \sum_{v \in V: \rho(v, z) \leqslant \delta} \frac{w_{z, v}}{\rho(z, v)^{d+\alpha}}\right) \\
& \leqslant 4 c_{M} \sum_{z \in V} p^{\delta}(t, x, z)\left(\sup _{z \in V} \sum_{v \in V: \rho(z, v) \leqslant \delta} \frac{w_{z, v}}{\rho(z, v)^{d+\alpha-2}}\right) \leqslant c_{2}(\delta, t) \sum_{z \in V} \exp (-\rho(z, x))<\infty,
\end{aligned}
$$


where in the last inequality we used the fact that

$$
\sum_{z \in V} \exp (-\rho(z, x)) \leqslant c_{M} \sum_{r=0}^{\infty} \sum_{z \in V: \rho(x, z)=r} e^{-r} \mu_{z} \leqslant c_{M} \sum_{r=0}^{\infty} \mu(B(x, r)) e^{-r} \leqslant c_{M} c_{G} \sum_{r=1}^{\infty} r^{d} e^{-r}<\infty .
$$

Therefore, according to the Fubini theorem and (2.13), for every $x \in V$,

$$
\sum_{z \in V} L^{\delta} p^{\delta}(t, x, \cdot)(z) p^{\delta}(t, x, z) \mu_{z}=-\frac{1}{2} \sum_{z, v \in V: \rho(z, v) \leqslant \delta}\left(p^{\delta}(t, x, z)-p^{\delta}(t, x, v)\right)^{2} \frac{w_{z, v}}{\rho(z, v)^{d+\alpha}} \mu_{z} \mu_{v} .
$$

Step (2): Below we fix $x \in V$. Let $f_{t}(z)=p^{\delta}(t, x, z)$ and $\psi(t)=p^{\delta}(2 t, x, x)$ for all $z \in V$ and $t \geqslant 0$. Then, $\psi(t)=\sum_{z \in V} f_{t}(z)^{2} \mu_{z}$, and, by $(2.14)$,

$$
\psi^{\prime}(t)=2 \sum_{z \in V} \frac{d f_{t}(z)}{d t} f_{t}(z) \mu_{z}=2 \sum_{z \in V} L^{\delta} f_{t}(z) f_{t}(z) \mu_{z}=-\sum_{z, y \in V: \rho(z, y) \leqslant \delta}\left(f_{t}(z)-f_{t}(y)\right)^{2} \frac{w_{z, y}}{\rho(z, y)^{d+\alpha}} \mu_{z} \mu_{y} .
$$

Let $\delta^{\theta} \leqslant r(t) \leqslant \delta$ and $R:=R(\delta) \geqslant 1$ be some constants to be determined later. Suppose that $B\left(x_{i}, r(t) / 2\right)(i=1, \cdots, m)$ is a maximal collection of disjoint balls with centers in $B(x, R)$. Set $B_{i}=B\left(x_{i}, r(t)\right)$ and $B_{i}^{*}=B\left(x_{i}, 2 r(t)\right)$. Then, $B(x, R) \subset \cup_{i=1}^{m} B_{i} \subset B(x, R+r(t)) \subset \cup_{i=1}^{m} B_{i}^{*}$. If $z \in B(x, R+r(t)) \cap B_{i}^{*}$ for some $1 \leqslant i \leqslant m$, then $B\left(x_{i}, r(t) / 2\right) \subset B(z, 3 r(t))$, and so

$$
c_{3} r(t)^{d} \geqslant \mu(B(z, 3 r(t))) \geqslant \sum_{i=1}^{m} \mathbb{1}_{\left\{z \in B_{i}^{*}\right\}} \mu\left(B\left(x_{i}, r(t) / 2\right)\right) \geqslant c_{4} r(t)^{d}\left|\left\{i: z \in B_{i}^{*}\right\}\right| .
$$

In the second inequality we used the fact that $B\left(x_{i}, r(t) / 2\right), i=1, \cdots, m$, are disjoint, and in the first and the last inequality we have used (2.2). Thus, every $z \in B(x, R+r(t))$ is in at most $c_{5}:=c_{3} / c_{4}$ of the balls $B_{i}^{*}$ (hence at most $c_{5}$ of the balls $B_{i}$ ). In particular,

$$
\sum_{i=1}^{m} \sum_{z \in B_{i}}=\sum_{i=1}^{m} \sum_{z \in B(x, R+r(t))} \mathbb{1}_{B_{i}}(z)=\sum_{z \in B(x, R+r(t))} \sum_{i=1}^{m} \mathbb{1}_{B_{i}}(z) \leqslant c_{5} \sum_{z \in B(x, R+r(t))} .
$$

According to (the proof of) Lemma 2.2, (2.5) and (2.6) imply that for every $\delta^{\theta} \leqslant r \leqslant \delta, x \in V$ and measurable function $f$ on $V$,

$$
\sum_{z \in B(x, r)}\left(f(z)-(f)_{B^{w}(z, r)}\right)^{2} \mu_{z} \leqslant c_{6} r^{\alpha} \sum_{z, y \in V: z \in B(x, r), y \in B(z, r)}(f(z)-f(y))^{2} \frac{w_{z, y}}{\rho(z, y)^{d+\alpha}} \mu_{z} \mu_{y} .
$$

Hence, noticing that $\delta^{\theta} \leqslant r(t) \leqslant \delta$,

$$
\begin{aligned}
& \sum_{z, y \in V: \rho(z, y) \leqslant \delta}\left(f_{t}(z)-f_{t}(y)\right)^{2} \frac{w_{z, y}}{\rho(z, y)^{d+\alpha}} \mu_{z} \mu_{y} \geqslant \frac{1}{c_{5}} \sum_{i=1}^{m} \sum_{z \in B_{i}} \sum_{y \in B(z, r(t))}\left(f_{t}(z)-f_{t}(y)\right)^{2} \frac{w_{z, y}}{\rho(z, y)^{d+\alpha}} \mu_{z} \mu_{y} \\
& \geqslant \frac{c_{7}}{r(t)^{\alpha}}\left[\sum_{i=1}^{m} \sum_{z \in B_{i}} f_{t}^{2}(z) \mu_{z}-2 \sum_{i=1}^{m} \sum_{z \in B_{i}} f_{t}(z)\left(f_{t}\right)_{B^{w}(z, r(t))} \mu_{z}\right]=: \frac{c_{7}}{r(t)^{\alpha}}\left(I_{1}-I_{2}\right),
\end{aligned}
$$

where in the second inequality we have used (2.16).

Furthermore, since $f_{t}(z) \mu_{z} \leqslant 1$ for all $z \in V$ and $t>0$, we have

$$
I_{1} \geqslant \sum_{z \in \cup_{i=1}^{m} B_{i}} f_{t}^{2}(z) \mu_{z} \geqslant \sum_{z \in B(x, R)} f_{t}^{2}(z) \mu_{z}=\psi(t)-\sum_{z \in V: \rho(z, x)>R} f_{t}^{2}(z) \mu_{z} \geqslant \psi(t)-\sum_{z \in V: \rho(z, x)>R} f_{t}(z) .
$$

So, by (2.13), we can choose $R:=R(\delta)=2 c_{1} e^{4 \delta}$ such that for all $\delta^{\theta \alpha} \leqslant t \leqslant \delta^{\alpha}$,

$$
\begin{aligned}
\sum_{z \in V: \rho(z, x)>R} f_{t}(z) & \leqslant \sum_{z \in V: \rho(z, x)>2 c_{1} e^{4 \delta}} \exp \left(-\rho(z, x)+2 c_{1} e^{2 \delta} \delta^{\alpha}\right) \\
& \leqslant c_{M} \sum_{z \in V: \rho(z, x)>2 c_{1} e^{4 \delta}} \exp (-\rho(z, x) / 2) \mu_{z} \\
& \leqslant c_{M} \sum_{r=2 c_{1} e^{4 \delta}}^{\infty} \mu(B(x, r)) e^{-r / 2} \leqslant c_{8} \delta^{-d} \leqslant c_{8} r(t)^{-d},
\end{aligned}
$$


where the last inequality follows from the fact that $r(t) \leqslant \delta$. On the other hand, due to (2.6) and the fact that $\sum_{z \in V} f_{t}(z) \mu_{z} \leqslant 1$ for all $t>0$,

$$
\sup _{z \in V}\left(f_{t}\right)_{B^{w}(z, r(t))} \leqslant \sup _{z \in V} \mu\left(B^{w}(z, r(t))\right)^{-1} \cdot \sum_{z \in V} f_{t}(z) \mu_{z} \leqslant C_{2}^{-1} r(t)^{-d} .
$$

This along with (2.15) yields that

$$
I_{2} \leqslant C_{2}^{-1} r(t)^{-d} \sum_{i=1}^{m} \sum_{z \in B_{i}} f_{t}(z) \mu_{z} \leqslant C_{2}^{-1} c_{5} r(t)^{-d} \sum_{z \in B(x, R+r(t))} f_{t}(z) \mu_{z} \leqslant C_{2}^{-1} c_{5} r(t)^{-d} .
$$

Therefore, combining all estimates above, we arrive at the statement that for every $\delta^{\theta} \leqslant r(t) \leqslant \delta$,

$$
\psi^{\prime}(t) \leqslant-c_{9} r(t)^{-\alpha}\left(\psi(t)-c_{10} r(t)^{-d}\right) .
$$

Step (3): For any $\theta^{\prime} \in(\theta, 1)$ and any $1 \leqslant \delta<r_{G}$ large enough, we claim that there exists $t_{0} \in\left[\delta^{\theta \alpha}, \delta^{\theta^{\prime} \alpha}\right]$ such that

$$
\left(\frac{1}{2 c_{10}} \psi\left(t_{0}\right)\right)^{-1 / d} \geqslant \delta^{\theta}
$$

Indeed, suppose that (2.18) does not hold. Then,

$$
\left(\frac{1}{2 c_{10}} \psi(t)\right)^{-1 / d}<\delta^{\theta}, \quad \forall \delta^{\theta \alpha} \leqslant t \leqslant \delta^{\theta^{\prime} \alpha},
$$

which means that $\psi(t) \geqslant 2 c_{10} \delta^{-d \theta}$ for all $\delta^{\theta \alpha} \leqslant t \leqslant \delta^{\theta^{\prime} \alpha}$. Hence, taking $r(t)=\delta^{\theta}$ in (2.17), we find that $\psi^{\prime}(t) \leqslant-2^{-1} c_{9} \delta^{-\theta \alpha} \psi(t)$ for any $\delta^{\theta \alpha} \leqslant t \leqslant \delta^{\theta^{\prime} \alpha}$, which along with the fact $\psi(t) \leqslant \mu_{x}^{-1} \leqslant c_{M}$ for all $t>0$ yields that $\psi(t) \leqslant c_{M} e^{-2^{-1} c_{9} \delta^{-\theta \alpha}\left(t-\delta^{\theta \alpha}\right)}$ for any $\delta^{\theta \alpha} \leqslant t \leqslant \delta^{\theta^{\prime} \alpha}$. In particular, $\psi\left(\delta^{\theta^{\prime} \alpha}\right) \leqslant$ $c_{M} e^{-2^{-1} c 9 \delta^{-\theta \alpha}\left(\delta^{\theta^{\prime} \alpha}-\delta^{\theta \alpha}\right)}$. On the other hand, according to (2.19), we have $\psi\left(\delta^{\theta^{\prime} \alpha}\right) \geqslant 2 c_{10} \delta^{-d \theta}$. Thus, there is a contradiction between these two inequalities for $\delta$ large enough, and so (2.18) is true.

Next, assume we take $1 \leqslant \delta<r_{G}$ large enough such that (2.18) holds. Since $t \mapsto \psi(t)$ is non-increasing on $(0, \infty)$ and $t_{0} \leqslant \delta^{\theta^{\prime} \alpha}$,

$$
\left(\frac{1}{2 c_{10}} \psi(t)\right)^{-1 / d} \geqslant \delta^{\theta}, \quad \forall \delta^{\theta^{\prime} \alpha} \leqslant t \leqslant \delta^{\alpha} .
$$

Let

$$
\tilde{t}_{0}:=\sup \left\{t>0:\left(\frac{1}{2 c_{10}} \psi(t)\right)^{-1 / d}<\delta / 2\right\} .
$$

By the non-increasing property of $\psi$ on $(0, \infty)$ again, if $\tilde{t}_{0} \leqslant \delta^{\theta^{\prime} \alpha}$, then $\psi(t) \leqslant \psi\left(\tilde{t}_{0}\right)=2 c_{10}(\delta / 2)^{-d} \leqslant$ $c_{11} t^{-d / \alpha}$ for any $\delta^{\theta^{\prime} \alpha} \leqslant t \leqslant \delta^{\alpha}$. This proves (2.8) under the assumption $t_{0} \leqslant \delta^{\theta^{\prime} \alpha}$.

When $\tilde{t}_{0}>\delta^{\theta^{\prime} \alpha}$,

$$
\delta^{\theta} \leqslant\left(\frac{1}{2 c_{10}} \psi(t)\right)^{-1 / d} \leqslant \delta / 2, \quad \forall \delta^{\theta^{\prime} \alpha} \leqslant t \leqslant \tilde{t}_{0} .
$$

Then, taking $r(t)=\left(\frac{1}{2 c_{10}} \psi(t)\right)^{-1 / d}$ in $(2.17)$, we have $\psi^{\prime}(t) \leqslant-c_{12} \psi(t)^{1+\alpha / d}$ for any $\delta^{\theta^{\prime} \alpha} \leqslant t \leqslant \tilde{t}_{0}$. Hence, $\psi(s) \leqslant c_{13}\left(s-\delta^{\theta^{\prime} \alpha}+\psi\left(\delta^{\theta^{\prime} \alpha}\right)^{-\alpha / d}\right)^{-d / \alpha} \leqslant c_{14} s^{-d / \alpha}$ for any $2 \delta^{\theta^{\prime} \alpha} \leqslant s \leqslant \tilde{t}_{0}$. If $\tilde{t}_{0}>\delta^{\alpha}$, then (2.8) holds. If $\delta^{\theta^{\prime} \alpha}<\tilde{t}_{0} \leqslant \delta^{\alpha}$, then, for all $\tilde{t}_{0} \leqslant s \leqslant \delta^{\alpha}, \psi(s) \leqslant \psi\left(\tilde{t}_{0}\right)=2 c_{10}(\delta / 2)^{-d} \leqslant c_{15} s^{-d / \alpha}$, so (2.8) also holds. The proof is complete.

Remark 2.3. By carefully tracking the proof, we can see that the constant $C_{3}>0$ in the statement of Proposition 2.1 can be chosen independently of the choice of $\delta_{0}, \delta>0$. 
2.2. Localization method and moment estimates of the truncated process. In this part, we fix $x_{0} \in V$ and $R \geqslant 1$. Define a symmetric regular Dirichlet form $\left(\hat{D}^{x_{0}, R}, \hat{\mathscr{F}} x_{0}, R\right)$ as follows

$$
\begin{aligned}
\hat{D}^{x_{0}, R}(f, f) & =\sum_{x, y \in V}(f(x)-f(y))^{2} \frac{\hat{w}_{x, y}}{\rho(x, y)^{d+\alpha}} \mu_{x} \mu_{y}, \quad f \in \hat{\mathscr{F}}^{x_{0}, R}, \\
\hat{\mathscr{F}} x_{0}, R & =\left\{f \in L^{2}(V ; \mu): \hat{D}^{x_{0}, R}(f, f)<\infty\right\},
\end{aligned}
$$

where

$$
\hat{w}_{x, y}=\left\{\begin{array}{cl}
w_{x, y}, & \text { if } x \in B\left(x_{0}, R\right) \text { or } y \in B\left(x_{0}, R\right), \\
1, & \text { otherwise. }
\end{array}\right.
$$

In particular, coefficients of the Dirichlet form $\left(\hat{D}^{x_{0}, R}, \hat{\mathscr{F}} x_{0}, R\right)$ outside $B\left(x_{0}, R\right)$ are uniformly bounded. This point is quite important in the following arguments for the exit time estimates from $B\left(x_{0}, R\right)$.

Note that, according to the definition of $\hat{w}_{x, y}$, for any $x \in V$,

$$
\begin{aligned}
& \sum_{y \in V} \frac{\hat{w}_{x, y}}{\rho(x, y)^{d+\alpha}}=\sum_{y \notin B\left(x_{0}, R\right)} \frac{\hat{w}_{x, y}}{\rho(x, y)^{d+\alpha}}+\sum_{y \in B\left(x_{0}, R\right)} \frac{w_{x, y}}{\rho(x, y)^{d+\alpha}} \\
& \leqslant \sup _{z \in B\left(x_{0}, R\right)} \sum_{v \in V} \frac{w_{z, v}}{\rho(z, v)^{d+\alpha}}+\sup _{z \notin B\left(x_{0}, R\right)} \sum_{y \in V: y \neq z} \frac{1}{\rho(z, y)^{d+\alpha}}+\sum_{y \in B\left(x_{0}, R\right)} \frac{w_{x, y}}{\rho(x, y)^{d+\alpha}} \\
& \leqslant \sup _{z \in B\left(x_{0}, R\right)} \sum_{v \in V} \frac{w_{z, v}}{\rho(z, v)^{d+\alpha}}+c_{M} \sup _{z \notin B\left(x_{0}, R\right)} \sum_{k=1}^{\infty} \sum_{y \in V: 2^{k-1} \leqslant \rho(y, z)<2^{k}} \frac{1}{\rho(y, z)^{d+\alpha}} \mu_{y} \\
& \quad+\sum_{y \in B\left(x_{0}, R\right)}\left(\sup _{z \in B\left(x_{0}, R\right)} \sum_{v \in V} \frac{w_{z, v}}{\rho(z, v)^{d+\alpha}}\right) \\
& \leqslant \sup _{z \in B\left(x_{0}, R\right)} \sum_{v \in V} \frac{w_{z, v}}{\rho(z, v)^{d+\alpha}}+c_{M} c_{G} \sum_{k=1}^{\infty} \frac{2^{k d}}{2^{(k-1)(d+\alpha)}}+\sum_{y \in B\left(x_{0}, R\right)} \sup _{z \in B\left(x_{0}, R\right)} \sum_{v \in V} \frac{w_{z, v}}{\rho(z, v)^{d+\alpha}} \\
& \leqslant c_{1}+c_{2}\left(1+R^{d}\right) \sup _{z \in B\left(x_{0}, R\right)}\left(\sum_{v \in V} \frac{w_{z, v}}{\rho(z, v)^{d+\alpha}}\right)=: C\left(x_{0}, R\right)<\infty,
\end{aligned}
$$

where (2.3) was used in the fourth inequality. In particular, by (2.20) and (the second statement in) $\left[23\right.$, Theorem 3.2], the associated Hunt process $\hat{X}^{R}:=\left(\left(\hat{X}_{t}^{R}\right)_{t \geqslant 0},\left(\mathbb{P}_{x}\right)_{x \in V}\right)$ is conservative. Here and in what follows, we omit the index $x_{0}$ for simplicity. The process $\hat{X}^{R}$ is called the localized $\alpha$-stable-like process (with parameters $x_{0} \in V$ and $R \geqslant 1$ ).

We also consider the following truncated Dirichlet form $\left(\hat{D}^{x_{0}, R, R}, \hat{\mathscr{F}}^{x_{0}, R}\right)$ :

$$
\hat{D}^{x_{0}, R, R}(f, f)=\sum_{x, y \in V: \rho(x, y) \leqslant R}(f(x)-f(y))^{2} \frac{\hat{w}_{x, y}}{\rho(x, y)^{d+\alpha}} \mu_{x} \mu_{y}, \quad f \in \hat{\mathscr{F}}^{x_{0}, R} .
$$

Let $\hat{X}^{R, R}:=\left(\left(\hat{X}_{t}^{R, R}\right)_{t \geqslant 0},\left(\mathbb{P}_{x}\right)_{x \in V}\right)$ be the associated Hunt process. In particular, due to (2.20) again, the process $\hat{X}^{R, R}$ is also conservative. Denote by $\hat{p}^{R}(t, x, y)$ and $\hat{p}^{R, R}(t, x, y)$ heat kernels of the processes $\hat{X}^{R}$ and $\hat{X}^{R, R}$, respectively.

The following statement concerns moment estimates of $\hat{X}^{R, R}$. These estimates are key inputs for exit time estimates for the original process $X$ in the next section.

Proposition 2.4. Suppose that there exist $1 \leqslant R_{0}<r_{G}, \theta \in(0,1)$ and $C_{1}, C_{2}>0$ such that for every $R_{0}<R<r_{G}$ and $R^{\theta} \leqslant r \leqslant R$,

$$
\begin{gathered}
\sup _{x \in B\left(x_{0}, 3 R\right)} \sum_{y \in V: \rho(x, y) \leqslant r} \frac{w_{x, y}}{\rho(x, y)^{d+\alpha-2}} \leqslant C_{1} r^{2-\alpha}, \\
\inf _{x \in B\left(x_{0}, 3 R\right)} \mu\left(B^{w}(x, r)\right) \geqslant C_{2} r^{d}
\end{gathered}
$$


and

$$
\sup _{x \in B\left(x_{0}, 3 R\right)} \sum_{y \in B^{w}(x, r)} w_{x, y}^{-1} \leqslant C_{1} r^{d} .
$$

Then for every $\theta^{\prime} \in(\theta, 1)$, there exist constants $R_{1}>R_{0}$ (which depends only on $\theta, \theta^{\prime}$ and $R_{0}$ ) and $C_{3}>0$ (which is independent of $x_{0}, R_{0}$ and $R_{1}$ ) such that for every $R_{1}<R<r_{G}$ and $x \in V$,

$$
\mathbb{E}_{x}\left[\rho\left(\hat{X}_{t}^{R, R}, x\right)\right] \leqslant C_{3} R\left(\frac{t}{R^{\alpha}}\right)^{1 / 2}\left[1+\log \left(\frac{R^{\alpha}}{t}\right)\right], \quad \forall R^{\theta^{\prime} \alpha} \leqslant t \leqslant R^{\alpha},
$$

Proof. Throughout the proof, we first suppose that there exist positive constants $c\left(x_{0}, R\right)$ and $\tilde{c}\left(x_{0}, R\right)$ such that

$$
\tilde{c}\left(x_{0}, R\right) \leqslant \inf _{x, y \in V} \hat{w}_{x, y} \leqslant \sup _{x, y \in V} \hat{w}_{x, y} \leqslant c\left(x_{0}, R\right) .
$$

If (2.25) is not satisfied, then, by taking $w_{x, y}^{\varepsilon}:=w_{x, y}+\varepsilon$ and then letting $\varepsilon \downarrow 0$, we can prove that (2.24) still holds true. Moreover, all the constants in the proof below are independent of $\varepsilon$ unless specifically claimed. The argument below is partly motivated by the method of Bass [12] for diffusions (see also Barlow [6] and Nash [37]), but some non-trivial modifications are required for jump processes.

Step (1): By (2.21), (2.22), (2.23) and the definition of $\hat{w}_{x, y}$, for every $R_{0}<R<r_{G}$ and $R^{\theta} \leqslant r \leqslant R$,

$$
\sup _{x \in V} \sum_{y \in V: \rho(x, y) \leqslant r} \frac{\hat{w}_{x, y}}{\rho(x, y)^{d+\alpha-2}} \leqslant c_{0} r^{2-\alpha},
$$

$\inf _{x \in V} \mu\left(B^{\hat{w}}(x, r)\right) \geqslant c_{1} r^{d}$ and $\sup _{x \in V} \sum_{y \in B^{\hat{w}}(x, r)} \hat{w}_{x, y}^{-1} \leqslant c_{0} r^{d}$, where $B^{\hat{w}}(x, r):=\{z \in V: \rho(z, x) \leqslant$ $\left.r, \hat{w}_{z, x}>0\right\}$. Let $\theta^{\prime} \in(\theta, 1)$ and $\theta_{0}=\left(\theta+\theta^{\prime}\right) / 2$. Taking $\rho=R$ in Proposition 2.1, we find that there exists a constant $\tilde{R}_{0} \geqslant R_{0}$ (which only depends on $\theta$ and $\theta^{\prime}$ ) such that whenever $\tilde{R}_{0}<R<r_{G}$,

$$
\hat{p}^{R, R}(t, x, y) \leqslant c_{2} t^{-d / \alpha}, \quad \forall 2 R^{\theta_{0} \alpha} \leqslant t \leqslant R^{\alpha}, x, y \in V .
$$

For every $t>0$, we define

$$
M(t)=\sum_{y \in V} \rho(x, y) \hat{p}^{R, R}(t, x, y) \mu_{y}, \quad Q(t)=-\sum_{y \in V} \hat{p}^{R, R}(t, x, y)\left[\log \hat{p}^{R, R}(t, x, y)\right] \mu_{y} .
$$

Below, we fix $x \in V$ and set $f_{t}(y)=\hat{p}^{R, R}(t, x, y)$ for all $y \in V$ and $t>0$.

By (2.25), we can obtain upper and lower bounds for $\hat{p}^{R, R}(t, x, y)$ (see [29] for upper bounds on graph or [22] for two-sided estimates in the Euclidean space), which yields that

$$
\begin{aligned}
& \sum_{y, z \in V: \rho(y, z) \leqslant R}\left|f_{t}(y)-f_{t}(z)\right|\left|\log f_{t}(y)-\log f_{t}(z)\right| \frac{\hat{w}_{y, z}}{\rho(y, z)^{d+\alpha}} \mu_{y} \mu_{z} \\
\leqslant & \sum_{y, z \in V: \rho(y, z) \leqslant R}\left(f_{t}(y)+f_{t}(z)\right)\left(\left|\log f_{t}(y)\right|+\left|\log f_{t}(z)\right|\right) \frac{\hat{w}_{y, z}}{\rho(y, z)^{d+\alpha}} \mu_{y} \mu_{z}<\infty .
\end{aligned}
$$

Thus,

$$
\begin{aligned}
& -\sum_{y \in V}\left(\log f_{t}(y)+1\right) \hat{L}^{R, R} f_{t}(y) \mu_{y} \\
& =\frac{1}{2} \sum_{y, z \in V: \rho(y, z) \leqslant R}\left(f_{t}(y)-f_{t}(z)\right)\left(\log f_{t}(y)-\log f_{t}(z)\right) \frac{\hat{w}_{y, z}}{\rho(y, z)^{d+\alpha}} \mu_{y} \mu_{z},
\end{aligned}
$$

where $\hat{L}^{R, R}$ is the generator associated with $\left(\hat{D}^{x_{0}, R, R}, \hat{\mathscr{F}}^{x_{0}, R, R}\right)$, i.e.,

$$
\hat{L}^{R, R} f(x)=\sum_{y \in V: \rho(x, y) \leqslant R}(f(y)-f(x)) \frac{\hat{w}_{x, y}}{\rho(x, y)^{d+\alpha}} \mu_{y} .
$$

Therefore,

$$
Q^{\prime}(t)=-\sum_{y \in V}\left(\log f_{t}(y)+1\right) \hat{L}^{R, R} f_{t}(y) \mu_{y}
$$




$$
=\frac{1}{2} \sum_{y, z \in V: \rho(y, z) \leqslant R}\left(f_{t}(y)-f_{t}(z)\right)\left(\log f_{t}(y)-\log f_{t}(z)\right) \frac{\hat{w}_{y, z}}{\rho(y, z)^{d+\alpha}} \mu_{y} \mu_{z} \geqslant 0 .
$$

In particular, $Q(\cdot)$ is a non-decreasing function on $(0, \infty)$.

On the other hand, for all $\tilde{R}_{0}<R<r_{G}$, by the Cauchy-Schwarz inequality,

$$
\begin{aligned}
M^{\prime}(t)= & \sum_{y \in V} \rho(x, y) \hat{L}^{R, R} f_{t}(y) \mu_{y} \\
= & -\frac{1}{2} \sum_{y, z \in V: \rho(y, z) \leqslant R}(\rho(x, y)-\rho(x, z))\left(f_{t}(y)-f_{t}(z)\right) \frac{\hat{w}_{y, z}}{\rho(y, z)^{d+\alpha}} \mu_{y} \mu_{z} \\
\leqslant & \left(\frac{1}{4} \sum_{y, z \in V: \rho(y, z) \leqslant R}(\rho(x, y)-\rho(x, z))^{2}\left(f_{t}(y)+f_{t}(z)\right) \frac{\hat{w}_{y, z}}{\rho(y, z)^{d+\alpha}} \mu_{y} \mu_{z}\right)^{1 / 2} \\
& \times\left(\sum_{y, z \in V: \rho(y, z) \leqslant R} \frac{\left(f_{t}(y)-f_{t}(z)\right)^{2}}{f_{t}(y)+f_{t}(z)} \frac{\hat{w}_{y, z}}{\rho(y, z)^{d+\alpha}} \mu_{y} \mu_{z}\right)^{1 / 2} \\
\leqslant & \left(\frac{c_{M}}{2} \sup _{z \in V} \sum_{y \in V: \rho(y, z) \leqslant R} \frac{\hat{w}_{y, z}}{\rho(y, z)^{d+\alpha-2}}\right)^{1 / 2} \\
& \times\left(\sum_{y, z \in V: \rho(y, z) \leqslant R} \frac{\left(f_{t}(y)-f_{t}(z)\right)^{2}}{f_{t}(y)+f_{t}(z)} \frac{\hat{w}_{y, z}}{\rho(y, z)^{d+\alpha}} \mu_{y} \mu_{z}\right)^{1 / 2} \\
\leqslant & c_{3} R^{1-\alpha / 2}\left(\sum_{y, z \in V: \rho(y, z) \leqslant R} \frac{\left(f_{t}(y)-f_{t}(z)\right)^{2}}{f_{t}(y)+f_{t}(z)} \frac{\hat{w}_{y, z}}{\rho(y, z)^{d+\alpha}} \mu_{y} \mu_{z}\right)^{1 / 2}
\end{aligned}
$$

where the equality above follows from the fact

$$
\sum_{y, z \in V: \rho(y, z) \leqslant R}\left|f_{t}(y)-f_{t}(z)\right| \frac{\hat{w}_{y, z}}{\rho(y, z)^{d+\alpha-1}}<\infty,
$$

thank to (2.25) again, in the second inequality we used (2.1) and the fact that $\sum_{z \in V} f_{t}(z) \mu_{z} \leqslant 1$ for all $t>0$, and in the last inequality we have used (2.26).

Noting that

$$
\frac{(s-t)^{2}}{s+t} \leqslant(s-t)(\log s-\log t), \quad s, t>0,
$$

we have

$$
\begin{aligned}
& \sum_{y, z \in V: \rho(y, z) \leqslant R} \frac{\left(f_{t}(y)-f_{t}(z)\right)^{2}}{f_{t}(y)+f_{t}(z)} \frac{\hat{w}_{y, z}}{\rho(y, z)^{d+\alpha}} \mu_{y} \mu_{z} \\
& \leqslant \sum_{y, z \in V: \rho(y, z) \leqslant R}\left(f_{t}(y)-f_{t}(z)\right)\left(\log f_{t}(y)-\log f_{t}(z)\right) \frac{\hat{w}_{y, z}}{\rho(y, z)^{d+\alpha}} \mu_{y} \mu_{z}=2 Q^{\prime}(t) .
\end{aligned}
$$

Hence, combining all the estimates above, we arrive at the statement that for all $\tilde{R}_{0}<R<r_{G}$,

$$
M^{\prime}(t) \leqslant \sqrt{2} c_{3} R^{1-\alpha / 2} Q^{\prime}(t)^{1 / 2}, \quad \forall t>0 .
$$

Step (2): (2.27) yields that for all $\tilde{R}_{0}<R<r_{G}$ and $2 R^{\theta_{0} \alpha} \leqslant t \leqslant R^{\alpha}$,

$$
Q(t) \geqslant-\left(\sum_{y \in V} f_{t}(y)\right) \log \left(c_{2} t^{-d / \alpha}\right)=\frac{d}{\alpha} \log t-c_{4},
$$

where $c_{4}>0$ and the conservativeness of $\hat{X}^{R, R}$ was used in the right hand equality. Define

$$
K(t)=d^{-1}\left(Q(t)+c_{4}-\frac{d}{\alpha} \log t\right), \quad t>0 .
$$


Obviously, $K(t) \geqslant 0$ for all $t \in\left[2 R^{\theta_{0} \alpha}, R^{\alpha}\right]$, and

$$
Q^{\prime}(t)=d K^{\prime}(t)+\frac{d}{\alpha t}, \quad t>0 .
$$

Set $T_{0}(R):=0 \vee \sup \left\{t<2 R^{\theta_{0} \alpha}: K(t)<0\right\}$. It is easy to see that $K(t) \geqslant 0$ for all $t \in\left[T_{0}(R), R^{\alpha}\right]$ and $T_{0}(R) \leqslant 2 R^{\theta_{0} \alpha}$. By $(2.28)$ and $(2.29)$, we have for all $t \in\left[T_{0}(R), R^{\alpha}\right]$,

$$
\begin{aligned}
M(t) & =M\left(T_{0}(R)\right)+\int_{T_{0}(R)}^{t} M^{\prime}(s) d s \leqslant M\left(T_{0}(R)\right)+\sqrt{2} c_{3} R^{1-\alpha / 2} \int_{T_{0}(R)}^{t} Q^{\prime}(s)^{1 / 2} d s \\
& =M\left(T_{0}(R)\right)+\sqrt{2} c_{3} R^{1-\alpha / 2} \int_{T_{0}(R)}^{t}\left(d K^{\prime}(s)+\frac{d}{\alpha s}\right)^{1 / 2} d s .
\end{aligned}
$$

Note that, by the mean-value theorem, for every $a \in \mathbb{R}$ and $b>0$ with $a+b \geqslant 0$,

$$
(a+b)^{1 / 2} \leqslant b^{1 / 2}+a /\left(2 b^{1 / 2}\right) .
$$

Then, applying (2.31) in the second term of the right hand side of (2.30) with $a=K^{\prime}(s)$ and $b=\frac{1}{\alpha s}$, we obtain that for all $t \in\left[T_{0}(R), R^{\alpha}\right]$,

$$
\begin{aligned}
M(t) & \leqslant M\left(T_{0}(R)\right)+c_{4} R^{1-\alpha / 2} \int_{T_{0}(R)}^{t} s^{-1 / 2} d s+c_{5} R^{1-\alpha / 2} \int_{T_{0}(R)}^{t} s^{1 / 2} K^{\prime}(s) d s \\
& \leqslant M\left(T_{0}(R)\right)+c_{6} R^{1-\alpha / 2} t^{1 / 2}+c_{5} R^{1-\alpha / 2} \int_{T_{0}(R)}^{t}\left[\left(s^{1 / 2} K(s)\right)^{\prime}-\frac{s^{-1 / 2} K(s)}{2}\right] d s \\
& \leqslant M\left(T_{0}(R)\right)+c_{6} R^{1-\alpha / 2} t^{1 / 2}+c_{5} R^{1-\alpha / 2} t^{1 / 2} K(t),
\end{aligned}
$$

where, in the last inequality, we used the fact that $K(t) \geqslant 0$ for all $t \in\left[T_{0}(R), R^{\alpha}\right]$.

Furthermore, suppose that $T_{0}(R)>0$. Since $Q^{\prime}(t) \geqslant 0$, by (2.28) and the Cauchy-Schwarz inequality, we have

$$
\begin{aligned}
M\left(T_{0}(R)\right) & =\int_{0}^{T_{0}(R)} M^{\prime}(s) d s \leqslant \sqrt{2} c_{3} R^{1-\alpha / 2} \int_{0}^{T_{0}(R)} Q^{\prime}(s)^{1 / 2} d s \\
& \leqslant \sqrt{2} c_{3} R^{1-\alpha / 2} T_{0}(R)^{1 / 2}\left(\int_{0}^{T_{0}(R)} Q^{\prime}(s) d s\right)^{1 / 2} \\
& \leqslant c_{7} R^{1-\alpha\left(1-\theta_{0}\right) / 2}\left(Q\left(T_{0}(R)\right)-(Q(0) \wedge 0)\right)^{1 / 2},
\end{aligned}
$$

where in the last inequality we have used the fact that $T_{0}(R) \leqslant 2 R^{\theta_{0} \alpha}$. By the definition of $T_{0}(R)$, it holds that $K\left(T_{0}(R)\right)=0$, and so $Q\left(T_{0}(R)\right)=(d / \alpha) \log T_{0}(R)-c_{4} \leqslant c_{8}(1+\log R)$, where we have used again $T_{0}(R) \leqslant 2 R^{\theta_{0} \alpha}$. On the other hand, $Q(0)=\lim _{t \rightarrow 0} Q(t)=\log \mu_{x} \geqslant-\log c_{M}$. Thus, we can find $R_{1} \geqslant 1$ large enough such that for all $R>R_{1}$ and $t \in\left[R^{\theta^{\prime} \alpha}, R^{\alpha}\right]$,

$$
\begin{aligned}
M\left(T_{0}(R)\right) & \leqslant c_{9} R^{1-\alpha\left(1-\theta_{0}\right) / 2}(1+\log R)^{1 / 2}=c_{9} R^{1-\alpha / 2} R^{\theta_{0} \alpha / 2}(1+\log R)^{1 / 2} \\
& \leqslant c_{9} R^{1-\alpha / 2} R^{\theta^{\prime} \alpha / 2} \leqslant c_{9} R^{1-\alpha / 2} t^{1 / 2},
\end{aligned}
$$

where in the second inequality we used the fact that $\theta_{0} \in\left(\theta, \theta^{\prime}\right)$, and the last inequality is due to $t \geqslant R^{\theta^{\prime} \alpha}$. Note that $M(0)=0$, so the above estimate still holds when $T_{0}(R)=0$.

Therefore, combining this with (2.32), we arrive at the statement that for all $t \in\left[R^{\theta^{\prime} \alpha}, R^{\alpha}\right]$,

$$
M(t) \leqslant c_{10} R^{1-\alpha / 2} t^{1 / 2}(1+K(t)) .
$$

Step (3): Note that $s(\log s+t) \geqslant-e^{-1-t}$ for all $s>0$ and $t \in \mathbb{R}$. Then, for every $0<a \leqslant 2$, $b \in \mathbb{R}$ and $t>0$,

$$
\begin{aligned}
-Q(t)+a M(t)+b & =\sum_{y \in V} f_{t}(y)\left(\log f_{t}(y)+a \rho(x, y)+b\right) \mu_{y} \\
& \geqslant-\sum_{y \in V} \exp (-1-a \rho(x, y)-b) \mu_{y} \geqslant-c_{11} e^{-b} a^{-d},
\end{aligned}
$$


where the equality above follows from the conservativeness of $X^{R, R}$, and in the last inequality we used the fact that

$$
\sum_{y \in V} e^{-a \rho(x, y)} \mu_{y} \leqslant c_{M}+\sum_{k=1}^{\infty} \sum_{y \in B\left(x, 2^{k}\right) \backslash B\left(x, 2^{k-1}\right)} e^{-a 2^{k-1}} \mu_{y} \leqslant c_{M}+c_{G} \sum_{k=1}^{\infty} 2^{d k} e^{-a 2^{k-1}} \leqslant C a^{-d}
$$

for all $0<a \leqslant 2$ (see $[6$, line $6-7$ in p. 3056]).

According to (2.27), we could find $R_{1}>\tilde{R}_{0}$ large enough such that for all $R_{1}<R<r_{G}$ and $t \in\left[R^{\theta^{\prime} \alpha}, R^{\alpha}\right]$,

$$
\begin{aligned}
M(t) & =\sum_{y \in V} \rho(x, y) f_{t}(y) \mu_{y} \geqslant \sum_{y \in V: \rho(x, y)>0} f_{t}(y) \mu_{y}=1-\mathbb{P}_{x}\left(\hat{X}_{t}^{R, R}=x\right) \\
& \geqslant 1-c_{2} t^{-d / \alpha} \geqslant 1-c_{2} R^{-\theta^{\prime} d}>1 / 2 .
\end{aligned}
$$

Then, choosing $a=1 / M(t)$ and $e^{b}=M(t)^{d}=a^{-d}$ in (2.34), we have $-Q(t)+1+d \log M(t) \geqslant-c_{11}$, which implies that for all $R_{1}<R<r_{G}$ and $t \in\left[R^{\theta^{\prime} \alpha}, R^{\alpha}\right], M(t) \geqslant c_{12} \exp (Q(t) / d)$. This along with the definition of $K(t)$ yields that

$$
M(t) \geqslant c_{12} \exp (Q(t) / d) \geqslant c_{13} t^{1 / \alpha} e^{K(t)} .
$$

Combining (2.33) with (2.35), we obtain that for all $t \in\left[R^{\theta^{\prime} \alpha}, R^{\alpha}\right]$,

$$
e^{K(t)} \leqslant c_{14} R^{1-\alpha / 2}(1+K(t)) t^{1 / 2-1 / \alpha},
$$

which is equivalent to

$$
K(t) \leqslant c_{15}\left[1+\log \left(\frac{R^{\alpha}}{t}\right)+\log (1+K(t))\right] .
$$

This implies that for all $R_{1}<R<r_{G}$ and $t \in\left[R^{\theta^{\prime} \alpha}, R^{\alpha}\right]$,

$$
K(t) \leqslant c_{16}\left[1+\log \left(\frac{R^{\alpha}}{t}\right)\right] .
$$

The inequality above along with (2.33) further gives us that for all $R_{1}<R<r_{G}$ and $t \in\left[R^{\theta^{\prime} \alpha}, R^{\alpha}\right]$,

$$
M(t) \leqslant c_{17} R^{1-\alpha / 2} t^{1 / 2}\left[1+\log \left(\frac{R^{\alpha}}{t}\right)\right] \leqslant c_{18} R\left(\frac{t}{R^{\alpha}}\right)^{1 / 2}\left[1+\log \left(\frac{R^{\alpha}}{t}\right)\right] .
$$

The proof is complete.

\section{3. $\alpha$-STABLE-LIKE PROCESSES ON GRAPHS}

Let $(D, \mathscr{F})$ be a regular symmetric Dirichlet form on $L^{2}(V ; \mu)$ given in the beginning of Section 2, i.e.,

$$
D(f, f)=\frac{1}{2} \sum_{x, y \in V}(f(x)-f(y))^{2} \frac{w_{x, y}}{\rho(x, y)^{d+\alpha}} \mu_{x} \mu_{y}, \quad f \in \mathscr{F}=\left\{f \in L^{2}(V ; \mu): D(f, f)<\infty\right\},
$$

where $\alpha \in(0,2)$ and $\left\{w_{x, y}: x, y \in V\right\}$ is a sequence such that $w_{x, x}=0$ for all $x \in V, w_{x, y} \geqslant 0$ and $w_{x, y}=w_{y, x}$ for all $x \neq y$, and $(2.3)$ holds. Let $X:=\left(\left(X_{t}\right)_{t \geqslant 0},\left(\mathbb{P}_{x}\right)_{x \in V}\right)$ be the associated symmetric $\alpha$-stable-like process associated with $(D, \mathscr{F})$.

In this section, we will derive exit time estimates for the process $X$ and the Hölder regularity of the associated caloric functions. Both statements are crucial to establish the weak convergence of $\alpha$-stable-like processes in the next section.

3.1. Estimates of exit time: for any fixed starting point. In this part, we are concerned on exit time estimates of the process $X$ for any fixed starting point. The main statement is as follows.

Proposition 3.1. Assume that there exist $R_{0} \geqslant 1, \theta \in(0,1)$ and $C_{1}>0$ such that for every $R_{0}<R<r_{G}$ and $R^{\theta} \leqslant r \leqslant R,(2.21),(2.22)$ and (2.23) as well as

$$
\sup _{x \in B\left(x_{0}, R\right)} \sum_{y \in V: \rho(x, y)>R} \frac{w_{x, y}}{\rho(x, y)^{d+\alpha}} \leqslant C_{1} R^{-\alpha}
$$

hold. Then 
(i) for any $\theta^{\prime} \in(\theta, 1)$, there exist constants $R_{1} \geqslant 1$ (which depends only on $\theta, \theta^{\prime}$ and $R_{0}$ ) and $C_{2}>0$ (which is independent of $x_{0}, R_{0}$ and $R_{1}$ ) such that for every $R_{1}<R<r_{G}$,

$$
\mathbb{P}_{x_{0}}\left(\tau_{B\left(x_{0}, R\right)} \leqslant t\right) \leqslant C_{2}\left(\frac{t}{R^{\alpha}}\right)^{1 / 2}\left[1 \vee \log \left(\frac{R^{\alpha}}{t}\right)\right], \quad \forall t \geqslant R^{\theta^{\prime} \alpha} .
$$

(ii) for any $\varepsilon>0$, there exist constants $R_{2} \geqslant 1$ (which depends only on $\theta, R_{0}$ and $\varepsilon$ ) and $C_{3}(\varepsilon)>0$ (which is independent of $x_{0}, R_{0}$ and $R_{2}$ ) such that for all $R_{2}<R<r_{G}$,

$$
\mathbb{P}_{x_{0}}\left(\tau_{B\left(x_{0}, R\right)} \leqslant t\right) \leqslant \varepsilon+\frac{C_{3}(\varepsilon) t}{R^{\alpha}}, \quad \forall t>0
$$

In particular, the process $X$ is conservative.

To prove Proposition 3.1, we will make use of Proposition 2.4. We adopt notations from Subsection 2.2. Fix $x_{0} \in V$ and $R \geqslant 1$. According to the definition of $\left(\hat{D}^{x_{0}, R}, \hat{\mathscr{F}} x_{0}, R\right)$, we have

$$
\mathbb{P}_{x_{0}}\left(\tau_{B\left(x_{0}, R\right)} \leqslant t\right)=\mathbb{P}_{x_{0}}\left(\hat{\tau}_{B\left(x_{0}, R\right)}^{R} \leqslant t\right),
$$

where $\tau_{A}:=\inf \left\{t>0: X_{t} \notin A\right\}$ and $\hat{\tau}_{A}^{R}:=\inf \left\{t \geqslant 0: \hat{X}_{t}^{R} \notin A\right\}$ for any subset $A \subseteq V$. This is, for fixed $x_{0} \in V$, the distribution of exit time for the process $X$ exiting from $B\left(x_{0}, R\right)$ is the same as that for the corresponding localized process $\left(X_{t}^{R}\right)_{t \geqslant 0}$ (with parameters $x_{0}$ and $R$ ).

In order to apply Proposition 2.4 and obtain $\mathbb{P}_{x_{0}}\left(\hat{\tau}_{B\left(x_{0}, R\right)}^{R} \leqslant t\right)$, we now use the truncation idea. In the following, we denote by $\left(\hat{P}_{t}^{R, B\left(x_{0}, R\right)}\right)_{t \geqslant 0}$ and $\left(\hat{P}_{t}^{R, R, B\left(x_{0}, R\right)}\right)_{t \geqslant 0}$ Dirichlet semigroups of the processes $\hat{X}^{R}$ and $\hat{X}^{R, R}$ exiting $B\left(x_{0}, R\right)$, respectively. Let $\hat{\tau}_{A}^{R, R}=\inf \left\{t \geqslant 0: \hat{X}_{t}^{R, R} \notin A\right\}$ for any $A \subseteq V$.

Lemma 3.2. There exists a constant $C_{1}>0$ such that for every $f \in L^{2}(V ; \mu), t>0$ and $x \in$ $B\left(x_{0}, R\right)$,

$$
\left|\hat{P}_{t}^{R, R, B\left(x_{0}, R\right)} f(x)-\hat{P}_{t}^{R, B\left(x_{0}, R\right)} f(x)\right| \leqslant C_{1} t\left(\sup _{y \in B\left(x_{0}, R\right)} J(y, R)\right)\left(\sup _{z \in B\left(x_{0}, R\right)}|f(z)|\right),
$$

where

$$
J(y, R)=\sum_{z \in V: \rho(y, z)>R} \frac{w_{y, z}}{\rho(y, z)^{d+\alpha}} \mu_{z}, \quad y \in B\left(x_{0}, R\right) .
$$

In particular, it holds that for any $t>0$ and $x \in B\left(x_{0}, R\right)$,

$$
\left|\mathbb{P}_{x}\left(\hat{\tau}_{B\left(x_{0}, R\right)}^{R, R} \leqslant t\right)-\mathbb{P}_{x}\left(\hat{\tau}_{B\left(x_{0}, R\right)}^{R} \leqslant t\right)\right| \leqslant C_{1} t \sup _{y \in B\left(x_{0}, R\right)} J(y, R) .
$$

Proof. Let $T_{R}^{R}=\inf \left\{t>0: \rho\left(\hat{X}_{t-}^{R}, \hat{X}_{t}^{R}\right)>R\right\}$. By $(2.20), \sup _{y \in V} \sum_{z \in V: \rho(z, y)>R} \frac{\hat{w}_{z, y}}{\rho(z, y)^{d+\alpha}} \mu_{z}<$ $\infty$. Then, by Meyer's construction of $\hat{X}^{R}$ (see [10, Section 3.1]), $\hat{X}_{t}^{R}$ and $\hat{X}_{t}^{R, R}$ enjoy the same distribution if $t<T_{R}^{R}$. Hence, for any $f \in L^{2}(V ; \mu)$,

$$
\begin{aligned}
& \left|\hat{P}_{t}^{R, R, B\left(x_{0}, R\right)} f(x)-\hat{P}_{t}^{R, B\left(x_{0}, R\right)} f(x)\right| \\
& =\left|\mathbb{E}_{x}\left(f\left(\hat{X}_{t}^{R}\right): t \leqslant \hat{\tau}_{B\left(x_{0}, R\right)}^{R}\right)-\mathbb{E}_{x}\left(f\left(\hat{X}_{t}^{R, R}\right): t \leqslant \hat{\tau}_{B\left(x_{0}, R\right)}^{R, R}\right)\right| \\
& \leqslant \sup _{z \in B\left(x_{0}, R\right)}|f(z)|\left[\mathbb{P}_{x}\left(T_{R}^{R} \leqslant t \leqslant \hat{\tau}_{B\left(x_{0}, R\right)}^{R}\right)+\mathbb{P}_{x}\left(T_{R}^{R} \leqslant t \leqslant \hat{\tau}_{B\left(x_{0}, R\right)}^{R, R}\right)\right] \\
& \leqslant 2\left(\sup _{z \in B\left(x_{0}, R\right)}|f(z)|\right) \mathbb{P}_{x}\left(T_{R}^{R} \leqslant t, \hat{X}_{s}^{R, R} \in B\left(x_{0}, R\right) \text { for all } s \in\left[0, T_{R}^{R}\right]\right) .
\end{aligned}
$$

According to [10, Lemma 3.1(a)],

$$
\mathbb{P}_{x}\left(T_{R}^{R} \in d t \mid \mathscr{F}^{\hat{X}^{R, R}}\right)=\hat{J}\left(\hat{X}_{t}^{R, R}, R\right) \exp \left(-\int_{0}^{t} \hat{J}\left(\hat{X}_{s}^{R, R}, R\right) d s\right) d t,
$$

where $\mathscr{F}^{\hat{X}^{R, R}}$ denotes the $\sigma$-algebra generated by $\hat{X}^{R, R}$, and

$$
\hat{J}(y, R)=\sum_{z \in V: \rho(y, z)>R} \frac{\hat{w}_{y, z}}{\rho(y, z)^{d+\alpha}} \mu_{z}, \quad y \in B\left(x_{0}, R\right) .
$$


In particular, by the definition of $\hat{w}_{x, y}, J(y, R)=\hat{J}(y, R)$ for all $y \in B\left(x_{0}, R\right)$. Therefore,

$$
\begin{aligned}
& \mathbb{P}_{x}\left(T_{R}^{R} \leqslant t, \hat{X}_{s}^{R, R} \in B\left(x_{0}, R\right) \text { for all } s \in\left[0, T_{R}^{R}\right]\right) \\
& \leqslant \mathbb{E}_{x}\left[\int_{0}^{t} J\left(\hat{X}_{r}^{R, R}, R\right) \exp \left(-\int_{0}^{r} J\left(\hat{X}_{s}^{R, R}, R\right) d s\right) \mathbb{1}_{\left\{\hat{X}_{s}^{R, R} \in B\left(x_{0}, R\right) \text { for all } s \in[0, r]\right\}} d r\right] \\
& \leqslant c_{1} t \sup _{y \in B\left(x_{0}, R\right)} J(y, R) .
\end{aligned}
$$

Combining all the estimates above, we can obtain (3.5). (3.7) is a direct consequence of (3.5) by taking $f \equiv 1$ on $B\left(x_{0}, R\right)$.

Proof of Proposition 3.1. Step (1): It immediately follows from (3.1) that

$$
\sup _{y \in B\left(x_{0}, R\right)} J(y, R) \leqslant c_{1} R^{-\alpha},
$$

where $J(y, R)$ is defined by (3.6).

Since $(2.21),(2.22)$ and $(2.23)$ are true, by $(2.24)$, for any $\theta^{\prime} \in(\theta, 1)$, there is a constant $\tilde{R}_{1} \geqslant 1$ such that for all $\tilde{R}_{1}<R<r_{G}$ and $x \in V$,

$$
\mathbb{E}_{x}\left[\rho\left(\hat{X}_{t}^{R, R}, x\right)\right] \leqslant c_{2} R\left(\frac{t}{R^{\alpha}}\right)^{1 / 2}\left[1+\log \left(\frac{R^{\alpha}}{t}\right)\right], \quad \forall R^{\theta^{\prime} \alpha} \leqslant t \leqslant R^{\alpha} .
$$

Hence, by the Markov inequality, for all $x \in V, \tilde{R}_{1}<R<r_{G}$ and $R^{\theta^{\prime} \alpha} \leqslant t \leqslant R^{\alpha} / 2$,

$$
\sup _{s \in[t, 2 t]} \mathbb{P}_{x}\left(\rho\left(\hat{X}_{s}^{R, R}, x\right)>\frac{R}{2}\right) \leqslant c_{3}\left(\frac{t}{R^{\alpha}}\right)^{1 / 2}\left[1+\log \left(\frac{R^{\alpha}}{t}\right)\right] .
$$

Therefore, for all $\tilde{R}_{1}<R<r_{G}$ and $R^{\theta^{\prime} \alpha} \leqslant t \leqslant R^{\alpha} / 2$,

$$
\begin{aligned}
& \mathbb{P}_{x_{0}}\left(\hat{\tau}_{B\left(x_{0}, R\right)}^{R, R} \leqslant t\right) \leqslant \mathbb{P}_{x_{0}}\left(\hat{\tau}_{B\left(x_{0}, R\right)}^{R, R} \leqslant t ; \rho\left(\hat{X}_{2 t}^{R, R}, x_{0}\right) \leqslant \frac{R}{2}\right)+\mathbb{P}_{x_{0}}\left(\rho\left(\hat{X}_{2 t}^{R, R}, x_{0}\right)>\frac{R}{2}\right) \\
& \leqslant \mathbb{E}_{x_{0}}\left[\mathbb{1}_{\left\{\hat{\tau}_{B\left(x_{0}, R\right)}^{R, R} \leqslant t\right\}} \mathbb{P}_{\hat{X}_{\hat{\tau}_{B\left(x_{0}, R\right)}^{R, R}}^{R, R}}\left(\rho\left(\hat{X}_{2 t-\hat{\tau}_{B\left(x_{0}, R\right)}^{R, R}}^{R, R}, \hat{X}_{0}^{R, R}\right)>\frac{R}{2}\right)\right] \\
& +c_{3}\left(\frac{t}{R^{\alpha}}\right)^{1 / 2}\left[1+\log \left(\frac{R^{\alpha}}{t}\right)\right] \\
& \leqslant \sup _{y \in V} \sup _{s \in[t, 2 t]} \mathbb{P}_{y}\left(\rho\left(\hat{X}_{s}^{R, R}, y\right)>\frac{R}{2}\right)+c_{3}\left(\frac{t}{R^{\alpha}}\right)^{1 / 2}\left[1+\log \left(\frac{R^{\alpha}}{t}\right)\right] \\
& \leqslant 2 c_{3}\left(\frac{t}{R^{\alpha}}\right)^{1 / 2}\left[1+\log \left(\frac{R^{\alpha}}{t}\right)\right] \text {. }
\end{aligned}
$$

Combining this with (3.4), (3.7) and (3.8) yields that for all $\tilde{R}_{1}<R<r_{G}$ and $R^{\theta^{\prime} \alpha} \leqslant t \leqslant R^{\alpha} / 2$,

$$
\mathbb{P}_{x_{0}}\left(\tau_{B\left(x_{0}, R\right)} \leqslant t\right) \leqslant 2 c_{3}\left(\frac{t}{R^{\alpha}}\right)^{1 / 2}\left[1+\log \left(\frac{R^{\alpha}}{t}\right)\right]+\frac{c_{4} t}{R^{\alpha}} \leqslant c_{5}\left(\frac{t}{R^{\alpha}}\right)^{1 / 2}\left[1 \vee \log \left(\frac{R^{\alpha}}{t}\right)\right] .
$$

Thus, (3.2) has been verified for all $R^{\theta^{\prime} \alpha} \leqslant t \leqslant R^{\alpha} / 2$. When $t>R^{\alpha} / 2$, it holds that

$$
\mathbb{P}_{x_{0}}\left(\tau_{B\left(x_{0}, R\right)} \leqslant t\right) \leqslant 1 \leqslant\left(\frac{2 t}{R^{\alpha}}\right)^{1 / 2}\left[1 \vee \log \left(\frac{R^{\alpha}}{t}\right)\right]
$$

Hence we prove (3.2).

Step (2): Fix $\theta^{\prime} \in(\theta, 1)$. By (3.2) and Young's inequality, there is a constant $\tilde{R}_{1} \geqslant 1$ such that for every $\tilde{R}_{1}<R<r_{G}, t \geqslant R^{\theta^{\prime} \alpha}$ and $\varepsilon>0, \mathbb{P}_{x_{0}}\left(\tau_{B\left(x_{0}, R\right)} \leqslant t\right) \leqslant 2^{-1} \varepsilon+c_{6}(\varepsilon) t R^{-\alpha}$. If $0<t \leqslant R^{\theta^{\prime} \alpha}$, then, taking $\tilde{R}_{2}(\varepsilon) \geqslant \tilde{R}_{1}$ large enough, we obtain that for all $\tilde{R}_{2}(\varepsilon) \leqslant R<r_{G}$, $\mathbb{P}_{x_{0}}\left(\tau_{B\left(x_{0}, R\right)} \leqslant t\right) \leqslant \mathbb{P}_{x_{0}}\left(\tau_{B\left(x_{0}, R\right)} \leqslant R^{\theta^{\prime} \alpha}\right) \leqslant 2^{-1} \varepsilon+c_{6}(\varepsilon) R^{-\left(1-\theta^{\prime}\right) \alpha} \leqslant \varepsilon$. Combining both estimates above together, we know that for all $\tilde{R}_{2}(\varepsilon)<R<r_{G}$ and $t>0, \mathbb{P}_{x_{0}}\left(\tau_{B\left(x_{0}, R\right)} \leqslant t\right) \leqslant \varepsilon+c_{7}(\varepsilon) t R^{-\alpha}$, which implies that (3.3) holds. 
3.2. Estimates of exit time: locally uniform with respect to the starting point. For our later use, we need exit time estimates for the process, which are locally uniform with respect to the starting point. We first present the following assumption on $\left\{w_{x, y}: x, y \in V\right\}$, which is regarded as the locally uniform version of assumptions in Proposition 3.1. For any $x, z \in V$ and $r>0$, denote $B_{z}^{w}(x, r):=\left\{u \in B(x, r): w_{u, z}>0\right\}$. In particular, $B_{x}^{w}(x, r)=B^{w}(x, r)$.

Assumption (Exi. $(\theta)$ ). Suppose that for some fixed $\theta \in(0,1)$ and $0 \in V$, there exist constants $R_{0} \geqslant 1, c_{0} \in(1 / 2,1)$ and $C_{1}, C_{2}>0$ such that the following hold.

(i) For every $R_{0}<R<r_{G}$ and $R^{\theta} / 2 \leqslant r \leqslant 2 R$,

$$
\begin{gathered}
\sup _{x \in B(0,6 R)} \sum_{y \in V: \rho(x, y) \leqslant r} \frac{w_{x, y}}{\rho(x, y)^{d+\alpha-2}} \leqslant C_{1} r^{2-\alpha}, \\
\mu\left(B_{z}^{w}(x, r)\right) \geqslant c_{0} \mu(B(x, r)), \quad \forall x, z \in B(0,6 R)
\end{gathered}
$$

and

$$
\sup _{x \in B(0,6 R)} \sum_{y \in B^{w}\left(x, c_{*} r\right)} w_{x, y}^{-1} \leqslant C_{1} r^{d}
$$

where $c_{*}:=8 c_{G}^{2 / d}$.

(ii) For every $R_{0}<R<r_{G}$ and $r \geqslant R^{\theta} / 2$,

$$
\sup _{x \in B(0,6 R)} \sum_{y \in V: \rho(x, y)>r} \frac{w_{x, y}}{\rho(x, y)^{d+\alpha}} \leqslant C_{1} r^{-\alpha} .
$$

Then, we have the following statement.

Theorem 3.3. Suppose that Assumption (Exi. $(\theta)$ ) holds with some constant $\theta \in(0,1)$. Then, for every $\theta^{\prime} \in(\theta, 1)$, there exist constants $R_{1} \geqslant 1, \delta \in(\theta, 1)$ and $C_{0}, C_{1}, C_{2}>0$ such that for all $R_{1}<R<r_{G} /\left(2 c_{*}\right)$ and $R^{\delta} \leqslant r \leqslant R$,

$$
\sup _{x \in B(0,2 R)} \mathbb{P}_{x}\left(\tau_{B(x, r)} \leqslant C_{0} r^{\alpha}\right) \leqslant \frac{1}{4}
$$

$$
\sup _{x \in B(0,2 R)} \mathbb{P}_{x}\left(\tau_{B(x, r)} \leqslant t\right) \leqslant C_{1}\left(\frac{t}{r^{\alpha}}\right)^{1 / 2}\left[1 \vee \log \left(\frac{r^{\alpha}}{t}\right)\right], \quad \forall t \geqslant r^{\theta^{\prime} \alpha},
$$

and

$$
C_{2} r^{\alpha} \leqslant \inf _{x \in B(0,2 R)} \mathbb{E}_{x}\left[\tau_{B(x, r)}\right] \leqslant \sup _{x \in B(0,2 R)} \mathbb{E}_{x}\left[\tau_{B(x, r)}\right] \leqslant C_{1} r^{\alpha} .
$$

To prove Theorem 3.3, we begin with the following simple lemma.

Lemma 3.4. Suppose for some constant $\theta \in(0,1)$, (3.10) and (3.11) in Assumption (Exi.( $\theta))(\mathrm{i})$ hold. Then there exists a constant $C_{1}>0$, independent of $R_{0}$, such that for every $R_{0}<R<r_{G} /\left(2 c_{*}\right)$ and $R^{\theta} / 2 \leqslant r \leqslant 2 R$,

$$
\inf _{x \in B(0,6 R)} \sum_{y \in V: \rho(x, y)>3 r} \frac{w_{x, y}}{\rho(x, y)^{d+\alpha}} \geqslant C_{1} r^{-\alpha} .
$$

Here $c_{*}$ is the constant in Assumption (Exi. $\left.(\theta)\right)(\mathrm{i})$.

Proof. Noting that $c_{*}>4$, for every $x \in V$ and $1 \leqslant r<r_{G} / c_{*}$, we have

$$
\sum_{y \in V: 3 r<\rho(x, y) \leqslant c_{*} r, w_{x, y}>0} \mu_{y} \geqslant \mu\left(B^{w}\left(x, c_{*} r\right)\right)-\mu(B(x, 4 r)) \geqslant c_{0} c_{G}^{-1}\left(c_{*} r\right)^{d}-c_{G}(4 r)^{d} \geqslant c_{1} r^{d},
$$

where we have used (2.2) and (3.10).

On the other hand, for every $R_{0}<R<r_{G} /\left(2 c_{*}\right), x \in B(0,6 R)$ and $R^{\theta} / 2 \leqslant r \leqslant 2 R$,

$$
\sum_{y \in V: 3 r<\rho(x, y) \leqslant c_{*} r, w_{x, y}>0} \mu_{y} \leqslant\left(\sum_{y \in B^{w}\left(x, c^{*} r\right)} w_{x, y}^{-1} \mu_{y}\right)^{1 / 2}\left(\sum_{y \in V: 3 r<\rho(x, y) \leqslant c_{*} r} w_{x, y} \mu_{y}\right)^{1 / 2}
$$




$$
\leqslant c_{2} r^{d / 2}\left(\sum_{y \in V: 3 r<\rho(x, y) \leqslant c_{*} r} w_{x, y}\right)^{1 / 2}
$$

where in the first inequality we have applied the Cauchy-Schwarz inequality, and we used (3.11) in the last inequality.

Combining the previous two estimates together yields the statement that for every $R_{0}<R<$ $r_{G} /\left(2 c_{*}\right), x \in B(0,6 R)$ and $R^{\theta} / 2 \leqslant r \leqslant 2 R, \sum_{y \in V: 3 r<\rho(x, y) \leqslant c_{*} r} w_{x, y} \geqslant c_{3} r^{d}$, and so

$$
\sum_{y \in V: \rho(x, y)>3 r} \frac{w_{x, y}}{\rho(x, y)^{d+\alpha}} \geqslant \sum_{y \in V: 3 r<\rho(x, y) \leqslant c_{*} r} \frac{w_{x, y}}{\rho(x, y)^{d+\alpha}} \geqslant\left(c_{*} r\right)^{-d-\alpha} \sum_{y \in V: 3 r<\rho(x, y) \leqslant c_{*} r} w_{x, y} \geqslant c_{4} r^{-\alpha} .
$$

Thus, (3.16) is proved.

Proof of Theorem 3.3. Suppose that Assumption (Exi. $(\theta)$ ) holds with some $\theta \in(0,1)$ and $R_{0} \geqslant 1$. Then, for any $\theta<\theta_{1}<\theta^{\prime}<1, R_{0}<R<r_{G}$ and $R^{\delta} \leqslant s \leqslant R$ with $\delta=\theta / \theta_{1}$, we know that (2.21), (2.23) and (3.1) hold uniformly (that is, they hold with uniform constants) for every $s^{\theta_{1}} \leqslant r \leqslant s$ and $x_{0} \in B(0,2 R)$. Hence, according to (3.2) and (3.3), we obtain that for every $\theta^{\prime} \in(\theta, 1)$, there exists a constant $R_{1} \geqslant R_{0}$ such that for each $R_{1}<R<r_{G}$ and $R^{\delta} \leqslant r \leqslant R,(3.14)$ and

$$
\sup _{x \in B(0,2 R)} \mathbb{P}_{x}\left(\tau_{B(x, r)} \leqslant t\right) \leqslant \frac{1}{8}+\frac{c_{1} t}{r^{\alpha}}, \quad \forall t>0
$$

hold true. In particular, taking $t=\left(8 c_{1}\right)^{-1} r^{\alpha}$ in (3.17), we get (3.13) immediately.

Let $C_{0}$ be the constant in (3.13). For any $R>R_{1}, x \in B(0,2 R)$ and $R^{\delta} \leqslant r \leqslant R$, we have

$$
\begin{aligned}
\mathbb{E}_{x}\left[\tau_{B(x, r)}\right] & =\int_{0}^{\infty} \mathbb{P}_{x}\left(\tau_{B(x, r)}>s\right) d s \geqslant \int_{0}^{C_{0} r^{\alpha}} \mathbb{P}_{x}\left(\tau_{B(x, r)}>s\right) d s \\
& \geqslant C_{0} r^{\alpha} \mathbb{P}_{x}\left(\tau_{B(x, r)}>C_{0} r^{\alpha}\right) \geqslant \frac{3 C_{0} r^{\alpha}}{4} .
\end{aligned}
$$

This gives us the first inequality in (3.15). On the other hand, let $c_{*}$ be the constant in Assumption (Exi. $(\theta))(\mathrm{i})$. By the Lévy system (see [25, Appendix A]), for any $R_{1}<R<r_{G} /\left(2 c_{*}\right), x \in B(0,2 R)$ and $R^{\delta} \leqslant r \leqslant R$,

$$
\begin{aligned}
1 & \geqslant \mathbb{P}_{x}\left(X_{\tau_{B(x, r)}} \notin B(x, 2 r)\right)=\mathbb{E}_{x}\left[\int_{0}^{\tau_{B(x, r)}} \sum_{y \in V: \rho(x, y)>2 r} \frac{w_{X_{s}, y}}{\rho\left(X_{s}, y\right)^{d+\alpha}} \mu_{y} d s\right] \\
& \geqslant c_{M}^{-1} \mathbb{E}_{x}\left[\int_{0}^{\tau_{B(x, r)}} \sum_{y \in V: \rho\left(y, X_{s}\right)>3 r} \frac{w_{X_{s}, y}}{\rho\left(X_{s}, y\right)^{d+\alpha}} d s\right] \\
& \geqslant c_{M}^{-1}\left(\inf _{v \in B(0,2 R+r)} \sum_{y \in V: \rho(y, v)>3 r} \frac{w_{v, y}}{\rho(v, y)^{d+\alpha}}\right) \mathbb{E}_{x}\left[\tau_{B(x, r)}\right] \geqslant c_{2} r^{-\alpha} \mathbb{E}_{x}\left[\tau_{B(x, r)}\right],
\end{aligned}
$$

where in the last inequality we have used (3.16), also thanks to the fact that $\delta=\theta / \theta_{1}>\theta$. Thus, we also prove the third inequality in (3.15).

Finally, we remark that, when $\alpha \in(0,1)$ one can obtain the probability estimate (3.3) for the exit time in a more direct way under the following assumption.

Assumption (Exi. $\left.(\theta)^{\prime}\right)$. Suppose that for some fixed $\theta \in(0,1)$ and $0 \in V$, there exist constants $R_{0} \geqslant 1$ and $C_{1}>0$ such that

(i) for every $R_{0}<R<r_{G}$ and $R^{\theta} / 2 \leqslant r \leqslant 2 R$,

$$
\sup _{x \in B(0,6 R)} \sum_{y \in V: \rho(x, y) \leqslant r} \frac{w_{x, y}}{\rho(x, y)^{d+\alpha-1}} \leqslant C_{1} r^{1-\alpha}
$$

and (3.11) hold.

(ii) (ii) in Assumption (Exi. $(\theta))$ is satisfied. 
Proposition 3.5. Under (3.18) and (ii) in Assumption (Exi. $(\theta)$ ), there exist constants $R_{1}>R_{0}$ and $C_{1}>0$, which are independent of $R_{0}$, such that for all $R_{1}<R<r_{G}, x \in B(0,2 R), R^{\theta} \leqslant r \leqslant R$ and $t>0$,

$$
\mathbb{P}_{x}\left(\tau_{B(x, r)} \leqslant t\right) \leqslant \frac{C_{1} t}{r^{\alpha}} .
$$

Proof. Fix $x \in B(0,2 R)$. Given $f \in C_{b}^{1}([0, \infty))$ with $f(0)=0$ and $f(u)=1$ for all $u \geqslant 1$, we set $f_{x, r}(z)=f\left(\frac{\rho(z, x)}{r}\right)$ for any $z \in V$ and $r>0$. For any $r>0$,

$$
\left\{f_{x, r}\left(X_{t}\right)-f_{x, r}\left(X_{0}\right)-\int_{0}^{t} L f_{x, r}\left(X_{s}\right) d s, t \geqslant 0\right\}
$$

is a local martingale. Then, for any $t>0$ and $x \in V$,

$$
\mathbb{P}_{x}\left(\tau_{B(x, r)} \leqslant t\right) \leqslant \mathbb{E}_{x} f_{x, r}\left(X_{t \wedge \tau_{B(x, r)}}\right)=\mathbb{E}_{x}\left[\int_{0}^{t \wedge \tau_{B(x, r)}} L f_{x, r}\left(X_{s}\right) d s\right] \leqslant t \sup _{z \in B(x, r)} L f_{x, r}(z),
$$

where we used the fact that $f_{x, r}(x)=0$ in the equality above.

Furthermore, for any $x \in V$ and $z \in B(x, r)$,

$$
\begin{aligned}
L f_{x, r}(z)= & \sum_{y \in V}\left(f_{x, r}(y)-f_{x, r}(z)\right) \frac{w_{y, z}}{\rho(z, y)^{d+\alpha}} \mu_{y} \\
= & \sum_{y \in V: \rho(y, z) \leqslant r}\left(f_{x, r}(y)-f_{x, r}(z)\right) \frac{w_{y, z}}{\rho(y, z)^{d+\alpha}} \mu_{y} \\
& +\sum_{y \in V: \rho(y, z)>r}\left(f_{x, r}(y)-f_{x, r}(z)\right) \frac{w_{y, z}}{\rho(y, z)^{d+\alpha}} \mu_{y} \\
\leqslant & c_{1}\left(r^{-1} \sum_{y \in V: \rho(z, y) \leqslant r} \frac{w_{y, z}}{\rho(y, z)^{d+\alpha-1}}+\sum_{y \in V: \rho(z, y)>r} \frac{w_{y, z}}{\rho(y, z)^{d+\alpha}}\right)=: c_{1}\left(I_{1}(z, r)+I_{2}(z, r)\right),
\end{aligned}
$$

where in the first inequality above we have used $\left|f_{x, r}(y)-f_{x, r}(z)\right| \leqslant c_{1} r^{-1} \rho(y, z)$. According to (3.18) and (3.12), we can find a constant $R_{1} \geqslant 1$ such that for all $R_{1}<R<r_{G}, x \in B(0,2 R)$ and $R^{\theta} \leqslant r \leqslant R, \sup _{z \in B(x, r)}\left(I_{1}(z, r)+I_{2}(z, r)\right) \leqslant c_{2} r^{-\alpha}$.

Combining with all estimates above, we prove the desired assertion.

3.3. Hölder regularity. Let $\mathbb{R}_{+}:=[0, \infty)$ and $Z:=\left(Z_{t}\right)_{t \geqslant 0}=\left(U_{t}, X_{t}\right)_{t \geqslant 0}$ be the time-space process such that $U_{t}=U_{0}+t$ for any $t \geqslant 0$. Denote by $\mathbb{P}_{(s, x)}$ the probability of the process $Z$ starting from $(s, x) \in \mathbb{R}_{+} \times V$. For any subset $A \subseteq \mathbb{R}_{+} \times V$, define $\tau_{A}=\inf \left\{s>0: Z_{s} \in A\right\}$ and $\sigma_{A}=\inf \left\{s>0: Z_{s} \in A\right\}$ (for simplicity of notation we still use $\tau_{A}$ to denote the exit time from $A \subset \mathbb{R}_{+} \times V$ for process $\left.\left(Z_{t}\right)_{t \geqslant 0}\right)$. We say that a measurable function $q(t, x)$ on $\mathbb{R}_{+} \times V$ is caloric in an open set $A \subseteq \mathbb{R}_{+} \times V$, if for every relatively compact open subset $A_{1}$ of $A, q(t, x)=\mathbb{E}_{(t, x)} q\left(Z_{\tau_{A_{1}}}\right)$ for every $(t, x) \in A_{1}$.

For any $t \geqslant 0, x \in V$ and $R \geqslant 1$, let $Q(t, x, R)=\left(t, t+C_{0} R^{\alpha}\right) \times B(x, R)$ and $d \nu=d s \times d \mu$, where $C_{0}$ is the constant in (3.13). In the following, let $c_{*}$ and $\theta$ be the constants in Assumption (Exi. $(\theta))(i)$. The main result in this part is the following theorem.

Theorem 3.6. Suppose that Assumption (Exi. $(\theta)$ ) holds with some $\theta \in(0,1)$. Then, there exist constants $R_{1} \geqslant 1, \delta \in(\theta, 1), \beta \in(0,1)$ and $C_{1}>0$, independent of $R_{0}, R_{1}$ and $x_{0}$ such that for every $R_{1}<R<r_{G} /\left(2 c_{*}\right), x_{0} \in B(0, R), R^{\delta} \leqslant r \leqslant R, t_{0} \geqslant 0$ and caloric function $q$ on $Q\left(t_{0}, x_{0}, 2 r\right)$,

$$
|q(s, x)-q(t, y)| \leqslant C_{1}\|q\|_{\infty, r}\left(\frac{|t-s|^{1 / \alpha}+\rho(x, y)}{r}\right)^{\beta},
$$

holds for all $(s, x),(t, y) \in Q\left(t_{0}, x_{0}, r\right)$ such that $\left(C_{0}^{-1}|s-t|\right)^{1 / \alpha}+\rho(x, y) \geqslant 2 r^{\delta}$, where $\|q\|_{\infty, r}=$ $\sup _{(s, x) \in\left[t_{0}, t_{0}+C_{0}(2 r)^{\alpha}\right] \times V} q(s, x)$.

Remark 3.7. Note that unlike the case of random walks on the supercritical percolation cluster ([11, Proposition 3.2]), in which the Hölder regularity holds for all points in the parabolic cylinder 
when $r$ is large enough, in the preset setting we can only obtain the Hölder regularity in the region $\left(C_{0}^{-1}|s-t|\right)^{1 / \alpha}+\rho(x, y) \geqslant 2 r^{\delta}$ inside the cylinder.

To prove Theorem 3.6, we need the following Krylov-type estimates.

Proposition 3.8. If Assumption (Exi.. $(\theta))$ holds with some $\theta \in(0,1)$, then there exist constants $R_{1} \geqslant 1, \delta \in(\theta, 1)$ and $C_{1}>0$, independent of $R_{0}$ and $R_{1}$ such that for any $R_{1}<R<r_{G} /\left(2 c_{*}\right)$, $2 R^{\delta} \leqslant r \leqslant R, x \in B(0,2 R), t \geqslant 0$ and $A \subseteq Q(t, x, r / 2)$ with $\frac{\nu(A)}{\nu(Q(t, x, r / 2))} \geqslant 1 / 2$, it holds that

$$
\mathbb{P}_{(t, x)}\left(\sigma_{A}<\tau_{Q(t, x, r)}\right) \geqslant C_{1} .
$$

Proof. We write $Q_{r}=Q(t, x, r)$ for simplicity. Without loss of generality, we may and can assume that $t=0$ and $\mathbb{P}_{(0, x)}\left(\sigma_{A}<\tau_{Q_{r}}\right) \leqslant 1 / 4$; otherwise the conclusion holds trivially. Let $T=\sigma_{A} \wedge \tau_{Q_{r}}$ and $A_{s}=\{y \in V:(s, y) \in A\}$ for all $s>0$. According to the Lévy system (see [25, Appendix A]),

$$
\begin{aligned}
\mathbb{P}_{(0, x)}\left(\sigma_{A}<\tau_{Q_{r}}\right) & \geqslant \mathbb{E}_{(0, x)}\left(\sum_{s \leqslant T} \mathbb{1}_{\left\{X_{s} \neq X_{s-}, X_{s} \in A_{s}\right\}}\right)=\mathbb{E}_{(0, x)}\left[\int_{0}^{T} \sum_{u \in A_{s}} \frac{w_{X_{s}, u}}{\rho\left(X_{s}, u\right)^{d+\alpha}} \mu_{u} d s\right] \\
& \geqslant c_{M}^{-1} \mathbb{E}_{(0, x)}\left[\int_{0}^{C_{0}(r / 2)^{\alpha}} \sum_{u \in A_{s}} \frac{w_{X_{s}, u}}{\rho\left(X_{s}, u\right)^{d+\alpha}} d s ; T \geqslant C_{0}(r / 2)^{\alpha}\right] \\
& \geqslant c_{1} r^{-d-\alpha}\left(\inf _{z \in B(x, r)} \int_{0}^{C_{0}(r / 2)^{\alpha}} \sum_{u \in A_{s}} w_{z, u} d s\right) \mathbb{P}_{(0, x)}\left(T \geqslant C_{0}(r / 2)^{\alpha}\right),
\end{aligned}
$$

where in the last inequality we have used fact that $\rho(u, z) \leqslant 2 r$ for every $u, z \in B(x, r)$.

Furthermore, according to Theorem 3.3(1), there exist constants $R_{1} \geqslant 1$ and $\delta \in(\theta, 1)$ such that for any $R_{1}<R<r_{G} /\left(2 c_{*}\right), R^{\delta} \leqslant r / 2 \leqslant R$ and $x \in B(0,2 R)$,

$$
\begin{aligned}
\mathbb{P}_{(0, x)}\left(T \geqslant C_{0}(r / 2)^{\alpha}\right) & =\mathbb{P}_{(0, x)}\left(\sigma_{A} \wedge \tau_{Q_{r}} \geqslant C_{0}(r / 2)^{\alpha}\right) \\
& \geqslant 1-\mathbb{P}_{(0, x)}\left(\sigma_{A}<\tau_{Q_{r}}\right)-\mathbb{P}_{x}\left(\tau_{B(x, r)} \leqslant C_{0}(r / 2)^{\alpha}\right) \geqslant 1-\frac{1}{4}-\frac{1}{4} \geqslant \frac{1}{2},
\end{aligned}
$$

where in the first inequality we have used the fact that

$$
\mathbb{P}_{(0, x)}\left(\tau_{Q_{r}} \leqslant C_{0}(r / 2)^{\alpha}\right)=\mathbb{P}_{x}\left(\tau_{B(x, r)} \wedge\left(C_{0} r^{\alpha}\right) \leqslant C_{0}(r / 2)^{\alpha}\right)=\mathbb{P}_{x}\left(\tau_{B(x, r)} \leqslant C_{0}(r / 2)^{\alpha}\right),
$$

and the second inequality follows from (3.13).

On the other hand, let $Q_{z}^{w}(t, x, r):=\left(t, t+C_{0} r^{\alpha}\right) \times B_{z}^{w}(x, r)$. Then, for every $R_{1}<R<r_{G}$, $2 R^{\delta} \leqslant r \leqslant R, x \in B(0,2 R)$ and $z \in B(x, r)$,

$$
\begin{aligned}
\nu\left(A \cap Q_{z}^{w}(0, x, r / 2)\right) & =\int_{0}^{C_{0}(r / 2)^{\alpha}} \sum_{u \in A_{s} \cap B_{z}^{w}(x, r / 2)} \mu_{u} d s \\
& \leqslant\left(\int_{0}^{C_{0}(r / 2)^{\alpha}} \sum_{u \in A_{s} \cap B_{z}^{w}(x, r / 2)} w_{z, u}^{-1} \mu_{u} d s\right)^{1 / 2}\left(\int_{0}^{C_{0}(r / 2)^{\alpha}} \sum_{u \in A_{s}} w_{z, u} \mu_{u} d s\right)^{1 / 2} \\
& \leqslant c_{3} r^{\alpha / 2}\left(\sum_{u \in B_{z}^{w}(x, r)} w_{z, u}^{-1}\right)^{1 / 2}\left(\int_{0}^{C_{0}(r / 2)^{\alpha}} \sum_{u \in A_{s}} w_{z, u} d s\right)^{1 / 2} \\
& \leqslant c_{3} r^{\alpha / 2}\left(\sup _{z \in B(0,3 R)} \sum_{u \in B^{w}(z, 2 r)} w_{z, u}^{-1}\right)^{1 / 2}\left(\int_{0}^{C_{0}(r / 2)^{\alpha}} \sum_{u \in A_{s}} w_{z, u} d s\right)^{1 / 2} \\
& \leqslant c_{4} r^{(d+\alpha) / 2}\left(\int_{0}^{C_{0}(r / 2)^{\alpha}} \sum_{u \in A_{s}} w_{z, u} d s\right)^{1 / 2},
\end{aligned}
$$

where in the first inequality we have used the Cauchy-Schwarz inequality, the third inequality is due to the fact that $B_{z}^{w}(x, r) \subset B^{w}(z, 2 r)$ for all $z \in B(x, r)$, and the last inequality follows from (3.11). Note that, by (3.10) and the assumption that $\frac{\nu(A)}{\nu(Q(0, x, r / 2))} \geqslant 1 / 2$, we have $\nu\left(A \cap Q_{z}^{w}(0, x, r / 2)\right) \geqslant$ $\left(1 / 2+c_{0}-1\right) \cdot \nu(Q(0, x, r / 2)) \geqslant c_{5} r^{d+\alpha}$. Combining all estimates above yields that for all $R_{1}<R<$ 
$r_{G}, 2 R^{\delta} \leqslant r \leqslant R, x \in B(0,2 R)$ and $z \in B(x, r), \int_{0}^{C_{0}(r / 2)^{\alpha}} \sum_{u \in A_{s}} w_{z, u} d s \geqslant c_{6} r^{d+\alpha}$. According to all the estimates above, we prove the required assertion.

We also need the following hitting probability estimate.

Lemma 3.9. Suppose that Assumption (Exi. $(\theta)$ ) holds with some $\theta \in(0,1)$. Then there are constants $R_{1} \geqslant 1, \delta \in(\theta, 1)$ and $C_{1}>0$, independent of $R_{0}$ and $R_{1}$ such that for every $R_{1}<R<$ $r_{G} /\left(2 c_{*}\right), R^{\delta} \leqslant r \leqslant R, x \in B(0,2 R), K>4 r, t \geqslant 0$ and $z \in B(x, r / 2)$, it holds that

$$
\mathbb{P}_{x}\left(X_{\tau_{Q(t, x, r)}} \notin B(z, K)\right) \leqslant C_{1}\left(\frac{r}{K}\right)^{\alpha} .
$$

Proof. According to the Lévy system, we know that for every $z \in B(x, r / 2)$,

$$
\begin{aligned}
\mathbb{P}_{x}\left(X_{\tau_{Q(t, x, r)}} \notin B(z, K)\right) & =\mathbb{E}_{x}\left[\int_{0}^{\tau_{B(x, r)}} \sum_{y \notin B(z, K)} \frac{w_{X_{s}, y}}{\rho\left(X_{s}, y\right)^{d+\alpha}} \mu_{y} d s\right] \\
& \leqslant c_{1} \sup _{u \in B(x, r)}\left(\sum_{y \in V: \rho(u, y)>K-2 r} \frac{w_{u, y}}{\rho(u, y)^{d+\alpha}}\right) \mathbb{E}_{x}\left[\tau_{B(x, r)}\right] \\
& \leqslant c_{1} \sup _{u \in B(0,2 R)}\left(\sum_{y \in V: \rho(u, y)>K / 2} \frac{w_{u, y}}{\rho(u, y)^{d+\alpha}}\right) \mathbb{E}_{x}\left[\tau_{B(x, r)}\right] .
\end{aligned}
$$

Note that $K / 2>2 r \geqslant R^{\delta}$ and $R^{\delta} \leqslant r \leqslant R$. Then, by (3.12) and (3.15), we can find a constant $R_{1} \geqslant 1$ such that for all $R_{1}<R<r_{G} /\left(2 c_{*}\right)$ and $x \in B(0,2 R)$,

$$
\sup _{u \in B(0,2 R)}\left(\sum_{y \in V: \rho(u, y)>K / 2} \frac{w_{u, y}}{\rho(u, y)^{d+\alpha}}\right) \leqslant c_{2} K^{-\alpha}
$$

and $\mathbb{E}_{x}\left[\tau_{B(x, r)}\right] \leqslant c_{3} r^{\alpha}$. Combining with all the estimates above immediately yields (3.22).

Proof of Theorem 3.6. We mainly follow the argument of [24, Theorem 4.14] with some modification. For simplicity, we assume that $\|q\|_{\infty, r}=1$ and $q \geqslant 0$. Now, we first show that there are constants $\eta \in(0,1), \delta \in\left(\sqrt{\delta_{0}}, 1\right)$ with $\delta_{0} \in(0,1)$ being the constant $\delta$ so that all Theorem 3.3, Proposition 3.8 and Lemma 3.9 are available, $R_{1}>R_{0}$ and $\xi \in\left(0,(1 / 4) \wedge \eta^{1 / \alpha}\right.$ ) (which are determined later) such that for any $R_{1}<R<r_{G} /\left(2 c_{*}\right), R^{\delta} \leqslant r \leqslant R, k \geqslant 1$ with $\xi^{k} r \geqslant 2 r^{\delta}$, and any $(\tilde{t}, \tilde{x}) \in Q\left(t_{0}, x_{0}, r\right)$ with $x_{0} \in B(0, R)$ and $t_{0} \geqslant 0$,

$$
\sup _{Q\left(\tilde{t}, \tilde{x}, \xi^{k} r\right)} q-\inf _{Q\left(\tilde{t}, \tilde{x}, \xi^{k} r\right)} q \leqslant \eta^{k}
$$

Let $Q_{i}=Q\left(\tilde{t}, \tilde{x}, \xi^{i} r\right)$ and $B_{i}=B\left(\tilde{x}, \xi^{i} r\right)$. Define $a_{i}=\inf _{Q_{i}} q$ and $b_{i}=\sup _{Q_{i}} q$. Clearly, $b_{i}-a_{i} \leqslant \eta^{i}$ for all $i \leqslant 0$. Suppose that $b_{i}-a_{i} \leqslant \eta^{i}$ for all $i \leqslant k$ with some $k \geqslant 0$. Choose $z_{1}, z_{2} \in Q_{k+1}$ such that $q\left(z_{1}\right)=b_{k+1}$ and $q\left(z_{2}\right)=a_{k+1}$. Letting $z_{1}=\left(t_{1}, x_{1}\right)$, we define $\tilde{Q}_{k}=Q\left(t_{1}, x_{1}, \xi^{k} r\right)$, $\tilde{Q}_{k+1}=Q\left(t_{1}, x_{1}, \xi^{k+1} r\right)$ and

$$
A_{k}=\left\{z \in \tilde{Q}_{k+1}: q(z) \leqslant \frac{a_{k}+b_{k}}{2}\right\} .
$$

Without of loss of generality, we may and do assume that $\nu\left(A_{k}\right) / \nu\left(\tilde{Q}_{k+1}\right) \geqslant 1 / 2$; otherwise, we will choose $1-q$ instead of $q$. We have

$$
\begin{aligned}
b_{k+1}-a_{k+1}= & q\left(z_{1}\right)-q\left(z_{2}\right)=\mathbb{E}_{z_{1}}\left[q\left(Z_{\sigma_{A_{k}} \wedge \tau_{\tilde{Q}_{k}}}\right)\right]-q\left(z_{2}\right) \\
= & \mathbb{E}_{z_{1}}\left[q\left(Z_{\sigma_{A_{k}} \wedge \tau_{\tilde{Q}_{k}}}\right)-q\left(z_{2}\right): \sigma_{A_{k}} \leqslant \tau_{\tilde{Q}_{k}}\right] \\
& +\mathbb{E}_{z_{1}}\left[q\left(Z_{\sigma_{A_{k}} \wedge \tau_{\tilde{Q}_{k}}}\right)-q\left(z_{2}\right): \sigma_{A_{k}}>\tau_{\tilde{Q}_{k}}, X_{\tau_{\tilde{Q}_{k}}} \in B_{k-1}\right] \\
& +\sum_{i=1}^{\infty} \mathbb{E}_{z_{1}}\left[q\left(Z_{\sigma_{A_{k}} \wedge \tau_{\tilde{Q}_{k}}}\right)-q\left(z_{2}\right): \sigma_{A_{k}}>\tau_{\tilde{Q}_{k}}, X_{\tau_{\tilde{Q}_{k}}} \in B_{k-i-1} \backslash B_{k-i}\right] \\
= & : I_{1}+I_{2}+I_{3} .
\end{aligned}
$$


It is easy to see that

$$
I_{1} \leqslant\left(\frac{a_{k}+b_{k}}{2}-a_{k}\right) \mathbb{P}_{z_{1}}\left(\sigma_{A_{k}} \leqslant \tau_{\tilde{Q}_{k}}\right) \leqslant \frac{b_{k}-a_{k}}{2} p_{k} \leqslant \frac{\eta^{k}}{2} p_{k}=\eta^{k+1} \eta^{-1} \frac{p_{k}}{2}
$$

and $I_{2} \leqslant\left(b_{k-1}-a_{k-1}\right)\left(1-p_{k}\right) \leqslant \eta^{k-1}\left(1-p_{k}\right)=\eta^{k+1} \eta^{-2}\left(1-p_{k}\right)$, where $p_{k}:=\mathbb{P}_{z_{1}}\left(\sigma_{A_{k}} \leqslant \tau_{\tilde{Q}_{k}}\right)=$ $\mathbb{P}_{\left(t_{1}, x_{1}\right)}\left(\sigma_{A_{k}} \leqslant \tau_{Q\left(t_{1}, x_{1}, \xi^{k} r\right)}\right)$. On the other hand, since $\xi^{k} r \geqslant 2 r^{\delta} \geqslant 2 R^{\delta_{0}}, \tilde{x} \in B\left(x_{1}, \xi^{k+1} r\right) \subset$ $B\left(x_{1}, \xi^{k} r / 2\right)$ and $\xi^{k-i} r>4 \xi^{k} r$ for $i \geqslant 1$, we can apply (3.22) and obtain that

$$
\mathbb{P}_{x_{1}}\left(X_{\tau_{\tilde{Q}_{k}}} \in B_{k-i-1} \backslash B_{k-i}\right) \leqslant \mathbb{P}_{x_{1}}\left(X_{\tau_{Q\left(t_{1}, x_{1}, \xi^{k}\right)}} \in B_{k-i}^{c}\right) \leqslant c_{2}\left(\frac{\xi^{k} r}{\xi^{k-i} r}\right)^{\alpha}
$$

Thus,

$$
\begin{aligned}
I_{3} & \leqslant \sum_{i=1}^{\infty}\left(b_{k-i-1}-a_{k-i-1}\right) \mathbb{P}_{x_{1}}\left(X_{\tau_{\tilde{Q}_{k}}} \in B_{k-i-1} \backslash B_{k-i}\right) \\
& \leqslant c_{2} \sum_{i=1}^{\infty} \eta^{(k-i-1)}\left(\frac{\xi^{k} r}{\xi^{k-i} r}\right)^{\alpha} \leqslant \frac{c_{2} \eta^{k+1} \eta^{-2} \xi^{\alpha}}{\eta-\xi^{\alpha}}
\end{aligned}
$$

Note that, since $x_{1} \in B(0,2 R)$ and $\xi^{k} r \geqslant 2 r^{\delta} \geqslant 2 R^{\delta_{0}}$, by (3.21) we have $p_{k} \geqslant c_{3}>0$. Combining with all the conclusions above, we arrive at the statement that

$$
\begin{aligned}
b_{k+1}-a_{k+1} & \leqslant \eta^{k+1}\left(\frac{\eta^{-1} p_{k}}{2}+\eta^{-2}\left(1-p_{k}\right)+\frac{c_{2} \eta^{-2} \xi^{\alpha}}{\eta-\xi^{\alpha}}\right) \\
& =\eta^{k+1}\left[\eta^{-2}-\left(\eta^{-2}-\frac{\eta^{-1}}{2}\right) p_{k}+\frac{c_{2} \eta^{-2} \xi^{\alpha}}{\eta-\xi^{\alpha}}\right] \\
& \leqslant \eta^{k+1}\left(\eta^{-2}\left(1-c_{3}\right)+\frac{\eta^{-1} c_{3}}{2}+\frac{c_{2} \eta^{-2} \xi^{\alpha}}{\eta-\xi^{\alpha}}\right) .
\end{aligned}
$$

Choosing $\eta$ close to 1 and then $\xi \in\left(0,(1 / 4) \wedge \eta^{1 / \alpha}\right)$ close to 0 such that

$$
\eta^{-2}\left(1-c_{3}\right)+\frac{\eta^{-1} c_{3}}{2}+\frac{c_{2} \eta^{-2} \xi^{\alpha}}{\eta-\xi^{\alpha}} \leqslant 1
$$

we get $b_{k+1}-a_{k+1} \leqslant \eta_{k+1}$. This proves (3.23).

For any $(s, x),(t, y) \in Q\left(t_{0}, x_{0}, r\right)$ with $s \leqslant t$ and $\left(C_{0}^{-1}|t-s|\right)^{1 / \alpha}+\rho(x, y) \geqslant 2 r^{\delta}$, let $k$ be the smallest integer such that $\left(C_{0}^{-1}|s-t|\right)^{1 / \alpha}+\rho(x, y) \geqslant \xi^{k+1} r$. Then, $\left(C_{0}^{-1}|s-t|\right)^{1 / \alpha}+\rho(x, y) \leqslant \xi^{k} r$, and so $\xi^{k} r \geqslant 2 r^{\delta}$ and $(t, y) \in Q\left(s, x, \xi^{k} r\right)$. According to (3.23), we know that

$$
|q(s, x)-q(t, y)| \leqslant \eta^{k} \leqslant \eta^{-1}\left(\frac{\left(C_{0}^{-1}|s-t|\right)^{1 / \alpha}+\rho(x, y)}{r}\right)^{\log _{\xi} \eta} .
$$

The proof is finished.

Remark 3.10. (1) By carefully tracking the proofs of Theorems 3.3 and 3.6, we know that the constant $C_{0}$ in (3.13) and the constants $C_{1}$ in (3.14) and (3.20) only depend on $c_{M}$ and $c_{G}$ given by (2.1) and (2.2) respectively, as well as $C_{1}$ and $c_{0}$ in Assumption (Exi. $(\theta)$ ).

(2) According to Proposition 3.5, the proof of Theorem 3.3 and the arguments in this subsection, we can obtain that, when $\alpha \in(0,1)$, Theorems 3.3 and 3.6 still hold under assumption (Exi. $\left.(\theta)^{\prime}\right)$.

\section{Convergence of Stable-Like processes on metric measure spaces}

In this section, we give convergence criteria for stable-like processes on metric measure spaces. The section is split into four parts. In Subsection 4.1 we first adopt the framework from [23], which essentially assumes that the metric measure space $(F, \rho, \mu)$, as the state space of the stable-like process, is endowed with the graph approximations $\left(V_{n}, E_{V_{n}}\right)_{n \geqslant 1}$. Then, in Subsection 4.2 we define a class of Dirichlet forms $\left(D_{V_{n}}, \mathscr{F}_{V_{n}}\right)_{n \geqslant 1}$ on the approximating graphs $\left(V_{n}, E_{V_{n}}\right)_{n \geqslant 1}$, which associate a class of symmetric $\alpha$-stable-like processes $\left(X^{(n)}\right)_{n \geqslant 1}$. We also consider the corresponding scaling limit $\left(D_{0}, \mathscr{F}_{0}\right)$ of Dirichlet forms, which generates a $\alpha$-stable-like process $X$ living on $(F, \rho, \mu)$. With those constructions at hand, we will establish sufficient conditions for the weak convergence 
of $\left(X^{n}\right)_{n \geqslant 1}$ to the process $X$. The proof contains two key steps. The first one is to apply the results from [23] and get the generalized Mosco convergence of Dirichlet forms $\left(D_{V_{n}}, \mathscr{F}_{V_{n}}\right)_{n \geqslant 1}$ into $\left(D_{0}, \mathscr{F}_{0}\right)$, which indicates that the associated semigroups converge in the $L^{2}$-sense. The second one is to strengthen this convergence into the required weak convergence of $\left(X^{n}\right)_{n \geqslant 1}$. For this, we will make full use of exit time estimates for the processes $\left(X^{n}\right)_{n \geqslant 1}$ and the Hölder regularity of associated caloric functions studied in Subsections 3.2 and 3.3, respectively.

4.1. Basic setting. Let $(F, \rho, m)$ be a metric measure space, where $(F, \rho)$ is a locally compact separable and connected metric space, and $m$ is a Radon measure on $F$. For every $x \in F$ and $r>0$, let $B_{F}(x, r)=\{z \in F: \rho(z, x)<r\}$. We always assume the following assumptions on $(F, \rho, m)$.

Assumption (MMS).

(i) For any $x \in F$ and $r>0$, the closure of $B_{F}(x, r)$ is compact, and $m\left(\partial\left(B_{F}(x, r)\right)\right)=0$, where $\partial\left(B_{F}(x, r)\right)=\overline{B_{F}(x, r)} \backslash B_{F}(x, r)$.

(ii) $\rho: F \times F \rightarrow \mathbb{R}_{+}$is geodesic, i.e., for any $x, y \in F$, there exists a continuous map $\gamma$ : $[0, \rho(x, y)] \rightarrow F$ such that $\gamma(0)=x, \gamma(\rho(x, y))=y$ and $\rho(\gamma(s), \gamma(t))=t-s$ for all $0 \leqslant s \leqslant$ $t \leqslant \rho(x, y)$.

(iii) There exist constants $c_{F} \geqslant 1$ and $d>0$ such that

$$
c_{F}^{-1} r^{d} \leqslant m\left(B_{F}(x, r)\right) \leqslant c_{F} r^{d}, \quad \forall x \in F, 0<r<r_{F}:=\sup _{y, z \in F} \rho(y, z) .
$$

The metric measure space $(F, \rho, m)$ will serve as the state space of the stable-like process $Y$ which will be defined later.

According to [23, Theorem 2.1], such a metric measure space is endowed with the following graph approximations.

Lemma 4.1. Under assumption (MMS), $F$ admits a sequence of approximating graphs $\left\{G_{n}:=\right.$ $\left.\left(V_{n}, E_{V_{n}}\right), n \geqslant 1\right\}$ such that the following properties hold.

(1) For every $n \geqslant 1, V_{n} \subseteq F$, and $\left(V_{n}, E_{V_{n}}\right)$ is connected and has uniformly bounded degree. Moreover, $\cup_{n=1}^{\infty} V_{n}$ is dense in $F$.

(2) There exist positive constants $C_{1}$ and $C_{2}$ such that for every $n \geqslant 1$ and $x, y \in V_{n}$,

$$
\frac{C_{1}}{n} \rho_{n}(x, y) \leqslant \rho(x, y) \leqslant \frac{C_{2}}{n} \rho_{n}(x, y),
$$

where $\rho_{n}$ is the graph distance of $\left(V_{n}, E_{V_{n}}\right)$.

(3) For each $n \geqslant 1$, there exist a class of subsets $\left\{U_{n}(x): x \in V_{n}\right\}$ of $F$ such that $\bigcup_{x \in V_{n}} U_{n}(x) \subset$ $F, m\left(U_{n}(x) \cap U_{n}(y)\right)=0$ for $x \neq y$,

$$
V_{n} \cap \operatorname{Int} U_{n}(x)=\{x\}, \sup \left\{\rho(y, z): y, z \in U_{n}(x)\right\} \leqslant \frac{C_{3}}{n}, \quad \forall x \in V_{n},
$$

and

$$
\frac{C_{4}}{n^{d}} \leqslant m\left(U_{n}(x)\right) \leqslant \frac{C_{5}}{n^{d}}, \quad \forall n \geqslant 1, x \in V_{n},
$$

where $\operatorname{Int} U_{n}(x)$ denotes the set of the interior points of $U_{n}(x)$.

Moreover, for all $r>0$ and $y \in F$,

$$
\lim _{n \rightarrow \infty} m\left(B_{F}(y, r) \bigcap\left(F \backslash \bigcup_{x \in V_{n}} U_{n}(x)\right)\right)=0 .
$$

For each $n \geqslant 1$ and $y \in F \backslash \bigcup_{x \in V_{n}} U_{n}(x)$, there exists $z \in V_{n}$ such that $\rho(y, z) \leqslant C_{6} n^{-1}$. Here $C_{i}(i=3, \cdots, 6)$ are positive constants independent of $n$.

We will consider stable-like processes on the graphs $\left\{G_{n}\right\}_{n \geqslant 1}$. 
4.2. Stable-like processes on graphs and the metric measure spaces. We first introduce a class of Dirichlet forms $\left(D_{V_{n}}, \mathscr{F}_{V_{n}}\right)$ on the graph $\left(V_{n}, E_{V_{n}}\right)$. For any $n \geqslant 1$, define

$$
\begin{aligned}
D_{V_{n}}(f, f) & =\frac{1}{2} \sum_{x, y \in V_{n}}(f(x)-f(y))^{2} \frac{w_{x, y}^{(n)}}{\rho(x, y)^{d+\alpha}} m_{n}(x) m_{n}(y), \quad f \in \mathscr{F}_{V_{n}}, \\
\mathscr{F}_{V_{n}} & =\left\{f \in L^{2}\left(V_{n} ; m_{n}\right): D_{V_{n}}(f, f)<\infty\right\},
\end{aligned}
$$

where $\alpha \in(0,2), \rho(x, y)$ is the distance function on $F, m_{n}$ is the measure on $V_{n}$ defined by

$$
m_{n}(A):=\sum_{x \in A} m\left(U_{n}(x)\right), \quad \forall A \subset V_{n},
$$

(for simplicity, we write $m_{n}(x)=m_{n}(\{x\})$ for all $x \in V_{n}$ ), and $\left\{w_{x, y}^{(n)}: x, y \in V_{n}\right\}$ is a sequence satisfying that $w_{x, y}^{(n)} \geqslant 0$ and $w_{x, y}^{(n)}=w_{y, x}^{(n)}$ for all $x \neq y$, and

$$
\sum_{y \in V_{n}} \frac{w_{x, y}^{(n)}}{\rho(x, y)^{d+\alpha}} m_{n}(y)<\infty, \quad x \in V_{n} .
$$

We note that, in the definition of the Dirichlet form $\left(D_{V_{n}}, \mathscr{F}_{V_{n}}\right)$ we use the metric $\rho(x, y)$ instead of the graph metric $\rho_{n}(x, y)$ on $V_{n}$. According to [23, Theorem 2.1], for any $n \geqslant 1$, $\left(D_{V_{n}}, \mathscr{F}_{V_{n}}\right)$ is a regular Dirichlet form on $L^{2}\left(V_{n} ; m_{n}\right)$. Let $X^{(n)}:=\left\{\left(X_{t}^{(n)}\right)_{t \geqslant 0},\left(\mathbb{P}_{x}\right)_{x \in V_{n}}\right\}$ be the associated symmetric Markov process.

To obtain the weak convergence for $X^{(n)}$, we also introduce a kind of scaling processes associated with $\left\{X^{(n)}\right\}_{n \geqslant 1}$. For any $n \geqslant 1$, let $\mathbf{P}_{n}$ be the projection map from $\left(V_{n}, \rho\right)$ to $\left(V_{n}, \rho_{n}\right)$ such that $\mathbf{P}_{n}(x):=x$ for $x \in V_{n}$. Define a measure $\tilde{m}_{n}$ on $\left(V_{n}, \rho_{n}\right)$ as follows

$$
\tilde{m}_{n}(A)=n^{d} m_{n}\left(\mathbf{P}_{n}^{-1}(A)\right)=n^{d} \sum_{x \in \mathbf{P}_{n}^{-1}(A)} m_{n}(x), \quad A \subset V_{n} .
$$

For simplicity, $\tilde{m}_{n}(x)=\tilde{m}_{n}(\{x\})$ for any $x \in V_{n}$. For any $n \geqslant 1$, we consider the following Dirichlet form $\left(\tilde{D}_{V_{n}}, \tilde{\mathscr{F}}_{V_{n}}\right)$ on $L^{2}\left(V_{n} ; \tilde{m}_{n}\right)$ :

$$
\begin{aligned}
\tilde{D}_{V_{n}}(f, f) & =\frac{1}{2} \sum_{x, y \in V_{n}}(f(x)-f(y))^{2} \frac{\tilde{w}_{x, y}^{(n)}}{\rho_{n}(x, y)^{d+\alpha}} \tilde{m}_{n}(x) \tilde{m}_{n}(y), \quad f \in \tilde{\mathscr{F}}_{V_{n}}, \\
\tilde{\mathscr{F}}_{V_{n}} & =\left\{f \in L^{2}\left(V_{n} ; \tilde{m}_{n}\right): \tilde{D}_{V_{n}}(f, f)<\infty\right\},
\end{aligned}
$$

where

$$
\tilde{w}_{x, y}^{(n)}:=w_{x, y}^{(n)}\left(\frac{\rho_{n}(x, y)}{n \rho(x, y)}\right)^{d+\alpha}, \quad x, y \in V_{n} .
$$

Note that $\tilde{D}_{V_{n}}(f, f)=n^{d-\alpha} D_{V_{n}}(f, f)$ and $\tilde{\mathscr{F}}_{V_{n}}=\mathscr{F}_{V_{n}}$. Let $\tilde{X}^{(n)}$ be the symmetric Markov process associated with $\left(\tilde{D}_{V_{n}}, \tilde{\mathscr{F}}_{V_{n}}\right)$. According to the expressions of $\left(D_{V_{n}}, \mathscr{F}_{V_{n}}\right)$ and $\left(\tilde{D}_{V_{n}}, \tilde{\mathscr{F}}_{V_{n}}\right)$, we know that $\left(\mathbf{P}_{n}\left(X_{t}^{(n)}\right)\right)_{t \geqslant 0}$ has the same distribution as $\left(\tilde{X}_{n^{\alpha} t}^{(n)}\right)_{t \geqslant 0}$.

As a candidate of the scaling limit of the Dirichlet forms $\left(D_{V_{n}}, \mathscr{F}_{V_{n}}\right)$ on the graphs $\left(V_{n}, E_{V_{n}}\right)$, we now define a symmetric Dirichlet form $\left(D_{0}, \mathscr{F}_{0}\right)$ on $L^{2}(F ; m)$ as follows

$$
\begin{aligned}
D_{0}(f, f) & =\frac{1}{2} \int_{\{F \times F \backslash \operatorname{diag}\}}(f(x)-f(y))^{2} \frac{c(x, y)}{\rho(x, y)^{d+\alpha}} m(d x) m(d y), \quad f \in \mathscr{F}_{0}, \\
\mathscr{F}_{0} & =\left\{f \in L^{2}(F ; m): D_{0}(f, f)<\infty\right\},
\end{aligned}
$$

where $\alpha \in(0,2)$, diag $:=\{(x, y) \in F \times F: x=y\}$ and $c: F \times F \rightarrow(0, \infty)$ is a symmetric continuous function such that $0<c_{1} \leqslant c(x, y) \leqslant c_{2}<\infty$ for all $(x, y) \in F \times F \backslash$ diag and some constants $c_{1}, c_{2}$. According to (4.1) and the fact that $\alpha \in(0,2)$, we have

$$
\begin{aligned}
& \sup _{x \in F} \int_{F \backslash\{y \in F: y \neq x\}}\left(1 \wedge \rho^{2}(x, y)\right) \frac{c(x, y)}{\rho(x, y)^{d+\alpha}} m(d y) \\
& \leqslant \sup _{x \in F} \sum_{k=0}^{\infty} \int_{\left\{y \in F: 2^{-(1+k)}<\rho(y, x) \leqslant 2^{-k}\right\}} \frac{c(x, y)}{\rho(x, y)^{d+\alpha-2}} m(d y)
\end{aligned}
$$




$$
\begin{aligned}
& +\sup _{x \in F} \sum_{k=0}^{\infty} \int_{\left\{y \in F: 2^{k}<\rho(y, x) \leqslant 2^{1+k}\right\}} \frac{c(x, y)}{\rho(x, y)^{d+\alpha}} m(d y) \\
\leqslant & c_{2} \sup _{x \in F}\left(\sum_{k=0}^{\infty} m\left(B_{F}\left(x, 2^{-k}\right)\right) 2^{(d+\alpha-2)(1+k)}+\sum_{k=0}^{\infty} m\left(B_{F}\left(x, 2^{1+k}\right)\right) 2^{-(d+\alpha) k}\right) \\
\leqslant & c_{3}\left(\sum_{k=0}^{\infty} 2^{-(2-\alpha) k}+\sum_{k=0}^{\infty} 2^{-\alpha k}\right)<\infty .
\end{aligned}
$$

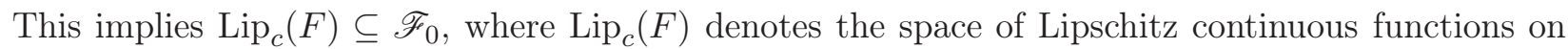
$F$ with compact support. We also need the following assumption on $\left(D_{0}, \mathscr{F}_{0}\right)$.

Assumption (Dir.) $\operatorname{Lip}_{c}(F)$ is dense in $\mathscr{F}_{0}$ under the norm $\|\cdot\|_{D_{0}, 1}:=\left(D_{0}(\cdot, \cdot)+\|\cdot\|_{L^{2}(F ; m)}^{2}\right)^{1 / 2}$. Therefore, $\left(D_{0}, \mathscr{F}_{0}\right)$ is a regular Dirichlet form on $L^{2}(F ; m)$, and there exists a strong Markov process $Y:=\left(Y_{t}\right)_{t \geqslant 0}$ associated with $\left(D_{0}, \mathscr{F}_{0}\right)$. Moreover, by [24, Theorem 1.1] or [25, Theorem 1.2], the process $Y$ has a heat kernel $p^{Y}:(0, \infty) \times F \times F \rightarrow(0, \infty)$, which is jointly continuous. In particular, the process $Y:=\left(\left(Y_{t}\right)_{t \geqslant 0},\left(\mathbb{P}_{x}^{Y}\right)_{x \in F}\right)$ can start from all $x \in F$. The process $Y$ is called a $\alpha$-stable-like process in the literature, see $[24,25]$. Two-sided estimates for heat kernel $p^{Y}(t, x, y)$ of the process $Y$ have been obtained in [24].

4.3. Generalized Mosco convergence. To study the convergence property of process $X^{(n)}$, we will use some results from [23], which are concerned with the generalized Mosco convergence of $X^{(n)}$.

For any $n \geqslant 1$, we define an extension operator $E_{n}: L^{2}\left(V_{n} ; m_{n}\right) \rightarrow L^{2}(F ; m)$ as follows

$$
E_{n}(g)(z)=\left\{\begin{array}{ll}
g(x), & z \in \operatorname{Int} U_{n}(x) \text { for some } x \in V_{n}, \\
0, & z \in F \backslash \bigcup_{x \in V_{n}} U_{n}(x),
\end{array} \quad g \in L^{2}\left(V_{n} ; m_{n}\right) .\right.
$$

Note that because $m\left(\partial U_{n}(x)\right)=0$ for any $x \in V_{n}$ by Assumption (MMS)(i), there is no need to worry about $E_{n}(g)$ on $\bigcup_{x \in V_{n}} \partial U_{n}(x)$, and the function $E_{n}(g)$ is a.s. well defined on $F$. Note also that the definition of the extension operator $E_{n}$ above is a little different from that in [23], see [23, (2.14)]. Furthermore, we define a projection (restriction) operator $\pi_{n}: L^{2}(F ; m) \rightarrow L^{2}\left(V_{n} ; m_{n}\right)$ as follows

$$
\pi_{n}(f)(x)=m_{n}(x)^{-1} \int_{U_{n}(x)} f(z) m(d z), \quad x \in V_{n}, f \in L^{2}(F ; m) .
$$

Remark 4.2. By Lemma 4.1, under assumption (MMS), the space $F$ admits a sequence of approximating graphs $\left\{\left(V_{n}, E_{V_{n}}\right): n \geqslant 1\right\}$ enjoying all the properties mentioned in Lemma 4.1. Though these properties are weaker than (AG.1)-(AG.3) in [23, Theorem 2.1], one can verify that [23, Lemma 4.1] and so [23, Theorem 4.7] still hold with notations above. These weaker properties hold for the case that $F$ is a bounded Lipschitz domain in $\mathbb{R}^{d}$.

For simplicity, we assume that there exists a point $0 \in \bigcap_{n=1}^{\infty} V_{n}$; otherwise, we can take a sequence $\left\{o_{n}\right\}_{n \geqslant 1}$ such that $o_{n} \in V_{n}$ for all $n \geqslant 1$ and $\lim _{n \rightarrow \infty} o_{n}$ exists, and then the arguments below still hold true with this limit point $0:=\lim _{n \rightarrow \infty} O_{n}$.

Fix $0 \in \cap_{n=1}^{\infty} V_{n}$. We assume that the following conditions hold for $\left\{w_{x, y}^{(n)}: x, y \in V_{n}\right\}$.

\section{Assumption (Mos.)}

(i) For every $R>0$,

$$
\lim _{\varepsilon \rightarrow 0} \limsup _{n \rightarrow \infty}\left[n^{-2 d} \sum_{x, y \in B_{F}(0, R) \cap V_{n}: 0<\rho(x, y) \leqslant \varepsilon} \frac{w_{x, y}^{(n)}}{\rho(x, y)^{d+\alpha-2}}\right]=0
$$

and

$$
\lim _{l \rightarrow \infty} \limsup _{n \rightarrow \infty}\left[n^{-2 d} \sum_{x, y \in B_{F}(0, R) \cap V_{n}: \rho(x, y) \geqslant l} \frac{w_{x, y}^{(n)}}{\rho(x, y)^{d+\alpha}}\right]=0 .
$$


(ii) For any sufficiently small $\varepsilon>0$, large $R>0$ and any $f \in \operatorname{Lip}_{c}(F)$,

$$
\lim _{n \rightarrow \infty}\left[n^{-d} \sum_{x \in B_{F}(0, R) \cap V_{n}}\left(\sum_{y \in B_{F}(0, R) \cap V_{n}: \rho(x, y)>\varepsilon}(f(x)-f(y)) \frac{\left(w_{x, y}^{(n)}-c(x, y)\right)}{\rho(x, y)^{d+\alpha}} m_{n}(y)\right)^{2}\right]=0 .
$$

(iii) For any sufficiently small $\varepsilon>0$, large $R>0$ and any $f \in C_{b}\left(B_{F}(0, R)\right)$,

$$
\lim _{n \rightarrow \infty} \sum_{x, y \in B_{F}(0, R) \cap V_{n}: \rho(x, y)>\varepsilon}(f(x)-f(y))^{2} \frac{\left(w_{x, y}^{(n)}-c(x, y)\right)}{\rho(x, y)^{d+\alpha}} m_{n}(x) m_{n}(y)=0 .
$$

Denote by $\left(P_{t}^{Y}\right)_{t \geqslant 0}$ the Markov semigroup of the process $Y$, and denote by $\left(P_{t}^{(n)}\right)_{t \geqslant 0}$ the Markov semigroup of the process $X^{(n)}$. We set $\hat{P}_{t}^{(n)} f(x)=E_{n}\left(P_{t}^{(n)}\left(\pi_{n}(f)\right)\right)(x)$ for any $f \in L^{2}(F ; m)$.

Proposition 4.3. Suppose that Assumptions (MMS), (Dir.) and (Mos.) hold. Then

$$
\lim _{n \rightarrow \infty}\left\|\hat{P}_{t}^{(n)} f-P_{t}^{Y} f\right\|_{L^{2}(F ; m)}=0, \quad f \in L^{2}(F ; m), t>0 .
$$

Proof. It is easy to see that the Dirichlet form $\left(D_{0}, \mathscr{F}_{0}\right)$ satisfies $(A 2)$ in [23, Section 2]. By assumption (Dir.) and the continuity of $c(x, y)$, we know that $(A 3)^{*}$ in $[23$, Section 2$]$ holds true.

Clearly, condition $(A 4)^{*}$ (i) in [23, Section 2] is a direct consequence of (4.8) and (4.9). For any $R, \varepsilon>0$ and $f \in \operatorname{Lip}_{c}(F)$, define

$$
\begin{aligned}
& L_{R, \varepsilon} f(x)=\int_{\left\{z \in B_{F}(0, R): \rho(z, x)>\varepsilon\right\}}(f(z)-f(x)) \frac{c(x, z)}{\rho(x, z)^{d+\alpha}} m(d z), \quad x \in F, \\
& \overline{L_{R, \varepsilon}^{n}} f(x)=\sum_{z \in B_{F}(0, R) \cap V_{n}: \rho(x, z)>\varepsilon}(f(z)-f(x)) \frac{w_{x, z}^{(n)}}{\rho(x, z)^{d+\alpha}} m_{n}(z), \quad x \in V_{n}, \\
& L_{R, \varepsilon}^{n} f(x)=E_{n}\left(\overline{L_{R, \varepsilon}^{n}} f\right)(x), \quad x \in F .
\end{aligned}
$$

Then,

$$
\int_{B_{F}(0, R)}\left|L_{R, \varepsilon}^{n} f(x)-L_{R, \varepsilon} f(x)\right|^{2} m(d x) \leqslant \sum_{i=1}^{4} I_{i, n}
$$

where

$$
\begin{aligned}
& I_{1, n}=2 \sum_{x \in B_{F}(0, R) \cap V_{n}}\left(\sum_{\substack{y \in B_{F}(0, R) \cap V_{n}: \\
\rho(x, y)>\varepsilon}}(f(x)-f(y)) \frac{\left(w_{x, y}^{(n)}-c(x, y)\right)}{\rho(x, y)^{d+\alpha}} m_{n}(y)\right)^{2} m_{n}(x), \\
& I_{2, n}=\operatorname{8osc}_{n}(f)^{2} \sum_{x \in B_{F}(0, R) \cap V_{n}}\left(\sum_{y \in B_{F}(0, R) \cap V_{n}: \rho(x, y)>\varepsilon} \frac{c(x, y)}{\rho(x, y)^{d+\alpha}} m_{n}(y)\right)^{2} m_{n}(x), \\
& I_{3, n}=8\|f\|_{\infty}^{2} \operatorname{osc}_{n}(c)^{2} \int_{B_{F}(0, R)}\left(\int_{B_{F}(0, R) \cap\{y \in F: \rho(x, y)>\varepsilon\}} \frac{1}{\rho(x, y)^{d+\alpha}} m(d y)\right)^{2} m(d x), \\
& I_{4, n}=4\|f\|_{\infty}^{2}\|c\|_{\infty}^{2} \int_{B_{F}(0, R) \cap\left(F \backslash \cup_{z \in V_{n}} U_{n}(z)\right)}\left(\int_{\substack{B_{F}(0, R) \cap\left(F \backslash \cup_{z \in \cup_{n}} U_{n}(z)\right) \\
\cap\{y \in F: \rho(x, y)>\varepsilon\}}} \frac{1}{\rho(x, y)^{d+\alpha}} m(d y)\right)^{2} m(d x), \\
& \operatorname{osc}_{n}(f)=\sup _{x \in B_{F}(0, R) \cap V_{n}, x_{1}, x_{2} \in U_{n}(x)}\left|f\left(x_{1}\right)-f\left(x_{2}\right)\right|, \\
& \operatorname{suc}_{n}(c)=\sup _{x, y \in B_{F}(0, R) \cap V_{n}, x_{1}, x_{2} \in U_{n}(x), y_{1}, y_{2} \in U_{n}(y)}\left|c\left(x_{1}, y_{1}\right)-c\left(x_{2}, y_{2}\right)\right| .
\end{aligned}
$$

It follows from (4.4) and (4.10) that $\lim _{n \rightarrow \infty} I_{1, n}=0$. Since $f \in \operatorname{Lip}_{c}(F), \operatorname{osc}_{n}(f) \rightarrow 0$ as $n \rightarrow \infty$. Then, we arrive at the statement that

$$
\limsup _{n \rightarrow \infty} I_{2, n} \leqslant c_{1} \varepsilon^{-2(d+\alpha)}\left[\limsup _{n \rightarrow \infty} \operatorname{osc}_{n}(f)^{2}\right]
$$




$$
\begin{aligned}
& \times \sup _{n \geqslant 1}\left\{n^{-3 d} \sum_{x \in B_{F}(0, R) \cap V_{n}}\left(\sum_{y \in B_{F}(0, R) \cap V_{n}: \rho(x, y)>\varepsilon} c(x, y)\right)^{2}\right\} \\
& \leqslant c_{2}(\varepsilon)\left[\limsup _{n \rightarrow \infty} \operatorname{osc}_{n}(f)^{2}\right]=0 .
\end{aligned}
$$

By the continuity of $c(x, y)$, it is also easy to see that $\lim _{n \rightarrow \infty} I_{3, n}=0$. Obviously, (4.5) implies that $\lim _{n \rightarrow \infty} I_{4, n}=0$. Therefore, we have

$$
\lim _{n \rightarrow \infty} \int_{B_{F}(0, R)}\left|L_{R, \varepsilon}^{n} f(x)-L_{R, \varepsilon} f(x)\right|^{2} m(d x)=0,
$$

which implies that condition $(A 4)^{*}$ (ii) in $[23$, Section 2] is satisfied.

Similarly, with aid of (4.11), we can claim that condition $(A 4)^{*}$ (iii) in [23, Section 2] is also fulfilled. Therefore, we can verify that all the conditions of $(A 4)^{*}$ in $[23$, Section 2] hold under assumptions (MMS), (Dir.) and (Mos.). Hence, the required assertion follows from [23, Theorem 4.7 and Theorem 8.3].

4.4. Weak convergence. The main purpose of this subsection is to establish the weak convergence theorem of the law for $X^{(n)}$ (with a fixed starting point). For any $T \in(0, \infty)$, denote by $\mathscr{D}([0, T] ; F)$ the collection of càdlàg $F$-valued functions on $[0, T]$ equipped with the Skorohod topology. Let $\mathbb{P}_{x}^{(n)}$ be the law of $X^{(n)}$ with starting point $x \in V_{n}$. Note that $\mathbb{P}_{x}^{(n)}$ can be seen as a distribution on $\mathscr{D}([0, T] ; F)$. Our approach for the weak convergence of $X^{(n)}$ contains the following two key ingredients. One is to use exit time estimates from Subsection 3.2 to show the tightness of $\mathbb{P}^{(n)}$; the other one is to apply the Hölder regularity of caloric functions from Subsection 3.3, along with Proposition 4.3, to derive the convergence of $\mathbb{P}^{(n)}$ in the sense of finite dimensional distributions.

We will make use of scaling processes $\left\{\tilde{X}^{(n)}\right\}_{n \geqslant 1}$ constructed in Subsection 4.2. First, we consider some properties of the space $\left(V_{n}, \rho_{n}, \tilde{m}_{n}\right)$. For any $x \in V_{n}$ and $r>0$, let $B_{V_{n}}(x, r)=\left\{z \in V_{n}\right.$ : $\left.\rho_{n}(z, x) \leqslant r\right\}$.

Lemma 4.4. Under assumption (MMS), there are constants $C_{0}>0$ and $c_{V} \geqslant 1$ such that for all $n \geqslant 1$,

$$
c_{V}^{-1} \leqslant \tilde{m}_{n}(x) \leqslant c_{V}, \quad x \in V_{n}
$$

and

$$
c_{V}^{-1} r^{d} \leqslant \tilde{m}_{n}\left(B_{V_{n}}(x, r)\right) \leqslant c_{V} r^{d}, \quad x \in V_{n}, 1 \leqslant r<C_{0} n r_{F},
$$

where $r_{F}$ is the constant in (4.1).

Proof. By the definition of $\tilde{m}_{n}$ and (4.4), (4.12) holds trivially.

Note that, for any $x \in V_{n}, y \in B_{F}(x, r) \cap V_{n}$ and $z \in U_{n}(y)$, by (4.3), we have $\rho(z, x) \leqslant$ $\rho(z, y)+\rho(y, x) \leqslant C_{3} n^{-1}+r$, and so $\bigcup_{y \in B_{F}(x, r) \cap V_{n}} U_{n}(y) \subseteq B_{F}\left(x, r+C_{3} n^{-1}\right)$. Hence, for any $x \in V_{n}$ and $1 \leqslant r<\left(n r_{F}-C_{3}\right) / C_{2}$ (where $C_{2}$ and $C_{3}$ are constants in (4.2) and (4.3)),

$$
\begin{aligned}
\tilde{m}_{n}\left(B_{V_{n}}(x, r)\right) & =n^{d} m_{n}\left(B_{V_{n}}(x, r) \cap V_{n}\right) \leqslant n^{d} m_{n}\left(B_{F}\left(x, C_{2} n^{-1} r\right) \cap V_{n}\right) \\
& =n^{d} \sum_{y \in B_{F}\left(x, C_{2} n^{-1} r\right) \cap V_{n}} m\left(U_{n}(y)\right) \leqslant n^{d} m\left(B_{F}\left(x, C_{2} n^{-1} r+C_{3} n^{-1}\right)\right) \leqslant c_{0} r^{d},
\end{aligned}
$$

where in the first inequality we used (4.2), the second inequality is due to the facts that $m\left(U_{n}(x) \cap\right.$ $\left.U_{n}(y)\right)=0$ for all $x \neq y$ and $\bigcup_{y \in B_{F}\left(x, C_{2} n^{-1} r\right) \cap V_{n}} U_{n}(y) \subseteq B_{F}\left(x, C_{2} n^{-1} r+C_{3} n^{-1}\right)$ as explained above, and the last inequality follows from (4.1).

On the other hand, for any $z \in B_{F}(x, r)$, by (3) in Lemma 4.1, there exists $y \in V_{n}$ such that $\rho(y, z) \leqslant c_{0} n^{-1}$ for some constant $c_{0}>0$, and so $\rho(y, x) \leqslant \rho(z, x)+\rho(z, y) \leqslant r+c_{0} n^{-1}$. This implies that $B_{F}(x, r) \subset \bigcup_{y \in B_{F}\left(x, r+c_{0} n^{-1}\right) \cap V_{n}} B_{F}\left(y, c_{0} n^{-1}\right)$. Hence, for $\left(2\left(C_{1}^{-1} c_{0}\right)\right) \vee 1<r<\left(n r_{F}+c_{0}\right) / C_{1}$ (where $C_{1}$ is the constant in (4.2)) and $x \in V_{n}$,

$$
\begin{aligned}
\tilde{m}_{n}\left(B_{V_{n}}(x, r)\right) & =n^{d} m_{n}\left(B_{V_{n}}(x, r)\right) \geqslant n^{d} m_{n}\left(B_{F}\left(x, C_{1} n^{-1} r\right) \cap V_{n}\right) \\
& =n^{d} \sum_{y \in B_{F}\left(x, C_{1} n^{-1} r\right) \cap V_{n}} m\left(U_{n}(y)\right) \geqslant c_{1} n^{d} \sum_{y \in B_{F}\left(x, C_{1} n^{-1} r\right) \cap V_{n}} m\left(B_{F}\left(y, c_{0} n^{-1}\right)\right)
\end{aligned}
$$




$$
\geqslant c_{1} n^{d} m\left(B_{F}\left(x, C_{1} n^{-1} r-c_{0} n^{-1}\right)\right) \geqslant c_{2} r^{d},
$$

where in the first inequality we used (4.2) again, the second inequality follows from (4.1) and (4.4), the third inequality is due to $\bigcup_{y \in B_{F}\left(x, C_{1} n^{-1} r\right) \cap V_{n}} B_{F}\left(y, c_{0} n^{-1}\right) \supseteq B_{F}\left(x, C_{1} n^{-1} r-c_{0} n^{-1}\right)$ as claimed before, and in the last one we have used (4.1).

Therefore, combining both estimates above and changing the corresponding constants properly, we prove (4.13).

By (4.2), for all $n \geqslant 1, \sup _{x, y \in V_{n}} \rho_{n}(x, y) \leqslant C_{1}^{-1} n r_{F}$, where $r_{F}$ is the constant in (4.1). Below, we let $C_{0}^{\prime}=C_{1}^{-1}$. For any $x, z \in V_{n}$ and $r>0$, let $B_{V_{n}, z}^{w^{(n)}}(x, r)=\left\{y \in B_{V_{n}}(x, r): w_{y, z}^{(n)}>0\right\}$, and $B_{V_{n}}^{w^{(n)}}(x, r)=B_{V_{n}, x}^{w^{(n)}}(x, r)$. We need the following further assumptions on $\left\{w_{x, y}^{(n)}: x, y \in V_{n}\right\}$.

Assumption (Wea. $(\theta)$ ). Suppose that for some fixed $\theta \in(0,1)$, there exist constants $R_{0} \geqslant 1$, $c_{0} \in(1 / 2,1)$ and $C_{3}>0$ such that the following conditions hold.

(i) For any $n \geqslant 1, R_{0}<R<C_{0}^{\prime} n r_{F}$ and $R^{\theta} / 2 \leqslant r \leqslant 2 R$,

$$
\begin{gathered}
\sup _{x \in B_{V_{n}}(0,6 R)} \sum_{y \in V_{n}: \rho_{n}(y, x) \leqslant r} \frac{w_{x, y}^{(n)}}{\rho_{n}(x, y)^{d+\alpha-2}} \leqslant C_{3} r^{2-\alpha}, \\
m_{n}\left(B_{V_{n}, z}^{w^{(n)}}(x, r)\right) \geqslant c_{0} m_{n}\left(B_{V_{n}}(x, r)\right), \quad \forall x, z \in B_{V_{n}}(0,6 R),
\end{gathered}
$$

and

$$
\sup _{x \in B_{V_{n}}(0,6 R) \cap V_{n}} \sum_{y \in V_{n}: \rho_{n}(y, x) \leqslant c_{*} r, w_{x, y}^{(n)}>0}\left(w_{x, y}^{(n)}\right)^{-1} \leqslant C_{3} r^{d},
$$

where $c_{*}=8 c_{V}^{2 / d}$ and $c_{V}$ is the constant in (4.13).

When $\alpha \in(0,1)$, (4.14) can be replaced by

$$
\sup _{x \in B_{V_{n}}(0,6 R)} \sum_{y \in V_{n}: \rho_{n}(y, x) \leqslant r} \frac{w_{x, y}^{(n)}}{\rho_{n}(x, y)^{d+\alpha-1}} \leqslant C_{3} r^{1-\alpha} .
$$

(ii) For every $n \geqslant 1, R_{0}<R<C_{0}^{\prime} n r_{F}$ and $r \geqslant R^{\theta} / 2$,

$$
\sup _{x \in B_{V_{n}}(0,6 R)} \sum_{y \in V_{n}: \rho_{n}(x, y)>r} \frac{w_{x, y}^{(n)}}{\rho_{n}(x, y)^{d+\alpha}} \leqslant C_{3} r^{-\alpha} .
$$

The main result of this section is as follows. It is in some sense a generalization of [21, Proposition 2.8]. Indeed, in our case we have the Hölder regularity of caloric functions only in the region $\left(C_{0}^{-1}|s-t|\right)^{1 / \alpha}+\rho(x, y) \geqslant 2 r^{\delta}$ (see Theorem 3.6), hence more careful arguments are required.

Theorem 4.5. Suppose that Assumptions (MMS), (Dir.), (Mos.) and (Wea.( $\theta)$ ) hold for some $\theta \in(0,1)$. Then, for any $\left\{x_{n} \in V_{n}: n \geqslant 1\right\}$ such that $\lim _{n \rightarrow \infty} x_{n}=x$ for some $x \in F$, it holds that for every $T>0, \mathbb{P}_{x_{n}}^{(n)}$ converges weakly to $\mathbb{P}_{x}^{Y}$ on the space of all probability measures on $\mathscr{D}([0, T] ; F)$, where $\mathbb{P}_{x_{n}}^{(n)}$ and $\mathbb{P}_{x}^{Y}$ denote the laws of $X^{(n)}$ and $Y$. on $\mathscr{D}([0, T] ; F)$, respectively.

Proof. Throughout the proof, we denote the law of $\left(X_{t}^{(n)}\right)_{t \geqslant 0}$ on $\mathscr{D}([0, \infty) ; F)$ and that of $\left(\tilde{X}_{t}^{(n)}\right)_{t \geqslant 0}$ on $\mathscr{D}\left([0, \infty) ; V_{n}\right)$ by $\mathbb{P}^{(n)}$ and $\tilde{\mathbb{P}}^{(n)}$, respectively. Let $X^{(n)}$ and $\tilde{X}^{(n)}$ be their associated canonical paths.

Suppose that $\left\{x_{n} \in V_{n}: n \geqslant 1\right\}$ is a sequence with $\lim _{n \rightarrow \infty} x_{n}=x$ for some $x \in F$.

Step (1): We show that for each fixed $T>0,\left\{\mathbb{P}_{x_{n}}^{(n)}\right\}_{n \geqslant 1}$ is tight on $\mathscr{D}([0, T] ; F)$. To prove the tightness of $\left\{\mathbb{P}_{x_{n}}^{(n)}\right\}_{n \geqslant 1}$, it suffices to verify that

$$
\lim _{R \rightarrow \infty} \limsup _{n \rightarrow \infty} \mathbb{P}_{x_{n}}^{(n)}\left(\sup _{s \in[0, T]} \rho\left(0, X_{s}^{(n)}\right)>R\right)=0,
$$

and for any sequence of stopping time $\left\{\tau_{n}\right\}_{n \geqslant 1}$ such that $\tau_{n} \leqslant T$ and any sequence $\left\{\varepsilon_{n}\right\}_{n \geqslant 1}$ with $\lim _{n \rightarrow \infty} \varepsilon_{n}=0$,

$$
\limsup _{n \rightarrow \infty} \mathbb{P}_{x_{n}}^{(n)}\left(\rho\left(X_{\tau_{n}+\varepsilon_{n}}^{(n)}, X_{\tau_{n}}^{(n)}\right)>\eta\right)=0, \quad \eta>0
$$


See, e.g., [1, Theorem 1].

When $r_{F}<\infty$, (4.19) holds trivially. Now, we are going to prove (4.19) for the case that $r_{F}=\infty$. As we mentioned above, $\left(\mathbf{P}_{n}\left(X_{t}^{(n)}\right)\right)_{t \geqslant 0}$ has the same distribution as $\left(\tilde{X}_{n^{\alpha} t}^{(n)}\right)_{t \geqslant 0}$, where $\left(\tilde{X}_{t}^{(n)}\right)_{t \geqslant 0}$ is a strong Markov process generated by the Dirichlet form $\left(\tilde{D}_{V_{n}}, \tilde{\mathscr{F}}_{V_{n}}\right)$. Therefore,

$$
\begin{aligned}
\mathbb{P}_{x_{n}}^{(n)}\left(\sup _{s \in[0, T]} \rho\left(X_{s}^{(n)}, 0\right)>R\right) & =\mathbb{P}_{x_{n}}^{(n)}\left(\sup _{s \in[0, T]} \rho\left(\mathbf{P}_{\mathbf{n}}\left(X_{s}^{(n)}\right), 0\right)>R\right) \\
& =\tilde{\mathbb{P}}_{x_{n}}^{(n)}\left(\sup _{s \in\left[0, n^{\alpha} T\right]} \rho\left(\tilde{X}_{s}^{(n)}, 0\right)>R\right) \\
& \leqslant \tilde{\mathbb{P}}_{x_{n}}^{(n)}\left(\sup _{s \in\left[0, n^{\alpha} T\right]} \rho_{n}\left(\tilde{X}_{s}^{(n)}, 0\right)>c_{1}^{*} n R\right),
\end{aligned}
$$

where the last inequality follows the fact that $\rho_{n}(x, y) \geqslant c_{1}^{*} n \rho(x, y)$ for all $x, y \in V_{n}$, thanks to (4.2).

On the other hand, under assumption (Wea.), it is easy to verify that assumption (Exi.) (or assumption (Exi') when $\alpha \in(0,1))$ holds for conductances $\tilde{w}_{x, y}^{(n)}$ on the space $\left(V_{n}, \rho_{n}, \tilde{m}_{n}\right)$ with associated constants independent of $n$. Combining this fact with (4.12) and (4.13), we can apply Theorem 3.3 and Remark 3.10(1) (or Remark 3.10(2)) to derive that for any fixed $\theta^{\prime} \in(\theta, 1)$, there exist constants $\delta \in(\theta, 1)$ and $R_{1} \geqslant 1$, such that for all $n \geqslant 1, R_{1}<R<C_{0}^{\prime} r_{F} n$ and $R^{\delta} \leqslant r \leqslant R$,

$$
\sup _{x \in B_{V_{n}}(0,2 R) \cap V_{n}} \tilde{\mathbb{P}}_{x}^{(n)}\left(\tau_{B_{V_{n}}(0, r)}\left(\tilde{X}^{(n)}\right) \leqslant t\right) \leqslant c_{1}\left(\frac{t}{r^{\alpha}}\right)^{1 / 3}, \quad \forall t \geqslant r^{\theta^{\prime} \alpha},
$$

where $B_{V_{n}}(x, r)=\left\{z \in V_{n}: \rho_{n}(z, x) \leqslant r\right\}, \tau_{B_{V_{n}}(0, r)}\left(\tilde{X}^{(n)}\right)$ is the first exit time from $B_{V_{n}}(0, r)$ of the process $\tilde{X}^{(n)}$, and $c_{1}>0$ is independent of $R_{1}, n, r, R$ and $r_{F}$.

Suppose that $\rho\left(x_{n}, 0\right) \leqslant K$ for all $n \geqslant 1$ and some constant $K>0$. Note that, also thanks to $(4.2), \rho_{n}\left(x_{n}, 0\right) \leqslant c_{2}^{*} n \rho\left(x_{n}, 0\right) \leqslant c_{2}^{*} n K$. For every fixed $R>2 c_{2}^{*} K / c_{1}^{*}$ and $T>0$, we have $R_{1}<c_{1}^{*} n R<C_{0}^{\prime} n r_{F}$ (since $\left.r_{F}=\infty\right)$ and $n^{\alpha} T>\left(c_{1}^{*} n R / 2\right)^{\theta^{\prime} \alpha}$ for $n$ large enough. Thus, by (4.21) and (4.22),

$$
\begin{aligned}
\mathbb{P}_{x_{n}}^{(n)}\left(\sup _{s \in[0, T]} \rho\left(X_{s}^{(n)}, 0\right)>R\right) & \leqslant \tilde{\mathbb{P}}_{x_{n}}^{(n)}\left(\sup _{s \in\left[0, n^{\alpha} T\right]} \rho_{n}\left(\tilde{X}_{s}^{(n)}, 0\right)>c_{1}^{*} n R\right) \\
& \leqslant \sup _{z \in B_{V_{n}}\left(0, c_{2}^{*} n K\right) \cap V_{n}} \tilde{\mathbb{P}}_{z}^{(n)}\left(\tau_{B_{V_{n}}\left(0, c_{1}^{*} n R\right)}\left(\tilde{X}^{(n)}\right) \leqslant n^{\alpha} T\right) \\
& \leqslant \sup _{z \in B_{V_{n}}\left(0, c_{1}^{*} n R / 2\right) \cap V_{n}} \tilde{\mathbb{P}}_{z}^{(n)}\left(\tau_{B_{V_{n}}\left(z, c_{1}^{*} n R / 2\right)}\left(\tilde{X}^{(n)}\right) \leqslant n^{\alpha} T\right) \\
& \leqslant c_{1}\left(\frac{n^{\alpha} T}{\left(c_{1}^{*} n R / 2\right)^{\alpha}}\right)^{1 / 3}=c_{2}\left(\frac{T}{R^{\alpha}}\right)^{1 / 3},
\end{aligned}
$$

which implies

$$
\lim _{R \rightarrow \infty} \limsup _{n \rightarrow \infty} \mathbb{P}_{x_{n}}^{(n)}\left(\sup _{s \in[0, T]} \rho\left(X_{s}^{(n)}, 0\right)>R\right) \leqslant \lim _{R \rightarrow \infty} c_{2}\left(\frac{T}{R^{\alpha}}\right)^{1 / 3}=0 .
$$

This proves (4.19).

Next, let $\left\{\tau_{n}\right\}_{n \geqslant 1}$ be a sequence of stopping time such that $\tau_{n} \leqslant T$, and $\left\{\varepsilon_{n}\right\}_{n \geqslant 1}$ be a sequence such that $\lim _{n \rightarrow \infty} \varepsilon_{n}=0$. By the strong Markov property, for every $\eta>0$ small enough and $R \geqslant 1$ large enough,

$$
\begin{aligned}
& \left.\mathbb{P}_{x_{n}}^{(n)}\left(\rho\left(X_{\tau_{n}+\varepsilon_{n}}^{(n)}, X_{\tau_{n}}^{(n)}\right)>\eta\right)=\mathbb{E}_{x_{n}}^{(n)}\left[\mathbb{P}_{X_{\tau_{n}}^{(n)}}^{(n)} \rho\left(X_{\varepsilon_{n}}^{(n)}, X_{0}^{(n)}\right)>\eta\right)\right] \\
& \leqslant \sup _{z \in B_{F}(0, R) \cap V_{n}} \mathbb{P}_{z}^{(n)}\left(\rho\left(X_{\varepsilon_{n}}^{(n)}, X_{0}^{(n)}\right)>\eta\right)+\mathbb{P}_{x_{n}}^{(n)}\left(\sup _{s \in[0, T]} \rho\left(X_{s}^{(n)}, 0\right)>R\right) \\
& \leqslant \sup _{z \in B_{V_{n}}\left(0,\left(c_{2}^{*} n R\right) \wedge\left(C_{0}^{\prime} n r_{F}\right)\right) \cap V_{n}} \tilde{\mathbb{P}}_{z}^{(n)}\left(\rho_{n}\left(\tilde{X}_{n^{\alpha} \varepsilon_{n}}^{(n)}, \tilde{X}_{0}^{(n)}\right)>c_{1}^{*} n \eta\right)+\mathbb{P}_{x_{n}}^{(n)}\left(\sup _{s \in[0, T]} \rho\left(X_{s}^{(n)}, 0\right)>R\right) \\
& \leqslant \tilde{\mathbb{P}}_{z \in B_{V_{n}}\left(0,\left(c_{2}^{*} n R\right) \wedge\left(C_{0}^{\prime} n r_{F}\right)\right) \cap V_{n}} \tilde{\mathbb{P}}_{z}^{(n)}\left(\tau_{B_{V_{n}}\left(z, c_{1}^{*} n \eta\right)}\left(\tilde{X}^{(n)}\right) \leqslant n^{\alpha} \varepsilon_{n}\right)+\mathbb{P}_{x_{n}}^{(n)}\left(\sup _{s \in[0, T]} \rho\left(X_{s}^{(n)}, 0\right)>R\right),
\end{aligned}
$$


where in the second inequality we have used the fact that $c_{1}^{*} n \rho(x, y) \leqslant \rho_{n}(x, y) \leqslant c_{2}^{*} n \rho(x, y)$ for $x, y \in V_{n}$, due to (4.2). Taking $n$ large enough and $\eta$ small enough such that $c_{2}^{*} n R>R_{1}$ and $\left(c_{2}^{*} n R\right)^{\delta} \leqslant c_{1}^{*} n \eta \leqslant\left(c_{2}^{*} n R\right) \wedge\left(C_{0}^{\prime} n r_{F}\right)$. Then, it follows from (4.22) that

$$
\begin{aligned}
& \sup _{z \in B_{V_{n}}\left(0,\left(c_{2}^{*} n R\right) \wedge\left(C_{0}^{\prime} n r_{F}\right)\right) \cap V_{n}} \tilde{\mathbb{P}}_{z}^{(n)}\left(\tau_{B_{V_{n}}\left(z, c_{1}^{*} n \eta\right)}\left(\tilde{X}^{(n)}\right) \leqslant n^{\alpha} \varepsilon_{n}\right) \\
& \leqslant \sup _{z \in B_{V_{n}}\left(0,\left(c_{2}^{*} n R\right) \wedge\left(C_{0}^{\prime} n r_{F}\right)\right) \cap V_{n}} \tilde{\mathbb{P}}_{z}^{(n)}\left(\tau_{B_{V_{n}}\left(z, c_{1}^{*} n \eta\right)}\left(\tilde{X}^{(n)}\right) \leqslant\left(n^{\alpha} \varepsilon_{n}\right) \vee\left(2 c_{1}^{*} n \eta\right)^{\theta^{\prime} \alpha}\right) \\
& \leqslant c_{1}\left(\frac{\left(n^{\alpha} \varepsilon_{n}\right) \vee\left(2 c_{1}^{*} n \eta\right)^{\theta^{\prime} \alpha}}{\left(c_{1}^{*} n \eta\right)^{\alpha}}\right)^{1 / 3} \leqslant c_{3}\left(\left(\varepsilon_{n} \eta^{-\alpha}\right) \vee(n \eta)^{-\left(1-\theta^{\prime}\right) \alpha}\right)^{1 / 3} .
\end{aligned}
$$

Combining both estimates above with (4.19), we obtain (4.20).

Step (2): Now it suffices to show that any finite dimensional distribution of $\mathbb{P}_{x_{n}}^{(n)}$ converges to that of $\mathbb{P}_{x}^{Y}$. We first claim that for any fixed $t>0, f \in C_{\infty}(F) \cap L^{2}(F ; m)$ and a sequence $\left\{z_{n}: z_{n} \in V_{n}\right\}_{n=1}^{\infty}$ with $\lim _{n \rightarrow \infty} z_{n}=z \in F$,

$$
\lim _{n \rightarrow \infty} E_{n}\left(P_{t}^{(n)} f\right)\left(z_{n}\right)=P_{t}^{Y} f(z)
$$

where $C_{\infty}(F)$ denotes the set of continuous functions on $F$ vanishing at infinity.

Indeed, according to assumption (Mos.), Proposition 4.3 and (4.5), there are a subsequence of $\left\{\hat{P}_{t}^{(n)} f: n \geqslant 1\right\}$ (we still denote it by $\left\{\hat{P}_{t}^{(n)} f: n \geqslant 1\right\}$ for simplicity) and a sequence $\left\{y_{k} \in\right.$ $\left.\cap_{n \geqslant N_{0}} \cup_{x \in V_{n}} \operatorname{Int}\left(U_{n}(x)\right): k \geqslant 1\right\}$ with some $N_{0} \geqslant 1$ such that (i) $y_{k} \neq z$ and $\lim _{k \rightarrow \infty} y_{k}=z$; (ii) for every $k \geqslant 1$,

$$
\lim _{n \rightarrow \infty} \hat{P}_{t}^{(n)} f\left(y_{k}\right)=P_{t}^{Y} f\left(y_{k}\right)
$$

For every $k \geqslant 1$ and $t>0$, we have

$$
\begin{aligned}
& \left|E_{n}\left(P_{t}^{(n)} f\right)\left(z_{n}\right)-P_{t}^{Y} f(z)\right| \\
& \leqslant\left|\hat{P}_{t}^{(n)} f\left(y_{k}\right)-P_{t}^{Y} f\left(y_{k}\right)\right|+\left|\hat{P}_{t}^{(n)} f\left(y_{k}\right)-E_{n}\left(P_{t}^{(n)} f\right)\left(y_{k}\right)\right| \\
& \quad+\left|E_{n}\left(P_{t}^{(n)} f\right)\left(y_{k}\right)-E_{n}\left(P_{t}^{(n)} f\right)\left(z_{n}\right)\right|+\left|P_{t}^{Y} f(z)-P_{t}^{Y} f\left(y_{k}\right)\right| \\
& =:\left|\hat{P}_{t}^{(n)} f\left(y_{k}\right)-P_{t}^{Y} f\left(y_{k}\right)\right|+\sum_{i=1}^{3} J_{i, n, k} .
\end{aligned}
$$

Recall that $\hat{P}_{t}^{(n)} f(x)=E_{n}\left(P_{t}^{(n)}\left(\pi_{n}(f)\right)\right)(x)$ for all $x \in F$. By the definition of $\pi_{n}$,

$$
\lim _{n \rightarrow \infty} \sup _{z \in V_{n}}\left|\pi_{n}(f)(z)-f(z)\right|=0
$$

for any $f \in C_{\infty}(F)$. Hence,

$$
\begin{aligned}
\lim _{n \rightarrow \infty} \sup _{k \geqslant 1} J_{1, n, k} & =\lim _{n \rightarrow \infty} \sup _{k \geqslant 1}\left|E_{n}\left(P_{t}^{(n)}\left(\pi_{n}(f)\right)\right)\left(y_{k}\right)-E_{n}\left(P_{t}^{(n)} f\right)\left(y_{k}\right)\right| \\
& \leqslant \lim _{n \rightarrow \infty} \sup _{z \in V_{n}}\left|\pi_{n} f(z)-f(z)\right|=0
\end{aligned}
$$

where in the last inequality we used the contractivity of $\left(P_{t}^{(n)}\right)_{t \geqslant 1}$ in $L^{\infty}\left(V_{n} ; m_{n}\right)$.

In the following, for any $x \in \cap_{n} \geqslant N_{0} \cup_{x \in V_{n}} \operatorname{Int}\left(U_{n}(x)\right)$ and $n \geqslant N_{0}$, let $[x]_{n} \in V_{n}$ be such that $x \in U_{n}\left([x]_{n}\right)$ and $\rho\left(x,[x]_{n}\right) \leqslant c_{3}^{*} n^{-1}$, due to (3) in Lemma 4.1. For any $n \geqslant N_{0}$ and $z \in \cap_{n} \geqslant N_{0} \cup_{x \in V_{n}}$ $\operatorname{Int}\left(U_{n}(x)\right)$, noticing that $\left(\tilde{X}_{n^{\alpha} t}^{(n)}\right)_{t \geqslant 0}$ has the same distribution as $\left(\mathbf{P}_{\mathbf{n}}\left(X_{t}^{(n)}\right)\right)_{t \geqslant 0}$,

$$
E_{n}\left(P_{t}^{(n)} f\right)(z)=P_{t}^{(n)} f\left([z]_{n}\right)=\mathbb{E}_{[z]_{n}}^{(n)}\left[f\left(X_{t}^{(n)}\right)\right]=\tilde{\mathbb{E}}_{[z]_{n}}^{(n)}\left[f\left(\tilde{X}_{n^{\alpha} t}^{(n)}\right)\right]=\tilde{P}_{n^{\alpha} t}^{(n)} f\left([z]_{n}\right),
$$

where $\tilde{P}_{t}^{(n)} f(\cdot):=\tilde{\mathbb{E}}^{(n)}\left[f\left(\tilde{X}_{t}^{(n)}\right)\right]$ is the Markov semigroup of $\tilde{X}^{(n)}:=\left(\tilde{X}_{t}^{(n)}\right)_{t \geqslant 0}$. As mentioned above, due to assumption (Wea. $(\theta)$ ) and Lemma 4.4, we can apply Theorem 3.6 and Remark 3.10(1) (also thanks to Remark 3.10(2)) to obtain that there are constants $\delta \in(\theta, 1)$ and $R_{1} \geqslant 1$ such that for all $R_{1}<R<C_{0}^{\prime} n r_{F},(3.20)$ holds for every $\left(\tilde{X}_{t}^{(n)}\right)_{t \geqslant 0}$ and with associated constants independent of 
$n$. Let $C_{0}>0$ be the constant in (3.13). For fixed $T>0$, we define $H_{T, n, f}(s, x)=\tilde{P}_{1+n^{\alpha} T-s}^{(n)} f(x)$, which is a caloric function on $Q_{V_{n}}\left(0, y,\left(2^{-1} C_{0}^{-1} n^{\alpha} T\right)^{1 / \alpha}\right)$ for each $y \in V_{n}$. Take $K$ large enough such that $K>\left(2^{-1} C_{0}^{-1} t\right)^{1 / \alpha}, R_{1}<n K<C_{0}^{\prime} n r_{F}$ and $z_{n} \in B_{V_{n}}(0, n K)$ for all $n \geqslant 1$. According the facts that $y_{k} \rightarrow z$ as $k \rightarrow \infty$ and $y_{k} \neq z$ for all $k \geqslant 1$, for any fixed $t>0$, every $k \geqslant 1$ and $n \geqslant N_{0}$ large enough, it holds that $0<\varepsilon_{k}<\rho\left(y_{k}, z_{n}\right) \leqslant \tilde{r}_{0} \leqslant\left(4 c_{2}^{*}\right)^{-1}\left(\left(2^{-1} C_{0}^{-1} t\right)^{1 / \alpha} \wedge\left(2^{-1} C_{0}^{\prime} r_{F}\right)\right)$, where $\tilde{r}_{0}$ and $\varepsilon_{k}$ are positive constants with $\lim _{k \rightarrow \infty} \varepsilon_{k}=0$, and $c_{2}^{*}>0$ is the constant such that $\rho_{n}(x, y) \leqslant c_{2}^{*} n \rho(x, y)$ for any $x, y \in V_{n}$. Furthermore, for these fixed $k$ and $t$, we take $n$ large enough such that $(n K)^{\delta} \leqslant r_{n} \leqslant n K$ and $n \varepsilon_{k} \geqslant 4\left(c_{1}^{*}\right)^{-1} r_{n}^{\delta}$, where $r_{n}:=\left(4 c_{2}^{*}\right)^{-1} n \tilde{r}_{0}$. Hence,

$$
\begin{aligned}
\rho_{n}\left(z_{n},\left[y_{k}\right]_{n}\right) & \geqslant c_{1}^{*} n \rho\left(z_{n},\left[y_{k}\right]_{n}\right) \geqslant c_{1}^{*} n\left(\rho\left(z_{n}, y_{k}\right)-\rho\left(y_{k},\left[y_{k}\right]_{n}\right)\right) \\
& \geqslant c_{1}^{*} n \varepsilon_{k}-2 c_{1}^{*} c_{3}^{*} \geqslant r_{n}^{\delta}
\end{aligned}
$$

and

$$
\begin{aligned}
\rho_{n}\left(z_{n},\left[y_{k}\right]_{n}\right) & \leqslant c_{2}^{*} n \rho\left(z_{n},\left[y_{k}\right]_{n}\right) \leqslant c_{2}^{*} n\left(\rho\left(z_{n}, y_{k}\right)+\rho\left(y_{k},\left[y_{k}\right]_{n}\right)\right) \\
& \leqslant 4^{-1} r_{n}+2 c_{2}^{*} c_{3}^{*} \leqslant 2^{-1} r_{n},
\end{aligned}
$$

where we used the fact that $\rho\left(y_{k},\left[y_{k}\right]_{n}\right) \leqslant c_{3}^{*} n^{-1}$ for all $k$.

Then as a summary, $(n K)^{\delta} \leqslant r_{n} \leqslant n K, z_{n} \in B_{V_{n}}(0, n K)$, and $z_{n},\left[y_{k}\right]_{n} \in Q_{V_{n}}\left(0, z_{n}, r_{n}\right)$ with $\rho_{n}\left(z_{n},\left[y_{k}\right]_{n}\right) \geqslant r_{n}^{\delta}$. Now, applying (3.20) to the caloric function $H_{t, n, f}$ on $Q_{V_{n}}\left(0, z_{n}, r_{n}\right)$, we can obtain that

$$
\begin{aligned}
& \left|\tilde{P}_{n^{\alpha} t}^{(n)} f\left(\left[y_{k}\right]_{n}\right)-\tilde{P}_{n^{\alpha} t}^{(n)} f\left(z_{n}\right)\right| \\
& =\left|H_{t, n, f}\left(1,\left[y_{k}\right]_{n}\right)-H_{t, n, f}\left(1, z_{n}\right)\right| \leqslant c_{4}\left\|\tilde{P}_{n^{\alpha} t}^{(n)} f\right\|_{\infty}\left|\frac{\rho_{n}\left(\left[y_{k}\right]_{n}, z_{n}\right)}{r_{n}}\right|^{\beta} \\
& \leqslant c_{5}(t)\|f\|_{\infty} \rho\left(\left[y_{k}\right]_{n}, z_{n}\right)^{\beta} \leqslant c_{6}(t)\|f\|_{\infty}\left(\rho\left(y_{k}, z_{n}\right)^{\beta}+n^{-\beta}\right) .
\end{aligned}
$$

In particular, constants $c_{4}, c_{5}(t)$ and $c_{6}(t)$ are independent of $n$, since (3.20) holds for $\left(\tilde{X}_{t}^{(n)}\right)_{t \geqslant 0}$ with associated constants independent of $n$.

This yields immediately that

$$
\begin{aligned}
\limsup _{n \rightarrow \infty} J_{2, n, k} & =\limsup _{n \rightarrow \infty}\left|\tilde{P}_{n^{\alpha} t}^{(n)} f\left(\left[y_{k}\right]_{n}\right)-\tilde{P}_{n^{\alpha} t}^{(n)} f\left(z_{n}\right)\right| \\
& \leqslant c_{6}(t) \limsup _{n \rightarrow \infty}\|f\|_{\infty}\left(\rho\left(y_{k}, z_{n}\right)^{\beta}+n^{-\beta}\right)=c_{7}(t)\|f\|_{\infty} \rho\left(y_{k}, z\right)^{\beta} .
\end{aligned}
$$

According to [24, Theorem 4.14], $J_{3, n, k} \leqslant c_{8}(t)\|f\|_{\infty} \rho\left(y_{k}, z\right)^{\beta}$.

Combining all estimates with (4.25) and (4.24), we arrive at the statement that

$$
\limsup _{n \rightarrow \infty}\left|E_{n}\left(P_{t}^{(n)} f\right)\left(z_{n}\right)-P_{t}^{Y} f(z)\right| \leqslant c_{9}(t)\|f\|_{\infty} \rho\left(y_{k}, z\right)^{\beta},
$$

where $c_{9}(t)>0$ is independent of $k$. Note that $k$ is arbitrary, letting $k \rightarrow \infty$ in the last inequality, then we prove (4.23). In particular, according to [21, Lemma 2.7], (4.23) implies that for every compact set $K \subseteq F$,

$$
\limsup _{n \rightarrow \infty} \sup _{x \in K}\left|E_{n}\left(P_{t}^{(n)} f\right)(x)-P_{t}^{Y} f(x)\right|=0 .
$$

Next, for any $f_{1}, f_{2} \in C_{\infty}(F), 0<s<t \leqslant T$ and any sequence $x_{n} \in V_{n}$ with $\lim _{n \rightarrow \infty} x_{n}=x \in F$,

$$
\begin{aligned}
& \mathbb{E}_{x_{n}}^{(n)}\left[f_{1}\left(X_{s}^{(n)}\right) f_{2}\left(X_{t}^{(n)}\right)\right]=\mathbb{E}_{x_{n}}^{(n)}\left[f_{1}\left(X_{s}^{(n)}\right) P_{t-s}^{(n)} f_{2}\left(X_{s}^{(n)}\right)\right] \\
& =\mathbb{E}_{x_{n}}^{(n)}\left[f_{1}\left(X_{s}^{(n)}\right) P_{t-s}^{Y} f_{2}\left(X_{s}^{(n)}\right)\right]+\mathbb{E}_{x_{n}}^{(n)}\left[f_{1}\left(X_{s}^{(n)}\right)\left(P_{t-s}^{(n)} f_{2}\left(X_{s}^{(n)}\right)-P_{t-s}^{Y} f_{2}\left(X_{s}^{(n)}\right)\right)\right] \\
& =: J_{1, n}+J_{2, n} .
\end{aligned}
$$

Set $g(z)=f_{1}(z) P_{t-s}^{Y} f_{2}(z)$. Then $g \in C_{\infty}(F)$, due to the $C_{\infty}$-Feller property of the process $Y$, see [24, Theorem 1.1]. Then, according to (4.23), we have

$$
\lim _{n \rightarrow \infty} J_{1, n}=\lim _{n \rightarrow \infty} P_{t}^{(n)} g\left(x_{n}\right)=P_{s}^{Y} g(x)=\mathbb{E}_{x}^{Y}\left[f_{1}\left(Y_{s}\right) f_{2}\left(Y_{t}\right)\right] .
$$


On the other hand, for any $t>0, R>2 K$ and $n$ large enough,

$$
J_{2, n} \leqslant\left\|f_{1}\right\|_{\infty} \sup _{z \in B_{F}(0, R)}\left|E_{n}\left(P_{t-s}^{(n)} f_{2}\right)(z)-P_{t-s}^{Y} f_{2}(z)\right|+\left\|f_{1}\right\|_{\infty}\left\|f_{2}\right\|_{\infty} \mathbb{P}_{x_{n}}^{(n)}\left(\sup _{s \in[0, t]} \rho\left(X_{s}^{(n)}, 0\right)>R\right),
$$

By (4.19) and (4.26), we let $n \rightarrow \infty$ and then $R \rightarrow \infty$ in the last inequality, yielding that $\lim _{n \rightarrow \infty} J_{2, n}=0$. Combining all above estimates, we prove that

$$
\lim _{n \rightarrow \infty} \mathbb{E}_{x_{n}}^{(n)}\left[f_{1}\left(X_{s}^{(n)}\right) f_{2}\left(X_{t}^{(n)}\right)\right]=\mathbb{E}_{x}^{Y}\left[f_{1}\left(Y_{s}\right) f_{2}\left(Y_{t}\right)\right] .
$$

Following the same arguments as above and using the induction procedure, we can obtain from [31, Chapter 3; Proposition 4.4 and Theorem 7.8(b)] that any finite dimensional distribution of $\mathbb{P}_{x_{n}}^{(n)}$ converges to $\mathbb{P}_{x}^{Y}$. The proof is finished.

Remark 4.6. As shown in the proof of Theorem 4.5 above, the role of adopting the generalized Mosco convergence is to identify the limit process in the $L^{2}$ sense. Actually, according to [23, Theorem 5.1], under Assumption (Mos.) only, any finite dimensional distribution of $X^{(n)}$ converges to that of $Y$, when the initial distribution is absolutely continuous with respect to the reference measure $m$. Thus, Theorem 4.5 improves this weak convergence for any initial distribution. We emphasize that such improvement is highly non-trivial, see [33] for discussions on the uniformly elliptic case by using heat kernel estimates. Here, we will make use of the Hölder regularity of caloric functions on large scale (Theorem 3.6). This is much weaker than the approach used in [21, Proposition 2.8], where the Hölder regularity of caloric functions is assumed to be satisfied on the whole space.

\section{RANdom CONduCtance model: Quenched inVARIANCE PRINCIPLE}

In this section, we will apply results from Section 4 to study the quenched invariance principle for a few random conductance models with stable-like jumps.

5.1. Quenched invariance principle for stable-like processes on $d$-sets. Let $(F, \rho, m)$ be a metric measure space satisfying assumption (MMS). By Lemma 4.1, we have a sequence of graphs with measure $\left\{\left(V_{n}, \rho_{n}, m_{n}\right): n \geqslant 1\right\}$ that approximate $(F, \rho, m)$. In this part, we further assume the following:

(i) $\rho(\cdot, \cdot)$ is a metric with dilation; namely, there exists another distance $\bar{\rho}$ on $F$ such that

(i') for all $x, y \in F, C_{1} \bar{\rho}(x, y) \leqslant \rho(x, y) \leqslant C_{2} \bar{\rho}(x, y)$ holds for some constants $0<C_{1}<$ $C_{2}<\infty$.

$\left(\mathrm{i}^{\prime \prime}\right)$ for each $x, y \in V_{1}$ and $n \in \mathbb{N}$, there exist $x^{\left(n^{-1}\right)}$ and $y^{\left(n^{-1}\right)} \in V_{n}$ (we will write $x^{\left(n^{-1}\right)}:=$ $n^{-1} x, y^{\left(n^{-1}\right)}:=n^{-1} y$ for notational simplicity) such that $\bar{\rho}\left(n^{-1} x, n^{-1} y\right)=n^{-1} \bar{\rho}(x, y)$.

(ii) There exists $0 \in V_{1} \subset F$ such that $n^{-1} 0=0$ for all $n \in \mathbb{N}$.

(iii) $V_{n}=n^{-1} V_{1}:=\left\{n^{-1} z: z \in V_{1}\right\}$, and $F$ is a closure of $\cup_{n \geqslant 1} V_{n}$. Moreover, $m_{n}=n^{-d} \mu_{n}$, $\mu_{n}(A)=\mu_{1}(n A)$ for all $A \subset V_{n}$ and $n \in \mathbb{N}$, where $\mu_{n}$ denotes the counting measure on $V_{n}$.

Remark 5.1. Obviously conditions $\left(\mathrm{i}^{\prime}\right)$ and $\left(\mathrm{i}^{\prime \prime}\right)$ in assumption (i) above hold true for a bounded Lipschitz domain $F \subset \mathbb{R}^{d}$. For simplicity, in the arguments below we assume that $\rho\left(n^{-1} x, n^{-1} y\right)$ $=n^{-1} \rho(x, y)$ for all $n \in \mathbb{N}$ and $x, y \in V_{1}$; otherwise, we can express Dirichlet forms $\left(D_{V_{n}}^{\omega}, \mathscr{F}_{n}^{\omega}\right)$ and $\left(D_{0}, \mathscr{F}_{0}\right)$ below with $\rho, w_{x, y}^{(n)}(\omega)$ and $c(x, y)$ replaced by $\bar{\rho}, \bar{w}_{x, y}^{(n)}(\omega):=\frac{\bar{\rho}(x, y)^{d+\alpha}}{\rho(x, y)^{d+\alpha}} w_{x, y}^{(n)}(\omega)$ and $\bar{c}(x, y):=\frac{\bar{\rho}(x, y)^{d+\alpha}}{\rho(x, y)^{d+\alpha}} c(x, y)$, respectively. Hence, by applying the arguments below for $\bar{\rho}, \bar{w}_{x, y}^{(n)}(\omega)$ and $\bar{c}(x, y)$, we can still obtain the quenched invariance principle for $\left(X_{t}^{\omega}\right)_{t \geqslant 0}$.

Let $\left\{w_{x, y}(\omega): x, y \in V_{1}\right\}$ be a sequence of random variables defined on a probability space $(\Omega, \mathscr{F}, \mathbb{P})$ such that $w_{x, y}(\omega)=w_{y, x}(\omega)$ and $w_{x, y}(\omega) \geqslant 0$ for all $x \neq y \in V_{1}$. For any $x \in V_{n}$, $m_{n}(x):=m_{n}(\{x\})=n^{-d}$. Define

$$
w_{x, y}^{(n)}(\omega)=w_{n x, n y}(\omega)
$$

We consider the following class of Dirichlet forms

$$
D_{V_{n}}^{\omega}(f, f)=\frac{1}{2} \sum_{x, y \in V_{n}}(f(x)-f(y))^{2} \frac{w_{x, y}^{(n)}(\omega)}{\rho(x, y)^{d+\alpha}} m_{n}(x) m_{n}(y), \quad f \in \mathscr{F}_{n}^{\omega},
$$




$$
\mathscr{F}_{n}^{\omega}=\left\{f \in L^{2}\left(V_{n} ; m_{n}\right): D_{V_{n}}^{\omega}(f, f)<\infty\right\} .
$$

Let $X^{V_{1}, \omega}$ be the strong Markov process on $V_{1}$ associated with $\left(D_{V_{1}}^{\omega}, \mathscr{F}_{1}^{\omega}\right)$. Then, it is easy to show that for a.s. $\omega \in \Omega,\left(D_{V_{n}}^{\omega}, \mathscr{F}_{n}^{\omega}\right)$ generates a Markov process $X^{(n), \omega}=\left(X_{t}^{(n), \omega}\right)_{t \geqslant 0}$ such that $X_{t}^{(n), \omega}=n^{-1} X_{n^{\alpha} t}^{V_{1}, \omega}$ for all $t \geqslant 0$. Here and what follows, "=" means two processes enjoy the same distribution.

Now, consider the Dirichlet form $\left(D_{0}, \mathscr{F}_{0}\right)$ given by (4.6), i.e.,

$$
\begin{aligned}
D_{0}(f, f) & =\frac{1}{2} \int_{\{F \times F \backslash \operatorname{diag}\}}(f(x)-f(y))^{2} \frac{c(x, y)}{\rho(x, y)^{d+\alpha}} m(d x) m(d y), \quad f \in \mathscr{F}_{0}, \\
\mathscr{F}_{0} & =\left\{f \in L^{2}(F ; m): D_{0}(f, f)<\infty\right\},
\end{aligned}
$$

where $\alpha \in(0,2)$, diag $:=\{(x, y) \in F \times F: x=y\}$, and $c: F \times F \rightarrow(0, \infty)$ is a symmetric continuous function such that $0<c_{1} \leqslant c(x, y) \leqslant c_{2}<\infty$ for all $(x, y) \in F \times F \backslash$ diag and some constants $c_{1}, c_{2}$. We suppose that assumption (Dir.) holds. Let $Y:=\left(\left(Y_{t}\right)_{t \geqslant 0},\left(\mathbb{P}_{x}^{Y}\right)_{x \in F}\right)$ be a $\alpha$-stable-like process on $F$.

We next apply Theorem 4.5 to prove the quenched invariance principle for $\left(X_{t}^{\omega}\right)_{t \geqslant 0}$ under some assumptions on $w_{x, y}$. We first assume that the following holds.

\section{Assumption (Den.)}

(i) $\mathbb{E}\left[w_{x, y}\right]=J_{1}(x, y)$ and $\mathbb{E}\left[w_{x, y}^{-1} \mathbb{1}_{\left\{w_{x, y}>0\right\}}\right]=J_{2}(x, y)$ for any $x, y \in V_{1}$, where $0<C_{1}<$ $J_{i}(x, y)<C_{2}<\infty$ for all $i=1,2$ and $x, y \in V_{1}$.

(ii) For every compact set $S \subseteq F$,

$$
\lim _{n \rightarrow \infty}\left[\sup _{x, y \in S \cap V_{n}}\left|J_{1}(n x, n y)-c(x, y)\right|\right]=0 .
$$

(iii) There exists a countable subset $\Xi \subset \operatorname{Lip}_{c}(F)$ such that $\Xi$ is dense in $\operatorname{Lip}_{c}(F)$ under the uniform topology, i.e., for every $f \in \operatorname{Lip}_{c}(F)$,

$$
\inf _{g \in \Xi} \sup _{x \in F}|g(x)-f(x)|=0 .
$$

Remark 5.2. Obviously when $F=\mathbb{R}^{d}$ and $m$ is the Lebesgue measure, it follows from (5.2) that for any $x \neq y \in \mathbb{R}^{d}$ and $s \neq 0, c(x, y)=c(s x, s y)$, which implies that the limit process $\left(Y_{t}\right)_{t \geqslant 0}$ satisfies the scaling invariant property as follows

$$
\mathbb{P}_{\varepsilon^{-1} x}^{Y}\left(\left(\varepsilon Y_{t \varepsilon^{-\alpha}}\right)_{t \geqslant 0} \in A\right)=\mathbb{P}_{x}^{Y}\left(\left(Y_{t}\right)_{t \geqslant 0} \in A\right)
$$

for any $x \in \mathbb{R}^{d}, \varepsilon>0$ and $A \subset \mathscr{D}\left([0, \infty) ; \mathbb{R}^{d}\right)$.

For $\varepsilon>0, x \in V_{1}, R, r>0, c_{0}>1 / 2, c_{0}^{*} \geqslant 2$ and a bounded function $h$ on $V_{1} \times V_{1}$, define

$$
\begin{aligned}
p_{1}(r, R, \varepsilon) & =\mathbb{P}\left(\left|\sum_{x, y \in V_{1}: \rho(0, x) \leqslant R, \rho(x, y) \leqslant r}\left(w_{x, y}-J_{1}(x, y)\right)\right|>\varepsilon r^{d} R^{d}\right), \\
p_{2}(x, r, \varepsilon) & =\mathbb{P}\left(\left|\sum_{y \in V_{1}: \rho(x, y) \leqslant r}\left(w_{x, y}-J_{1}(x, y)\right)\right|>\varepsilon r^{d}\right), \\
p_{3}(x, r, \varepsilon) & =\mathbb{P}\left(\left|\sum_{y \in V_{1}: \rho(x, y) \leqslant r} \frac{\left(w_{x, y}-J_{1}(x, y)\right)}{\rho(x, y)^{d+\alpha-2}}\right|>\varepsilon r^{2-\alpha}\right), \\
p_{3}^{*}(x, r, \varepsilon) & =\mathbb{P}\left(\left|\sum_{y \in V_{1}: \rho(x, y) \leqslant r} \frac{\left(w_{x, y}-J_{1}(x, y)\right)}{\rho(x, y)^{d+\alpha-1}}\right|>\varepsilon r^{1-\alpha}\right), \quad \alpha \in(0,1), \\
p_{4}\left(x, r, c_{0}^{*}, \varepsilon\right) & =\mathbb{P}\left(\left|\sum_{y \in V_{1}: \rho(x, y) \leqslant c_{0}^{*} r}\left(w_{x, y}^{-1}-J_{2}(x, y)\right)\right|>\varepsilon r^{d}\right), \\
p_{5}^{(n)}(x, R, r, h, \varepsilon) & =\mathbb{P}\left(\left|\sum_{y \in B_{F}(0, n R) \cap V_{1}:} h(x, y) \frac{\left(w_{x, y}-J_{1}(x, y)\right)}{\rho(x, y)^{d+\alpha}}\right|^{2}>\varepsilon(n r)^{-2 \alpha}\right), \\
p_{6}\left(x, z, y, r, c_{0}\right) & =\mathbb{P}\left(\frac{\mu_{1}\left\{y \in V_{1}: \rho(y, x) \leqslant r, w_{y, z}>0\right\}}{\mu_{1}\left\{y \in V_{1}: \rho(y, x) \leqslant r\right\}} \leqslant c_{0}\right) .
\end{aligned}
$$


Theorem 5.3. Suppose that assumption (Den.) holds, and that there exists a constant $\theta \in(0,1)$ such that

(i) for any $\varepsilon_{0}$ and $\varepsilon$ small enough, any $N$ large enough, and any sequence of bounded function $\left\{h_{n}\right\}_{n \geqslant 1}$ on $V_{1} \times V_{1}$ with $\sup _{n \geqslant 1}\left\|h_{n}\right\|_{\infty}<\infty$,

$$
\begin{gathered}
\sum_{R=1}^{\infty} \sum_{r=1}^{R} p_{1}\left(r, R, \varepsilon_{0}\right)<\infty, \\
\sum_{R=1}^{\infty} \sum_{x \in B_{F}(0,6 R) \cap V_{1}} \sum_{r=R^{\theta} / 2}^{\infty} p_{2}\left(x, r, \varepsilon_{0}\right)<\infty,
\end{gathered}
$$

and$$
\sum_{n=1}^{\infty} \sum_{x \in B_{F}(0, n N) \cap V_{1}} p_{5}^{(n)}\left(x, N, \varepsilon, h_{n}, \varepsilon_{0}\right)<\infty .
$$

(ii) any $\varepsilon_{0}$ small enough,

$$
\sum_{R=1}^{\infty} \sum_{x \in B_{F}(0,6 R) \cap V_{1}} p_{3}\left(x, R^{\theta}, \varepsilon_{0}\right)<\infty
$$

and

$$
\sum_{R=1}^{\infty} \sum_{x \in B_{F}(0,6 R) \cap V_{1}} \sum_{r=R^{\theta} / 2}^{2 R} p_{4}\left(x, r, c_{0}^{*}, \varepsilon_{0}\right)<\infty,
$$

for any fixed $c_{0}^{*} \geqslant 0$, as well as

$$
\sum_{R=1}^{\infty} \sum_{x, z \in B_{F}(0,6 R) \cap V_{1}} \sum_{r=R^{\theta} / 2}^{2 R} p_{6}\left(x, z, r, c_{0}\right)<\infty
$$

for some fixed $c_{0}>1 / 2$.

When $\alpha \in(0,1),(5.7)$ can be replaced by

$$
\sum_{R=1}^{\infty} \sum_{x \in B_{F}(0,6 R) \cap V_{1}} p_{3}^{*}\left(x, R^{\theta}, \varepsilon_{0}\right)<\infty .
$$

Then for $\mathbb{P}$-a.s. $\omega \in \Omega$ and any $\left\{x_{n} \in V_{n}: n \geqslant 1\right\}$ such that $\lim _{n \rightarrow \infty} x_{n}=x$ with some $x \in F$, it holds that for every $T>0, \mathbb{P}_{x_{n}}^{(n), \omega}$ converges weakly to $\mathbb{P}_{x}^{Y}$ on the space of all probability measures on $\mathscr{D}([0, T] ; F)$, where $\mathbb{P}_{x_{n}}^{(n), \omega}$ denotes the distribution of process $X_{t}^{(n), \omega}=n^{-1} X_{n^{\alpha} t}^{V_{1}, \omega}$.

Theorem 5.3 immediately holds by applying Theorem 4.5, Lemmas 5.4 and 5.5 below to process $X_{t}^{(n), \omega}$.

Lemma 5.4. Under assumption (i) in Theorem 5.3, for $\mathbb{P}$-a.s. $\omega \in \Omega$, Assumption (Mos.) holds for the conductance $\left\{w_{x, y}^{(n)}(\omega)\right\}$.

Proof. Under (5.4), for any $\varepsilon_{0}>0$,

$$
\begin{aligned}
& \sum_{R=1}^{\infty} \mathbb{P}\left(\bigcup_{r=1}^{R}\left\{\left|\sum_{x, y \in V_{1}: \rho(0, x) \leqslant R, \rho(x, y) \leqslant r}\left(w_{x, y}-J_{1}(x, y)\right)\right|>\varepsilon_{0} r^{d} R^{d}\right\}\right) \\
& \leqslant \sum_{R=1}^{\infty} \sum_{r=1}^{R} \mathbb{P}\left(\left|\sum_{x, y \in V_{1}: \rho(0, x) \leqslant R, \rho(x, y) \leqslant r}\left(w_{x, y}-J_{1}(x, y)\right)\right|>\varepsilon_{0} r^{d} R^{d}\right)=\sum_{R=1}^{\infty} \sum_{r=1}^{R} p_{1}\left(r, R, \varepsilon_{0}\right)<\infty .
\end{aligned}
$$


Since $C_{1} \leqslant J_{1}(x, y) \leqslant C_{2}$ for all $x, y \in V_{1}$ and some positive constants $C_{1}$ and $C_{2}$, by the BorelCantelli lemma, we know that, for $\mathbb{P}$-a.s. $\omega \in \Omega$, there exists a constant $R_{0}(\omega) \geqslant 1$ such that for every $R>R_{0}(\omega)$,

$$
c_{1} r^{d} R^{d} \leqslant \sum_{x, y \in V_{1}: \rho(0, x) \leqslant R, \rho(x, y) \leqslant r} w_{x, y}(\omega) \leqslant c_{2} r^{d} R^{d}, \quad \forall 1 \leqslant r \leqslant R,
$$

where $c_{1}, c_{2}$ are positive constants independent of $\omega$. Then, for any $0<2 \eta<N$ and $n N>R_{0}(\omega)$, we have

$$
\begin{aligned}
& n^{-2 d} \sum_{x, y \in B_{F}(0, N) \cap V_{n}: 0<\rho(x, y) \leqslant \eta} \frac{w_{n x, n y}(\omega)}{\rho(x, y)^{d+\alpha-2}} \\
& \leqslant n^{-d+\alpha-2} \sum_{k=0}^{[\log (n \eta) / \log 2]+1} \sum_{x, y \in V_{1}: \rho(0, x) \leqslant n N \text { and } 2^{k} \leqslant \rho(x, y)<2^{k+1}} \frac{w_{x, y}(\omega)}{\rho(x, y)^{d+\alpha-2}} \\
& \leqslant n^{-d+\alpha-2} \sum_{k=0}^{[\log (n \eta) / \log 2]+1} \sum_{x, y \in V_{1}: \rho(0, x) \leqslant n N \text { and } 2^{k} \leqslant \rho(x, y)<2^{k+1}} w_{x, y}(\omega) \\
& \leqslant c_{3} n^{-d+\alpha-2} \sum_{k=0}^{[\log (n \eta) / \log 2]+1} 2^{-k(d+\alpha-2)} 2^{(k+1) d}(n N)^{d} \leqslant c_{4} N^{d} \eta^{2-\alpha} .
\end{aligned}
$$

This yields that (4.8) holds for $\mathbb{P}$-a.s. $\omega \in \Omega$.

According to (5.5), for every $\varepsilon_{0}>0$ small enough,

$$
\begin{aligned}
& \sum_{R=1}^{\infty} \mathbb{P}\left(\bigcup_{x \in B_{F}(0,6 R) \cap V_{1}} \bigcup_{r=R^{\theta} / 2}^{\infty}\left\{\left|\sum_{y \in V_{1}: \rho(x, y) \leqslant r}\left(w_{x, y}-J_{1}(x, y)\right)\right|>\varepsilon_{0} r^{d}\right\}\right) \\
& \leqslant \sum_{R=1}^{\infty} \sum_{x \in B_{F}(0,6 R) \cap V_{1}} \sum_{r=R^{\theta} / 2}^{\infty} \mathbb{P}\left(\left\{\left|\sum_{y \in V_{1}: \rho(x, y) \leqslant r}\left(w_{x, y}-J_{1}(x, y)\right)\right|>\varepsilon_{0} r^{d}\right\}\right) \\
& \leqslant \sum_{R=1}^{\infty} \sum_{x \in B_{F}(0,6 R)} \sum_{r=R^{\theta} / 2}^{\infty} p_{2}\left(x, r, \varepsilon_{0}\right)<\infty .
\end{aligned}
$$

Hence, by the Borel-Cantelli lemma, we can find a constant $R_{1}(\omega)>0$ such that for every $R>R_{1}(\omega)$, $x \in B_{F}(0,6 R) \cap V_{1}$ and $r \geqslant R^{\theta} / 2,\left|\sum_{y \in V_{1}: \rho(x, y) \leqslant r}\left(w_{x, y}-J_{1}(x, y)\right)\right| \leqslant \varepsilon_{0} r^{d}$. Due to the fact that $0<C_{1} \leqslant J_{1}(x, y) \leqslant C_{2}<\infty$ for any $x, y \in V_{1}$ again, we arrive at the statement that for all $R>R_{1}(\omega)$,

$$
c_{5} r^{d} \leqslant \sum_{y \in V_{1}: \rho(x, y) \leqslant r} w_{x, y} \leqslant c_{6} r^{d}, \quad \forall x \in B_{F}(0,6 R), r \geqslant R^{\theta} / 2 .
$$

Therefore, by (5.11), for every $n, j \geqslant 1$ large enough such that $2 n N>R_{1}(\omega)$ and $j>N$,

$$
\begin{aligned}
& n^{-2 d} \sum_{x, y \in B_{F}(0, N) \cap V_{n}: \rho(x, y) \geqslant j} \frac{w_{n x, n y}(\omega)}{\rho(x, y)^{d+\alpha}} \\
& \leqslant n^{-d+\alpha} \sum_{x \in V_{1}: \rho(0, x) \leqslant n N} \sum_{y \in V_{1}: \rho(x, y) \geqslant n j} \frac{w_{x, y}(\omega)}{\rho(x, y)^{d+\alpha}} \\
& \leqslant n^{-d+\alpha} \sum_{x \in V_{1}: \rho(0, x) \leqslant n N} \sum_{k=\left[\frac{\log (n j)}{\log 2}\right]} 2^{-k(d+\alpha)} \sum_{y \in V_{1}: \rho(x, y) \leqslant 2^{k+1}} w_{x, y}(\omega) \\
& \leqslant c_{7} n^{-d+\alpha} \sum_{x \in V_{1}: \rho(0, x) \leqslant n N} \sum_{k=\left[\frac{\log (n j)}{\log 2}\right]}^{\infty} 2^{-k(d+\alpha)} 2^{(k+1) d} \leqslant c_{8} N^{d} j^{-\alpha} .
\end{aligned}
$$

Hence, letting $n \rightarrow \infty$ first and then $j \rightarrow \infty$, we prove that (4.9) holds for P-a.s. $\omega \in \Omega$. 
Given $f \in \operatorname{Lip}_{c}(F)$, let

$$
h_{n}(x, y):= \begin{cases}f\left(n^{-1} y\right)-f\left(n^{-1} x\right), & n^{-1} x, n^{-1} y \in V_{n}, \\ 0, & \text { otherwise. }\end{cases}
$$

Let $\mathbb{Q}_{+}$be the set of all positive rational numbers. Applying (5.6) to $h_{n}(x, y)$ and using the BorelCantelli lemma, we can find a null set $\Phi(f)$ so that for every $\omega \notin \Phi(f), \varepsilon, \varepsilon_{0} \in \mathbb{Q}_{+}$small enough and $R \in \mathbb{Q}_{+}$large enough, there exists a constant $n_{0}(\omega)>0$ (which may depend on $\varepsilon_{0}, \varepsilon, N$ and $f$ ) such that for every $n>n_{0}(\omega)$ and $x \in B_{F}(0, n R) \cap V_{1}$,

$$
\left|\sum_{y \in B_{F}(0, n R) \cap V_{1}: \rho(x, y) \geqslant n \varepsilon}\left(f\left(n^{-1} y\right)-f\left(n^{-1} x\right)\right) \frac{\left(w_{x, y}(\omega)-J_{1}(x, y)\right)}{\rho(x, y)^{d+\alpha}}\right|^{2} \leqslant \varepsilon_{0}(n \varepsilon)^{-2 \alpha} .
$$

Then, for $n$ large enough such that $n \varepsilon>(n R)^{\theta}$, we have

$$
\begin{aligned}
& n^{-d} \sum_{x \in B_{F}(0, R) \cap V_{n}}\left(\sum_{\substack{y \in B_{F}(0, R) \cap V_{n}: \\
\rho(x, y)>\varepsilon}}(f(x)-f(y)) \frac{\left(w_{n x, n y}(\omega)-J_{1}(n x, n y)\right)}{\rho(x, y)^{d+\alpha}} m_{n}(y)\right)^{2} \\
& =n^{-d+2 \alpha} \sum_{x \in B_{F}(0, n R) \cap V_{1}}\left(\sum_{y \in B_{F}(0, n R) \cap V_{1}: \rho(x, y)>n \varepsilon} h_{n}(x, y) \frac{\left(w_{x, y}(\omega)-J_{1}(x, y)\right)}{\rho(x, y)^{d+\alpha}}\right)^{2} \\
& \leqslant n^{-d+2 \alpha} \sum_{x \in B_{F}(0, n R) \cap V_{1}} \varepsilon_{0}(n \varepsilon)^{-2 \alpha} \leqslant c_{9} R^{d} \varepsilon^{-2 \alpha} \varepsilon_{0} .
\end{aligned}
$$

On the other hand, due to (5.2), we can verify that every fixed $R>0$ and $\varepsilon>0$,

$$
\begin{aligned}
& \lim _{n \rightarrow \infty} n^{-d} \sum_{x \in B_{F}(0, R) \cap V_{n}}\left(\sum_{\substack{y \in B_{F}(0, R) \cap V_{n}: \\
\rho(x, y)>\varepsilon}}(f(x)-f(y)) \frac{\left(J_{1}(n x, n y)-c(x, y)\right)}{\rho(x, y)^{d+\alpha}} m_{n}(y)\right)^{2} \\
& \leqslant 4\|f\|_{\infty}^{2} \varepsilon^{-2(d+\alpha)} \lim _{n \rightarrow \infty} n^{-3 d} \sum_{x \in B_{F}(0, R) \cap V_{n}}\left(\sum_{y \in B_{F}(0, R) \cap V_{n}: \rho(x, y)>\varepsilon}\left|J_{1}(n x, n y)-c(x, y)\right|\right)^{2} \\
& \leqslant c_{10}\|f\|_{\infty}^{2} \varepsilon^{-2(d+\alpha)} R^{d} \lim _{n \rightarrow \infty}\left\{n^{-2 d} \sum_{x, y \in B_{F}(0, R) \cap V_{n}}\left(J_{1}(n x, n y)-c(x, y)\right)^{2}\right\}=0 .
\end{aligned}
$$

Combining two estimates above, we can obtain that for every $f \in \operatorname{Lip}_{c}(F)$, (4.10) holds for all $\omega \notin \Phi(f)$ and $\varepsilon, R \in \mathbb{Q}_{+}$with $\varepsilon$ small enough and $R$ large enough, by first letting $n \rightarrow \infty$ and then taking $\varepsilon_{0} \rightarrow 0$.

For the countable subset $\Xi \subset \operatorname{Lip}_{c}(F)$ in Assumption (Den.)(iii), we define $\Phi_{1}:=\cup_{f \in \Xi} \Phi(f)$. Obviously $\Phi_{1}$ is a null set. It is also easy to see that for every $\omega \notin \Phi_{1}, f \in \Xi$ and $\varepsilon, R \in \mathbb{Q}_{+}$with $\varepsilon$ small enough and $R$ large enough,

$$
\lim _{n \rightarrow \infty} T_{n}(f, \varepsilon, R, \omega)=0
$$

where

$$
T_{n}(f, \varepsilon, R, \omega):=n^{-d} \sum_{x \in B_{F}(0, R) \cap V_{n}}\left(\sum_{y \in B_{F}(0, R) \cap V_{n}: \rho(x, y)>\varepsilon}(f(y)-f(x)) \frac{w_{n x, n y}(\omega)-c(x, y)}{\rho(x, y)^{d+\alpha}} m_{n}(y)\right)^{2} .
$$

Furthermore, note that for every $f, g \in \operatorname{Lip}_{c}(F)$ and $\varepsilon, R>0$,

$$
\begin{aligned}
& T_{n}(f-g, \varepsilon, R, \omega) \\
& \leqslant 4 n^{-d} \sup _{x \in F}|f(x)-g(x)|^{2} \cdot\left(\sum_{x \in B_{F}(0, R) \cap V_{n}}\left(\sum_{y \in B_{F}(0, R) \cap V_{n}: \rho(x, y) \geqslant \varepsilon} \frac{w_{n x, n y}(\omega)+c(x, y)}{\rho(x, y)^{d+\alpha}} m_{n}(y)\right)^{2}\right) \\
& \leqslant c_{11} n^{-3 d} \varepsilon^{-2(d+\alpha)} \sup _{x \in F}|f(x)-g(x)|^{2} \cdot\left[\sum_{x \in B_{F}(0, R) \cap V_{n}}\left(\sum_{y \in B_{F}(0, R) \cap V_{n}} w_{n x, n y}+c(x, y)\right)^{2}\right] .
\end{aligned}
$$


Then, by (5.11), we can find a null set $\Phi_{2} \in \Omega$ such that for each $\omega \notin \Phi_{2}$, there exists $n_{0}(\omega) \geqslant 1$ so that for all $n>n_{0}(\omega), f, g \in \operatorname{Lip}_{c}(F)$ and $\varepsilon, R>0$,

$$
T_{n}(f-g, \varepsilon, R, \omega) \leqslant c_{12} \varepsilon^{-2(d+\alpha)} R^{3 d} \sup _{x \in F}|f(x)-g(x)|^{2} .
$$

Combining the estimate above with (5.3) yields that for each $f \in \operatorname{Lip}_{c}(F), \varepsilon, R>0$ and $\omega \notin \Phi_{2}$,

$$
\inf _{g \in \Xi} \limsup _{n \rightarrow \infty} T_{n}(f-g, \varepsilon, R, \omega)=0 .
$$

Let $\Phi:=\Phi_{1} \cup \Phi_{2}$. Then, $\Phi$ is a null set. According to (5.12), we know that for every $f \in \operatorname{Lip}_{c}(F)$, $g \in \Xi, \omega \notin \Phi$ and $\varepsilon, R \in \mathbb{Q}_{+}$with $\varepsilon$ small enough and $R$ large enough,

$$
\begin{aligned}
\limsup _{n \rightarrow \infty} T_{n}(f, \varepsilon, R, \omega) & \leqslant 2 \limsup _{n \rightarrow \infty} T_{n}(g, \varepsilon, R, \omega)+2 \limsup _{n \rightarrow \infty} T_{n}(f-g, \varepsilon, R, \omega) \\
& =2 \limsup _{n \rightarrow \infty} T_{n}(f-g, \varepsilon, R, \omega) .
\end{aligned}
$$

Therefore, taking infimum over all $g \in \Xi$ in the inequality above and applying (5.13), we can prove that (4.10) holds for every $f \in \operatorname{Lip}_{c}(F), \omega \notin \Phi$ and $\varepsilon, R \in \mathbb{Q}_{+}$with $\varepsilon$ small enough and $R$ large enough. By the same (and even simpler) approximation arguments as above, we can further verify that (4.10) holds for every $f \in \operatorname{Lip}_{c}(F), \omega \notin \Phi, \varepsilon>0$ small enough and $R>0$ large enough.

Since (4.11) can be proved in the similar way, we omit it here.

We note that the last part of the proof above that handles the null set carefully is motivated by the proof of [19, Theorem 1.1].

Lemma 5.5. Suppose that there exists a constant $\theta \in(0,1)$ such that condition (5.5) and assumption (ii) in Theorem 5.3 hold. Then for $\mathbb{P}$-a.s. $\omega \in \Omega$, Assumption (Wea. $(\theta)$ ) holds for the conductance $\left\{w_{x, y}^{(n)}(\omega)\right\}$.

Proof. First, according to (5.9), the property $\mu_{n}(A)=\mu_{1}(n A)$ and the definitions of $m_{n}$ and $w_{x, y}^{(n)}$, we can easily deduce from the Borel-Cantelli lemma that there is a constant $R_{0}(\omega)>0$ such that for any $R>R_{0}(\omega)$ and $R^{\theta} / 2 \leqslant r \leqslant R,(4.15)$ holds.

By (5.7),

$$
\begin{aligned}
& \sum_{R=1}^{\infty} \mathbb{P}\left(\bigcup_{x \in B_{F}(0,6 R) \cap V_{1}}\left\{\left|\sum_{y \in V_{1}: \rho(x, y) \leqslant R^{\theta}} \frac{\left(w_{x, y}-J_{1}(x, y)\right)}{\rho(x, y)^{d+\alpha-2}}\right|>\varepsilon_{0} R^{\theta(2-\alpha)}\right\}\right) \\
& \leqslant \sum_{R=1}^{\infty} \sum_{x \in B_{F}(0,6 R) \cap V_{1}} \mathbb{P}\left(\left|\sum_{y \in V_{1}: \rho(x, y) \leqslant R^{\theta}} \frac{\left(w_{x, y}-J_{1}(x, y)\right)}{\rho(x, y)^{d+\alpha-2}}\right|>\varepsilon_{0} R^{\theta(2-\alpha)}\right) \\
& =\sum_{R=1}^{\infty} \sum_{x \in B_{F}(0,6 R) \cap V_{1}} p_{3}\left(x, R^{\theta}, \varepsilon_{0}\right)<\infty .
\end{aligned}
$$

Hence, by the Borel-Cantelli lemma, there exists a constant $R_{0}(\omega)>0$ such that for any $R>R_{0}(\omega)$,

$$
\sum_{y \in V_{1}: \rho(x, y) \leqslant R^{\theta}} \frac{w_{x, y}}{\rho(x, y)^{d+\alpha-2}} \leqslant c_{1} R^{\theta(2-\alpha)}, \quad \forall x \in B_{F}(0,6 R) \cap V_{1} .
$$

Furthermore, using (5.11) and choosing $\varepsilon_{0}$ small enough and $R_{0}(\omega)$ large enough, we find that for every $R>R_{0}(\omega)$,

$$
c_{2}^{-1} r^{d} \leqslant \sum_{y \in V_{1}: \rho(x, y) \leqslant r} w_{x, y} \leqslant c_{2} r^{d}, \quad \forall r>R^{\theta} / 2, x \in B_{F}(0,6 R) \cap V_{1} .
$$

Combining this with (5.14), we see that for every $R>R_{0}(w), x \in B_{F}(0,6 R / n) \cap V_{n}$, and $R^{\theta} / 2 \leqslant$ $r \leqslant 2 R$,

$$
n^{-(d+\alpha-2)} \sum_{y \in V_{n}: \rho(x, y) \leqslant r / n} \frac{w_{x, y}^{(n)}}{\rho(x, y)^{d+\alpha-2}}
$$




$$
\begin{aligned}
& \leqslant \sum_{y \in V_{1}: \rho(x, y)<R^{\theta} / 2} \frac{w_{x, y}}{\rho(x, y)^{d+\alpha-2}}+\sum_{k=\left[\log \left(R^{\theta} / 2\right) / \log 2\right]}^{[\log r / \log 2]+1} 2^{-k(d+\alpha-2)}\left(\sum_{y \in V_{1}: 2^{k}<\rho(x, y) \leqslant 2^{k+1}} w_{x, y}\right) \\
& \leqslant c_{4}\left(R^{\theta(2-\alpha)}+\sum_{k=\left[\log \left(R^{\theta} / 2\right) / \log 2\right]}^{[\log r / \log 2]+1} 2^{-k(\alpha-2)}\right) \leqslant c_{5} r^{2-\alpha} .
\end{aligned}
$$

Therefore, (4.14) holds for $\mathbb{P}$-a.s. $\omega \in \Omega$.

Due to (5.15) again, we know that for every $R>R_{0}(\omega), x \in B_{F}(0,6 R / n) \cap V_{n}$ and $r>R^{\theta} / 2$,

$$
\begin{aligned}
n^{-(d+\alpha)} \sum_{y \in V_{n}: \rho(x, y)>r / n} \frac{w_{x, y}^{(n)}}{\rho_{n}(x, y)^{d+\alpha}} & \leqslant \sum_{k=[\log r / \log 2]}^{\infty} 2^{-k(d+\alpha)}\left(\sum_{y \in V_{1}: 2^{k}<\rho(x, y) \leqslant 2^{k+1}} w_{x, y}\right) \\
& \leqslant c_{6} \sum_{k=[\log r / \log 2]}^{\infty} 2^{-k(d+\alpha)} 2^{d(k+1)} \leqslant c_{7} r^{-\alpha},
\end{aligned}
$$

which implies that (4.18) is satisfied for P-a.s. $\omega \in \Omega$.

Following the arguments above, and using (5.8) and the Borel-Cantelli lemma, we can obtain that (4.16) holds for P-a.s. $\omega \in \Omega$. On the other hand, when $\alpha \in(0,1)$, we can use (5.10) to prove that (4.17) holds for P-a.s. $\omega \in \Omega$. The proof is complete.

5.2. Examples. As an application of Theorem 5.3, we consider four examples. One is a lattice on a half/quarter space, and other three are time-change of stable-like processes, a bounded Lipschitz domain and a fractal graph respectively. We also show the the quenched invariance principle for a class of constant speed $\alpha$-stable-like random walks on $\mathbb{Z}^{d}$, by using Theorem 1.1 and the time change argument.

5.2.1. Lattice on a half/quarter space. Let $F:=\mathbb{R}_{+}^{d_{1}} \times \mathbb{R}^{d_{2}}$ with $d_{1}, d_{2} \in \mathbb{N} \cup\{0\}$, and $\rho$ and $m$ be the Euclidean distance and the Lebesgue measure respectively, which clearly satisfy assumption (MMS). Therefore the process $Y$ associated with Dirichlet form $\left(D_{0}, \mathscr{F}_{0}\right)$ is a reflected stable-like process on $F$, see e.g. [24]. Obviously $\left(D_{0}, \mathscr{F}_{0}\right)$ satisfies assumption (Dir.). Here we will take $V_{1}=\mathbb{L}:=\mathbb{Z}_{+}^{d_{1}} \times \mathbb{Z}^{d_{2}}$, and $K_{n} \equiv 1$ for all $n \in \mathbb{N}$. Note that the scaling limit of $n^{-1} \mathbb{L}$ is $F$.

Let $E:=\{(x, y): x, y \in \mathbb{L}\}$ be the collection of unordered pairs on $\mathbb{L},\left\{w_{x, y}:(x, y) \in E\right\}$ be a sequence of non-negative independent random variables, and $\left(X_{t}^{\omega}\right)_{t \geqslant 0}$ be the Markov process with infinitesimal generator $L_{\mathbb{L}}^{\omega}$ defined by (1.1). Obviously $\left(X_{t}^{\omega}\right)_{t \geqslant 0}$ is the symmetric Hunt process associated with the Dirichlet form $\left(D_{V_{1}}^{\omega}, \mathscr{F}_{1}^{\omega}\right)$ with $V_{1}=\mathbb{L}$ and $w_{x, y}^{(1)}(\omega)=w_{x, y}(\omega)$.

Proposition 5.6. Let $d:=d_{1}+d_{2}>4-2 \alpha$. Suppose that $\left\{w_{x, y}:(x, y) \in E\right\}$ is a sequence of non-negative independent random variables satisfying that

$$
\sup _{x, y \in \mathbb{L}, x \neq y} \mathbb{P}\left(w_{x, y}=0\right)<1 / 2
$$

and

$$
\sup _{x, y \in \mathbb{L}} \mathbb{E}\left[w_{x, y}^{2 p}\right]<\infty \text { and } \sup _{x, y \in \mathbb{L}} \mathbb{E}\left[w_{x, y}^{-2 q} \mathbb{1}_{\left\{w_{x, y}>0\right\}}\right]<\infty
$$

with $p>\max \{(d+2) / d,(d+1) /(2(2-\alpha))\}$ and $q>(d+2) / d$. If moreover (5.2) holds true, then the quenched invariance principle holds for $X^{\omega}$. with the limit process $Y$. Moreover, when $\alpha \in(0,1)$, the conclusion still holds true for $d>2-2 \alpha$, if $p>\max \{(d+1) /(2(1-\alpha)),(d+2) / d\}$ and $q>(d+2) / d$.

Proof. According to Theorem 5.3, it suffices to verify (5.4) - (5.10). We first verify (5.9). Suppose that $p_{0}:=\sup _{x, y \in \mathbb{L}, x \neq y} \mathbb{P}\left(w_{x, y}=0\right)<1 / 2$. Denote by $L(x, r):=|\{y \in \mathbb{L}:|y-x| \leqslant r\}|$. Let $\left\{\eta_{y}\right\}_{\{y \in \mathbb{L}:|y-x| \leqslant r\}}$ be a sequence of i.i.d. Bernoulli random variables (possibly on the extended probability space) such that $\mathbb{P}\left(\eta_{y}=1\right)=1-p_{0}, \mathbb{P}\left(\eta_{y}=0\right)=p_{0}$ and $\eta_{y} \leqslant \mathbb{1}_{\left\{w_{z, y} \neq 0\right\}}$ a.s.. Take $1 / 2<c_{0}<1-p_{0}$, then for every $r>0$ and $x, z \in \mathbb{L}$,

$$
\mathbb{P}\left(\sum_{y \in \mathbb{L}:|y-x| \leqslant r} \mathbb{1}_{\left\{w_{z, y} \neq 0\right\}} \leqslant c_{0} L(x, r)\right) \leqslant \mathbb{P}\left(\sum_{y \in \mathbb{L}:|y-x| \leqslant r} \eta_{y} \leqslant c_{0} L(x, r)\right)
$$




$$
\begin{aligned}
& =\mathbb{P}\left(\frac{\sum_{y \in \mathbb{L}:|y-x| \leqslant r}\left(\eta_{y}-\mathbb{E}\left[\eta_{y}\right]\right)}{L(x, r)} \leqslant c_{0}-\left(1-p_{0}\right)\right) \\
& \leqslant c^{\prime} e^{-c^{\prime \prime} r^{d}},
\end{aligned}
$$

where in the first inequality we used the fact that $\sum_{y \in \mathbb{L}:|y-x| \leqslant r} \eta_{y} \leqslant \sum_{y \in \mathbb{L}:|y-x| \leqslant r} \mathbb{1}_{\left\{w_{z, y} \neq 0\right\}}$ a.s., and the last inequality follows from the Cramer theorem for i.i.d. Bernoulli random variables and the fact $L(x, r) \asymp r^{d}$. The estimate above yields that

$$
\sum_{R=1}^{\infty} \sum_{x, z \in B_{F}(0,6 R) \cap V_{1}} \sum_{r=R^{\theta} / 2}^{2 R} p_{6}\left(x, z, r, c_{0}\right)<\infty .
$$

This is, (5.9) holds with $c_{0}$ chosen above.

Recall that, for a sequence of mutually independent random variables $\left\{\eta_{n}\right\}_{n \geqslant 1}$ satisfying $\mathbb{E}\left[\eta_{n}\right]=0$ for every $n \in \mathbb{N}_{+}, M_{n}:=\sum_{k=1}^{n} \eta_{k}$ is a martingale with respect to the natural filtration. Then, by the Burkholder-Gundy-Davis inequality, for any $l \geqslant 2$ and $n \in \mathbb{N}_{+}$,

$$
\mathbb{E}\left[\left|\sum_{k=1}^{n} \eta_{k}\right|^{l}\right]=\mathbb{E}\left[\left|M_{n}\right|^{l}\right] \leqslant c_{1} \mathbb{E}\left[\langle M\rangle_{n}^{l / 2}\right] \leqslant c_{2} n^{l / 2-1} \sum_{k=1}^{n} \mathbb{E}\left[\left|\eta_{k}\right|^{l}\right],
$$

where $\langle M\rangle_{n}=\sum_{k=1}^{n} \eta_{k}^{2}$ is the variational process associated with $M_{n}$, and $c_{1}, c_{2}>0$ are independent of $n$.

Hence, by (5.17), we know that for every $\varepsilon_{0}>0, R, r>0, c_{0}^{*} \geqslant 2, n \geqslant 1$ and a subsequence of bounded measurable functions $\left\{h_{n}\right\}_{n \geqslant 1}$ on $\mathbb{L} \times \mathbb{L}$ such that $\sup _{n \geqslant 1}\left\|h_{n}\right\|_{\infty}<\infty$,

$$
\begin{aligned}
p_{1}\left(r, R, \varepsilon_{0}\right) \leqslant & \varepsilon_{0}^{-2 p} R^{-2 p d} r^{-2 p d} \mathbb{E}\left[\left|\sum_{x, y \in \mathbb{L}:|x| \leqslant R,|y-x| \leqslant r}\left(w_{x, y}-\mathbb{E}\left[w_{x, y}\right]\right)\right|^{2 p}\right] \leqslant c_{3}\left(\varepsilon_{0}\right) r^{-p d} R^{-p d}, \\
p_{2}\left(x, r, \varepsilon_{0}\right) \leqslant & \varepsilon_{0}^{-2 p} r^{-2 p d} \mathbb{E}\left[\left|\sum_{y \in \mathbb{L}:|y-x| \leqslant r}\left(w_{x, y}-\mathbb{E}\left[w_{x, y}\right]\right)\right|^{2 p}\right] \leqslant c_{4}\left(\varepsilon_{0}\right) r^{-p d}, \\
p_{4}\left(x, r, c_{0}^{*}, \varepsilon_{0}\right) \leqslant & \varepsilon_{0}^{-2 q} r^{-2 q d} \mathbb{E}\left[\left|\sum_{y \in \mathbb{L}:|y-x| \leqslant c_{0}^{*} r}\left(w_{x, y}^{-1}-\mathbb{E}\left[w_{x, y}^{-1}\right]\right)\right|^{2 q}\right] \leqslant c_{5}\left(\varepsilon_{0}, c_{0}^{*}\right) r^{-q d}, \\
p_{5}^{(n)}\left(x, N, \varepsilon, h_{n}, \varepsilon_{0}\right) \leqslant & c_{6}\left(\varepsilon_{0}\right) n^{2 \alpha p} \cdot(n \varepsilon)^{-2 p(d+\alpha)} \\
& \times \mathbb{E}\left[\left|\sum_{y \in \mathbb{L}:|y-x| \geqslant n \varepsilon,|y| \leqslant n N} h_{n}(x, y)(n \varepsilon)^{d+\alpha} \frac{\left(w_{x, y}-\mathbb{E}\left[w_{x, y}\right]\right)}{|x-y|^{d+\alpha}}\right|^{2 p}\right] \\
\leqslant & c_{7}\left(\varepsilon_{0}, N, \varepsilon, \sup _{n \geqslant 1}\left\|h_{n}\right\|_{\infty}\right) n^{2 \alpha p} n^{p d} n^{-2 p(d+\alpha)}=c_{7}\left(\varepsilon_{0}, N, \varepsilon, \sup _{n \geqslant 1}\left\|h_{n}\right\|_{\infty}\right) n^{-p d} .
\end{aligned}
$$

In the following, we fix $x \in \mathbb{L}$. Set $\xi(y):=\frac{\left(w_{x, y}-\mathbb{E}\left[w_{x, y}\right]\right)}{\left.|x-y|\right|^{d+\alpha-2}}$ for every $y \in \mathbb{L}$ with $y \neq x$. Clearly, $\{\xi(y)\}_{y \in \mathbb{L}: y \neq x}$ are mutually independent. By (5.16), $\mathbb{E}[\xi(y)]=0$ and $\mathbb{E}\left[|\xi(y)|^{2 p}\right] \leqslant c_{8}|x-y|^{-2 p(d+\alpha-2)}$. Choosing $0<\delta<\frac{d+2 \alpha-4}{d}$ (thanks to $d+2 \alpha-4>0$ ) and applying the first inequality in (5.17), we arrive at that for every $r \geqslant 1$

$$
\begin{aligned}
& \mathbb{E}\left[\left|\sum_{y \in \mathbb{L}:|y-x| \leqslant r} \frac{\left(w_{x, y}-\mathbb{E}\left[w_{x, y}\right]\right)}{|x-y|^{d+\alpha-2}}\right|^{2 p}\right] \\
& =\mathbb{E}\left[\left|\sum_{y \in \mathbb{L}:|y-x| \leqslant r} \xi(y)\right|^{2 p}\right] \leqslant c_{9} \mathbb{E}\left[\left.\left.\left|\sum_{y \in \mathbb{L}:|y-x| \leqslant r}\right| \xi(y)\right|^{2}\right|^{p}\right] \\
& \leqslant c_{9} \mathbb{E}\left[\left(\sum_{y \in \mathbb{L}:|y-x| \leqslant r}|\xi(y)|^{2 p}|x-y|^{d(p+\delta-1)}\right) \cdot\left(\sum_{y \in \mathbb{L}:|y-x| \leqslant r}|x-y|^{-\frac{d(p+\delta-1)}{p-1}}\right)\right] \leqslant c_{10} .
\end{aligned}
$$


Here the second inequality above follows from the Hölder inequality, in the last inequality we used $\mathbb{E}\left[|\xi(y)|^{2 p}\right] \leqslant c_{8}|x-y|^{-2 p(d+\alpha-2)}$, and $c_{9}, c_{10}$ are independent of $r$. Then, by the Markov inequality, we know that for every $x \in \mathbb{L}, p_{3}\left(x, R, \varepsilon_{0}\right) \leqslant c_{11}\left(\varepsilon_{0}\right) R^{-2(2-\alpha) p}$.

Under assumptions of the proposition, we can choose $\theta \in(0,1)$ (close to 1 ) such that

$$
p>\max \left\{\frac{d+1+\theta}{d \theta}, \frac{d+1}{2 \theta(2-\alpha)}\right\} \text { and } q>\frac{d+1+\theta}{d \theta},
$$

also thanks to the condition that $d>4-2 \alpha$ again. Then, according to all the estimates above, we know immediately that $(5.4)$ - (5.8) hold for this $\theta \in(0,1)$ and every sufficiently small $\varepsilon_{0}>0$.

Suppose that $\alpha \in(0,1)$. If $d>2-2 \alpha, p>\max \{(d+1) /(2(1-\alpha)),(d+2) / d\}$ and $q>(d+2) / d$, then we can choose $\theta \in(0,1)$ (close to 1 ) such that

$$
p>\max \left\{\frac{d+1+\theta}{d \theta}, \frac{d+1}{2 \theta(1-\alpha)}\right\} \text { and } q>\frac{d+1+\theta}{d \theta} .
$$

Following the argument above, we can prove that (5.4) - (5.6), (5.8) and (5.10) are satisfied. Then, the desired assertion follows from Theorem 5.3 again. The proof is complete.

Theorem 1.1 is a direct consequence of Proposition 5.6, since (5.2) holds trivially in this setting. We note that the idea of the proof above using the Burkholder-Gundy-Davis inequality comes from the proof of [19, Theorem 1.1].

5.2.2. Time-change of $\alpha$-stable-like process on $\mathbb{R}^{d}$. We can consider the case that the approximating measure $m_{n}$ is not $n^{-d} \mu_{n}$. Let us first fix the triple $(F, \rho, m)$ with $F=\mathbb{R}^{d}, \rho$ being the Euclidean distance and $m(d x)=K(x) d x$, where $d x$ denotes the Lebesgue measure on $\mathbb{R}^{d}$ and $K$ is a continuous function on $\mathbb{R}^{d}$ satisfying that $0<C_{1} \leqslant K(x) \leqslant C_{2}<\infty$ for some constants $C_{1} \leqslant C_{2}$. Then, the process $Y$ associated with the Dirichlet form $\left(D_{0}, \mathscr{F}_{0}\right)$ given at the beginning of Subsection 5.1 is a time-change of symmetric $\alpha$-stable process on $\mathbb{R}^{d}$ with $c(x, y)=K(x)^{-1} K(y)^{-1}$ for $x, y \in \mathbb{R}^{d}$. It is obvious that $\left(D_{0}, \mathscr{F}_{0}\right)$ satisfies assumption (Dir.).

Similar to the previous part, we can take $V_{1}=\mathbb{Z}^{d}$, and $m_{n}=n^{-d} K_{n} \mu_{n}$ with $\mu_{n}$ being the counting measure on $n^{-1} \mathbb{Z}^{d}$ and

$$
K_{n}(x)=n^{-d} \int_{U_{n}(x)} K(x) d x, \quad x \in n^{-1} \mathbb{Z}^{d},
$$

where $U_{n}(x)=\prod_{i=1}^{d}\left[x_{i}, x_{i}+n^{-1}\right)$ for any $x=\left(x_{1}, \cdots, x_{d}\right) \in n^{-1} \mathbb{Z}^{d}$.

Suppose that $E:=\{(x, y): x, y \in \mathbb{L}\}$ is the collection of unordered pairs on $\mathbb{L}$, and $\left\{w_{x, y}\right.$ : $(x, y) \in E\}$ is a sequence of non-negative independent random variables. Let $\left(X_{t}^{\omega, n}\right)_{t \geqslant 0}$ and $\left(Y_{t}^{\omega, n}\right)_{t \geqslant 0}$ be symmetric Hunt processes associated with the Dirichlet form $\left(D_{V_{n}}^{\omega}, \mathscr{F}_{n}^{\omega}\right)$ on $L^{2}\left(V_{n} ; m_{n}\right)$ and $L^{2}\left(V_{n} ; n^{-d} \mu_{n}\right)$ respectively, where

$$
\begin{aligned}
D_{V_{n}}^{\omega}(f, f) & =\sum_{x, y \in V_{n}}(f(x)-f(y))^{2} \frac{w_{n x, n y}}{|x-y|^{d+\alpha}}, \\
\mathscr{F}_{n}^{\omega} & =\left\{f \in L^{2}\left(V_{n} ; \mu_{n}\right): D_{V_{n}}^{\omega}(f, f)<\infty\right\} .
\end{aligned}
$$

Let $\left(X_{t}^{\omega}\right)_{t \geqslant 0}:=\left(X_{t}^{\omega, 1}\right)_{t \geqslant 0}$. It is easy to verify that $\frac{1}{n} X_{n^{\alpha} t}^{\omega}=Y_{A_{n, t}^{-1}}^{\omega, n}$ with $A_{n, t}:=\int_{0}^{t} K_{n}\left(Y_{s}^{\omega, n}\right) d s$ for any $t>0$.

Note that for any compact set $S \subset \mathbb{R}^{d}, \lim _{n \rightarrow \infty} \sup _{x \in S}\left|K_{n}\left([x]_{n}\right)-K(x)\right|=0$. Following the same arguments in the proof of Proposition 5.6, we can obtain that under assumption (5.16) the quenched invariance principle holds for $\left(X_{t}^{\omega}\right)_{t \geqslant 0}$ with limiting process $Y$ being a time-change of symmetric $\alpha$-stable process on $\mathbb{R}^{d}$.

Remark 5.7. From the example above, we know that to identity the limit process consists of two ingredients. One is to verify locally weak convergence of $m_{n}$ to $m$, and the other is to justify convergence of the jumping kernel for the associated Dirichlet form. In fact, by carefully tracking the proof above, we can see that if the measure $m_{n}$ is replaced by a more general (random) measure which converges locally weakly to $m$, then the quenched invariance principle still holds with the same limiting process. 
5.2.3. Bounded Lipschitz domain. In fact, Proposition 5.6 holds not only for a half/quarter space, but also for the closure of a bounded Lipschitz domain in $\mathbb{R}^{d}$, whose intrinsic distance is equivalent to the Euclidean distance and whose volume growth is with order $d$. In details, let $F \subset \mathbb{R}^{d}$ be a closed set such that for any $x, y \in F$ and $r>0, c_{1} r^{d} \leqslant m\left(B_{F}(x, r)\right) \leqslant c_{2} r^{d}$ and $c_{1}|x-y| \leqslant \rho_{F}(x, y) \leqslant c_{2}|x-y|$, where

$$
\rho_{F}(x, y):=\inf \left\{\int_{0}^{1}|\dot{\gamma}(s)| d s: \gamma \in C^{1}([0,1] ; F), \gamma(0)=x, \gamma(1)=y\right\}
$$

is the intrinsic distance on $F, m$ is the Lebesgue measure, and $B_{F}(x, r)$ is the ball with respect to $\rho_{F}$. For example, these properties are satisfied when $F$ is an inner uniform domain; see [32, Chapter 2.3]. For $x=\left(x_{1}, \cdots, x_{d}\right) \in n^{-1} \mathbb{Z}^{d}$, set $U_{n}(x)=\prod_{i=1}^{d}\left[x_{i}, x_{i}+n^{-1}\right)$. Note that when $F$ is the closure of a bounded Lipschitz domain, $V_{n}:=\left\{n^{-1} \mathbb{Z}^{d} \cap F: U_{n}(x) \subset F\right\}$ satisfies the properties given in Lemma 4.1. Suppose that $\left\{w_{x, y}:(x, y) \in E\right\}$ is a sequence of independent random variables satisfying the conditions in Proposition 5.6. Then the conclusion of Proposition 5.6 holds on F. Indeed, in this case, by taking $V_{n}$ as above, the proofs of Theorem 5.3 and Proposition 5.6 go through without any change (with $\rho$ replaced by $\rho_{F}$ as explained in Remark 5.1). Note that neither $V_{n}=n^{-1} V_{1}$ nor $X_{t}^{(n), \omega}=n^{-1} X_{n^{\alpha} t}^{V_{1}, \omega}$ holds in general in this setting. (However, we can verify that $X_{t}^{(n), \omega}=n^{-1} X_{n^{\alpha} t}^{\tilde{V}_{n}, \omega}$, where $\tilde{V}_{n}:=n V_{n} \subset n F$.) Note that the proofs do not require these properties, and the integrability condition given for all $x, y \in \mathbb{Z}^{d}$ is (more than) enough for the estimates in the proofs to hold.

5.2.4. Fractal graph. The arguments in Example 5.2.1 work for more general graphs that satisfy (i)-(iv), and that its scaling limit $(F, \rho, m)$ and Dirichlet form which satisfy (MMS) and (Dir.) respectively as discussed at the beginning of subsection 5.1. In particular, we can prove quenched invariance principle for stable-like processes on various fractal graphs.

Here we introduce the most typical fractal graph; namely the Sierpinski gasket graph. Let $e_{0}=$ $(0,0, \cdots, 0) \in \mathbb{R}^{N}$, and for $1 \leqslant i \leqslant N, e_{i}$ be the unit vector in $\mathbb{R}^{N}$ whose $i$-th element is 1 . Set $F_{i}(x)=\left(x-e_{i}\right) / 2+e_{i}$ for $0 \leqslant i \leqslant N$. Then, there exists the unique non-void compact set such that $K=\cup_{i=0}^{N} F_{i}(K) ; K$ is called the $N$-dimensional Sierpinski gasket. Set $F:=\cup_{n=0}^{\infty} 2^{n} K$, which is the unbounded Sierpinski gasket. Let

$$
V_{1}=\bigcup_{m=0}^{\infty} 2^{m}\left(\bigcup_{i_{1}, \cdots, i_{m}=0}^{N} F_{i_{1}} \circ \cdots \circ F_{i_{m}}\left(\left\{e_{0}, \cdots, e_{N}\right\}\right)\right), V_{n}=2^{-n+1} V_{1} .
$$

(Hence, $n^{-1}$ in the definition of $V_{n}$ in the previous subsection is now $2^{-n+1}$.) The closure of $\cup_{m \geqslant 1} V_{m}$ is $F . \quad F$ satisfies assumption (MMS) with $d=\log (N+1) / \log 2$. We can naturally construct a regular stable-like Dirichlet form satisfying assumption (Dir.). Let $\left\{w_{x, y}: x, y \in V_{1}\right\}$ be a sequence of independent random variables. Then we have Proposition 5.6 with the same proof in this case as well.

5.2.5. Constant speed $\alpha$-stable-like random walk on $\mathbb{Z}^{d}$. We have considered in Theorem 1.1 the quenched invariance principle for variable speed $\alpha$-stable-like random walks. In this part, we will show the quenched invariance principle for a class of constant speed $\alpha$-stable-like random walks on $\mathbb{Z}^{d}$, by using Theorem 1.1 and the time change argument.

Let $\mathbb{L}=\mathbb{Z}^{d}$ with $d>4-2 \alpha$, and $E$ be the set of all unordered pairs on $\mathbb{Z}^{d}$. Suppose that $\left\{w_{x, y}(\omega):(x, y) \in E\right\}$ is the set of mutually independent non-negative random variables such that $\mathbb{E}\left[w_{x, y}\right]=1,(1.2)$ and (1.3) hold. Suppose for simplicity that they have the same distribution $\lambda$ on $[0, \infty)$. In the following, without loss of generality we take $\Omega=[0, \infty)^{E}, \mathbb{P}=\prod_{(x, y) \in E} \lambda$ and $w_{x, y}(\omega)=\omega(x, y)$ for any $\omega \in \Omega$. For every $z \in \mathbb{Z}^{d}$, define $\tau_{z}: \Omega \rightarrow \Omega$ by $\tau_{z} \omega(x, y)=\omega(x+z, y+z)$. It is standard to verify that $\mathbb{P}$ is stationary and ergodic with respect to the translations $\left\{\tau_{z}\right\}_{z \in \mathbb{Z}^{d}}$ of $\mathbb{Z}^{d}$, see e.g. the proof of [14, Theorem 3.2].

Let $\left(X_{t}^{\omega}\right)_{t \geqslant 0}$ be the $\mathbb{Z}^{d}$-valued process as in Theorem 1.1, which is a variable speed $\alpha$-stable-like random walk. Now we define a constant speed $\alpha$-stable-like random walk $\left(Z_{t}^{\omega}\right)_{t \geqslant 0}$ via the time change as follows

$$
Z_{t}^{\omega}:=X_{a_{t}(\omega)}^{\omega},
$$


where $a_{t}(\omega):=\inf \left\{s \geqslant 0: A_{s}(\omega) \geqslant t\right\}, A_{t}(\omega):=\int_{0}^{t} \nu_{X_{s}^{\omega}}(\omega) d s$ and $\nu_{x}(\omega):=\sum_{y \in \mathbb{Z}^{d}: y \neq x} \frac{\omega(x, y)}{|x-y| d+\alpha}$ for every $x \in \mathbb{Z}^{d}$. Letting $Z_{t}^{(n), \omega}:=n^{-1} Z_{n^{\alpha} t}^{\omega}$ and $X_{t}^{(n), \omega}:=n^{-1} X_{n^{\alpha} t}^{\omega}$, we have $Z_{t}^{(n), \omega}=X_{a_{t}^{(n)}(\omega)}^{(n), \omega}$, where $a_{t}^{(n)}(\omega):=n^{-\alpha} a_{n^{\alpha} t}(\omega)$.

According to the argument of [3, Lemma 2.4], we can prove that $\mathbb{P}$ is stationary, reversible and ergodic with respect to the environment process $\left(\tau_{X_{t}^{\omega} \omega} \omega\right)_{t \geqslant 0}$. Therefore, by the ergodic theorem, we have

$$
\lim _{t \rightarrow \infty} \frac{A_{t}(\omega)}{t}=\lim _{t \rightarrow \infty} \frac{\int_{0}^{t} \nu_{0}\left(\tau_{X_{s} \omega} \omega\right) d s}{t}=\mathbb{E}\left[\nu_{0}(\omega)\right]=\sum_{y \in \mathbb{Z}^{d}: y \neq 0} \frac{1}{|y|^{d+\alpha}}=: C_{d, \alpha}, \quad \text { a.s. }
$$

Combining this with the strictly increasing property of $t \mapsto a_{t}(\omega)$ (which is due to the fact that $\nu_{x}(\omega)>0$ ), we prove that for every $T>0$,

$$
\begin{aligned}
& \lim _{n \rightarrow \infty} \sup _{t \in[0, T]}\left|a_{t}^{(n)}(\omega)-C_{d, \alpha}^{-1} t\right| \\
& =\lim _{n \rightarrow \infty} \sup _{t \in[0, T]}\left|n^{-\alpha} a_{n^{\alpha} t}(\omega)-C_{d, \alpha}^{-1} t\right|=0 \\
& \leqslant \lim _{n \rightarrow \infty} \sup _{t \in\left[n^{-\delta}, T\right]}\left|n^{-\alpha} a_{n^{\alpha} t}(\omega)-C_{d, \alpha}^{-1} t\right|+\lim _{n \rightarrow \infty} \sup _{t \in\left[0, n^{-\delta}\right]}\left(n^{-\alpha} a_{n^{\alpha} t}(\omega)+C_{d, \alpha}^{-1} t\right) \\
& \leqslant \lim _{n \rightarrow \infty} \sup _{t \in\left[n^{-\delta}, T\right]}\left|n^{-\alpha} a_{n^{\alpha} t}(\omega)-C_{d, \alpha}^{-1} t\right|+\lim _{n \rightarrow \infty}\left(n^{-\alpha} a_{n^{\alpha-\delta}}(\omega)+C_{d, \alpha}^{-1} n^{-\delta}\right)=0, \text { a.s., }
\end{aligned}
$$

where $\delta$ is any fixed positive constant such that $\delta<\alpha$.

According to Theorem 1.1, for any $T>0$ and a.s. $\omega \in \Omega, X^{(n), \omega}$ converges under the Skorohod topology on $\mathscr{D}\left([0, T] ; \mathbb{R}^{d}\right)$ to a symmetric $\alpha$-stable Lévy process $Y$. on $\mathbb{R}^{d}$ with jumping measure $|z|^{-d-\alpha} d z$. Then, by the fact that $Z_{t}^{(n), \omega}=X_{a_{t}^{(n)}(\omega)}^{(n), \omega},(5.18)$ and the definition of the Skorohod topology, we can obtain that, for any $T>0$ and a.s. $\omega \in \Omega, Z^{(n), \omega}$ converges under the Skorohod topology on $\mathscr{D}\left([0, T] ; \mathbb{R}^{d}\right)$ to the process $Y_{C_{d, \alpha}^{-1}}$.

Acknowledgements. The authors are very grateful to referees for their helpful suggestions of corrections. The research of Xin Chen is supported by National Natural Science Foundation of China (No. 11501361 and No. 11871338). The research of Takashi Kumagai is supported by the Grant-in-Aid for Scientific Research (A) 25247007 and 17H01093, Japan. The research is supported by the National Natural Science Foundation of China (No. 11831014), the Program for Probability and Statistics: Theory and Application (No. IRTL1704) and the Program for Innovative Research Team in Science and Technology in Fujian Province University (IRTSTFJ).

\section{REFERENCES}

[1] Aldous, D.: Stopping times and tightness, Ann. Probab. 6 (1978) 335-340.

[2] Andres, S., Barlow, M.T., Deuschel, J.-D. and Hambly, B.M.: Invariance principle for the random conductance model, Probab. Theory Relat. Fields 156 (2013) 535-580.

[3] Andres, S., Deuschel, J.-D. and Slowik, M.: Invariance principle for the random conductance model in a degenerate ergodic environment, Ann. Probab. 43 (2015) 1866-1891.

[4] Andres, S., Deuschel, J.-D. and Slowik, M.: Harnack inequalities on weighted graphs and some applications to the random conductance model, Probab. Theory Relat. Fields 166 (2017) 713-752.

[5] Andres, S., Chiarini, A., Deuschel, J.-D. and Slowik, M.: Quenched invariance principle for random walks with time-dependent ergodic degenerate weights, Ann. Probab. 46 (2018) 302-336.

[6] Barlow, M.T.: Random walks on supercritical percolation clusters, Ann. Probab. 32 (2004) 3024-3084.

[7] Barlow, M.T., Bass, R.F. and Kumagai, T.: Parabolic Harnack inequality and heat kernel estimates for random walks with long range jumps, Math. Z. 261 (2009) 297-320.

[8] Barlow, M.T. and Chen, X.X.: Gaussian bounds and parabolic Harnack inequality on locally irregular graphs, Math. Ann. 366 (2016) 1677-1720.

[9] Barlow, M.T. and Deuschel, J.-D.: Invariance principle for the random conductance model with unbounded conductances, Ann. Probab. 38 (2010) 234-276.

[10] Barlow, M.T., Grigor'yan, A. and Kumagai, T.: Heat kernel upper bounds for jump processes and the first exit time, J. Reine Angew. Math. 626 (2009) 135-157.

[11] Barlow, M.T. and Hambly. B.M.: Parabolic Harnack inequality and local limit theorem for random walks on percolation clusters, Electron. J. Probab. 14 (2009) 1-27. 
[12] Bass, R.F.: On Aronsen's upper bounds for heat kernels, Bull. London Math. Soc. 34 (2002) 415-419.

[13] Berger, N.: Transience, recurrence and critical behavior for long-range percolation, Commun. Math. Phys. 226 (2002) 531-558.

[14] Berger, N. and Biskup, M.: Quenched invariance principle for simple random walk on percolation clusters, Probab. Theory Relat. Fields 137 (2007) 83-120.

[15] Biskup, M.: On the scaling of the chemical distance in long range percolation models, Ann. Probab. 32 (2004) 2938-2977.

[16] Biskup, M.: Recent progress on the random conductance model, Probab. Surv. 8 (2011) 294-373.

[17] Biskup, M and Prescott, T.-M.: Functional CLT for random walk among bounded random conductances, Electron. J. Probab. 12 (2007) no. 49, 1323-1348.

[18] Biskup, M. and Rodriguez, P.-F.: Limit theory for random walks in degenerate time-dependent random environments, J. Funct. Anal. 274 (2018) 985-1046.

[19] Chen, X., Chen, Z.-Q., Kumagai, T. and Wang, J.: Quenched invariance principle for long range random walks in balanced random environments, preprint 2019.

[20] Chen, X., Kumagai, T. and Wang, J.: Random conductance models with stable-like jumps: heat kernel estimates and Harnack inequalities, available at arXiv: 1808.02178.

[21] Chen, Z.-Q., Croydon, D.A. and Kumagai, T.: Quenched invariance principles for random walks and elliptic diffusions in random media with boundary, Ann. Probab. 43 (2015) 1594-1642.

[22] Chen, Z.-Q., Kim, P. and Kumagai, T.: Weighted Poincaré inequality and heat kernel estimates for finite range jump processes, Math. Ann. 342 (2008) 833-883.

[23] Chen, Z.-Q., Kim, P. and Kumagai, T.: Discrete approximation of symmetric jump processes on metric measure spaces, Probab. Theory Relat. Fields 155 (2013) 703-749.

[24] Chen, Z.-Q. and Kumagai, T.: Heat kernel estimates for stable-like processes on $d$-sets, Stoch. Proc. Appl. 108 (2003) 27-62.

[25] Chen, Z.-Q. and Kumagai, T.: Heat kernel estimates for jump processes of mixed types on metric measure spaces, Probab. Theory Relat. Fields 140 (2008) 277-317.

[26] Chen, Z.-Q., Kumagai, T. and Wang, J.: Stability of heat kernel estimates for symmetric non-local Dirichlet forms, to appear in Memoirs of the AMS, available at arXiv:1604.04035.

[27] Crawford, N. and Sly, A.: Simple random walks on long range percolation cluster I: heat kernel bounds, Probab. Theory Relat. Fields 154 (2012) 753-786.

[28] Crawford, N. and Sly, A.: Simple random walks on long range percolation cluster II: scaling limits, Ann. Probab. 41 (2013) 445-502.

[29] Davies, E.B.: Large deviations for the heat kernels on graphs, J. London Math. Soc. 23 (1993) 65-72.

[30] Deuschel, J., Nguyen, T. and Slowik, M.: Quenched invariance principles for the random conductance model on a random graph with degenerate ergodic weights, Probab. Theory Relat. Fields 170 (2018) 363-386.

[31] Ethier, S.N. and Kurtz, T.G.: Markov Processes: Characterization and Convergence, John Wiley \& Sons, New York, 1986.

[32] Gyrya, P. and Saloff-Coste, L.: Neumann and Dirichlet heat kernels in inner uniform domains, Astérisque 336 (2011) viii+144 pp.

[33] Husseini, R. and Kassmann, M.: Markov chain approximations for symmetric jump processes, Potential Anal. 27 (2007) 353-380.

[34] Kumagai, T.: Random walks on disordered media and their scaling limits, Lect. Notes in Math. 2101, Ecole d'été de probabilités de Saint-Flour XL-2010, Springer, New York 2014.

[35] Mathieu, P.: Quenched invariance principles for random walks with random conductances, J. Stat. Phys. 130 (2008) 1025-1046.

[36] Mathieu, P. and Piatnitski, A.: Quenched invariance principles for random walks on percolation clusters, Proc. Roy. Soc. A 463 (2007) 2287-2307.

[37] Nash, J.: Continuity of solutions of parabolic and elliptic equations, Amer. J. Math. 80 (1958) 931-954.

[38] Procaccia, E., Rosenthal, R. and Sapozhnikov, A.: Quenched invariance principle for simple random walk on clusters in correlated percolation models, Probab. Theory Relat. Fields 166 (2016) 619-657.

[39] Sidoravicius, V. and Sznitman, A.-S.: Quenched invariance principles for walks on clusters of percolation or among random conductances, Probab. Theory Relat. Fields 129 (2004) 219-244. 